\title{
MULTIDISCIPLINARY DESIGN OPTIMIZATION OF AN EXTREME ASPECT RATIO HALE UAV
}

\author{
A Thesis \\ presented to \\ the Faculty of the California Polytechnic State University, \\ San Luis Obispo
}

In Partial Fulfillment

of the Requirements for the Degree

Master of Science in Aerospace Engineering

by

Bryan J. Morrisey

June 2009 
(C) 2009

Bryan J. Morrisey

ALL RIGHTS RESERVED

ii 


\section{COMMITTEE MEMBERSHIP}

Title:

Author:

Date Submitted:
Multidisciplinary Design

Optimization of an Extreme Aspect

Ratio HALE UAV

Bryan J. Morrisey

June 2009

Committee Chair: $\quad$ Dr. Robert A. McDonald

Committee Member: $\quad$ Dr. David D. Marshall

Committee Member: Dr. Tim Takahashi

Committee Member: Dr. John Dunning 


\begin{abstract}
Multidisciplinary Design Optimization of an Extreme Aspect Ratio HALE UAV

Bryan J. Morrisey
\end{abstract}

Development of High Altitude Long Endurance (HALE) aircraft systems is part of a vision for a low cost communications/surveillance capability. Applications of a multi payload aircraft operating for extended periods at stratospheric altitudes span military and civil genres and support battlefield operations, communications, atmospheric or agricultural monitoring, surveillance, and other disciplines that may currently require satellite-based infrastructure. Presently, several development efforts are underway in this field, including a project sponsored by DARPA that aims at producing an aircraft that can sustain flight for multiple years and act as a pseudo-satellite. Design of this type of air vehicle represents a substantial challenge because of the vast number of engineering disciplines required for analysis, and its residence at the frontier of energy technology.

The central goal of this research was the development of a multidisciplinary tool for analysis, design, and optimization of HALE UAVs, facilitating the study of a novel configuration concept. Applying design ideas stemming from a unique WWII-era project, a "pinned wing" HALE aircraft would employ self-supporting wing segments assembled into one overall flying wing. The research effort began with the creation of a multidisciplinary analysis environment comprised of analysis modules, each providing information about a specific discipline. As the modules were created, attempts were made to validate and calibrate the processes against known data, culminating in a validation 
study of the fully integrated MDA environment. Using the NASA / AeroVironment Helios aircraft as a basis for comparison, the included MDA environment sized a vehicle to within $5 \%$ of the actual maximum gross weight for generalized Helios payload and mission data. When wrapped in an optimization routine, the same integrated design environment shows potential for a $17.3 \%$ reduction in weight when wing thickness to chord ratio, aspect ratio, wing loading, and power to weight ratio are included as optimizer-controlled design variables.

Investigation of applying the sustained day/night mission requirement and improved technology factors to the design shows that there are potential benefits associated with a segmented or pinned wing. As expected, wing structural weight is reduced, but benefits diminish as higher numbers of wing segments are considered. For an aircraft consisting of six wing segments, a maximum of $14.2 \%$ reduction in gross weight over an advanced technology optimal baseline is predicted. 


\section{ACKNOWLEDGEMENTS}

I am greatly indebted to many of my friends, peers, and mentors for all of their support through my career at Cal Poly. Their help has allowed me to keep a balanced and exciting life despite long hours spent at school and in the lab.

I was lucky enough to meet Dr. McDonald early in both his and my time at the university. His enthusiasm for all things aircraft and seemingly unlimited databases of aircraft information and stories kept the education fresh and interesting. Over the past several years I have been employed by the Aerospace Department under both Dr. McDonald and Dr. Marshall, allowing me to exercise my knowledge while paying my rent, and I thank you both for that. Dr. Takahashi has provided valuable insight into several of the methods and processes contained herein, something greatly appreciated considering the hectic schedules and travel time required. Also I would like to thank Dr. Dunning for his enthusiasm with this project, as well as the industry connections he facilitated. Lastly, to all the professors, instructors, and mentors of my past, thank you so much for all of your hard work. My experience teaching the sophomores here has given me a new perspective towards the work you do; it is truly a service to the nation.

Now, to all of my friends and roommates over the years, I owe you an enormous debt of gratitude. To the aero folk, the amount of sleep lost and stress imposed on our lives would break any lesser student, and I am glad that during the hardest times I always had a bunch of friends to fall back on, commiserate with, or study with. Doing this alone would be impossible. For the non aero friends, let's just say I don't want to think about how I'd have turned out if it weren't for you all. Thanks for being there when I needed a 
night off from school, I'm sure I still owe many of you a beer, so don't be shy about cashing in. David and Ryan, you guys rock. Putting up with all of my shenanigans must have been trying at times, not to mention the weeks where we wouldn't get to hang out because of school. I hope to see you often in the future, and keep the great times rolling. Finally, I cannot go without a strong nod to Human League, the men, the myth, the legend, and my senior design team. You all gave me confidence that among our engineering brethren, there are others who value having fun as much as doing good work, and are capable of both.

In addition to my friends and colleagues, I must thank my family for everything they have sacrificed to see me through to this point. Mom and Dad, what can I say except that I love you and could not have asked for anything more. I would consider myself lucky to one day have a family as strong as the one you have built, but I do not doubt that you have given me the tools to do so. Danielle, it is fun to watch you grow up a couple years behind me, and I am very excited to see where your life will lead you. You definitely have great things ahead. To Nonna, Poppop, and all the rest of my family, it is unfortunate that we are so geographically separated, but it makes me appreciate the times we are together just that much more. I love you all, and thank you for everything. 


\section{TABLE OF CONTENTS}

LIST OF TABLES ........................................................................................................... xi

LIST OF FIGURES ............................................................................................... xiii

NOMENCLATURE.................................................................................................... $\mathrm{xvii}$

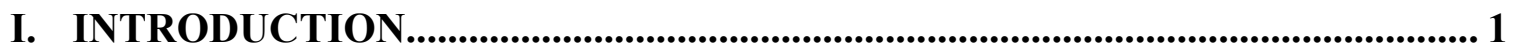

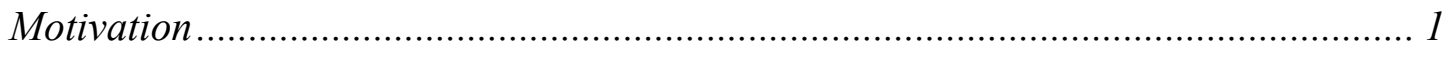

Electric Aircraft Background.................................................................... 4

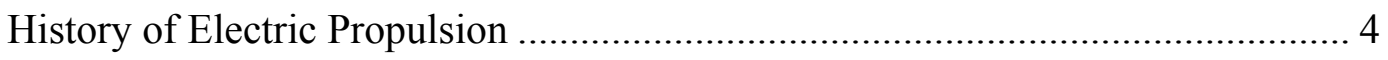

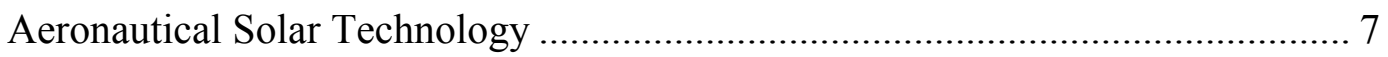

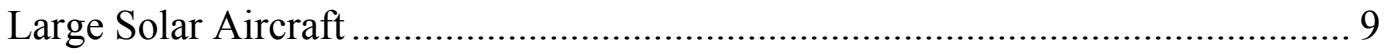

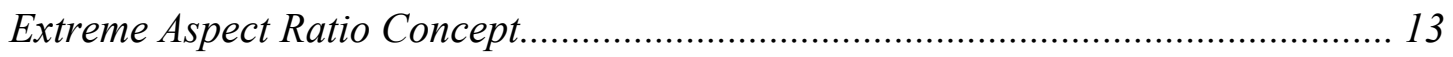

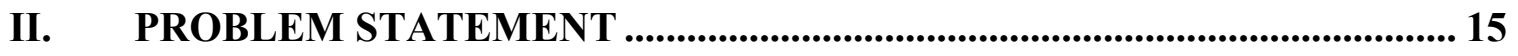

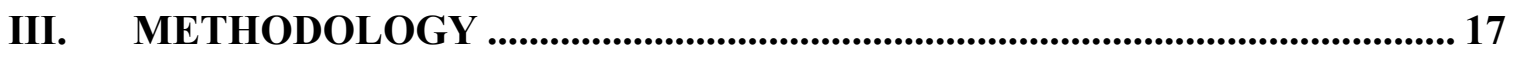

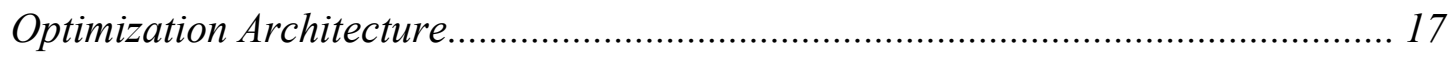

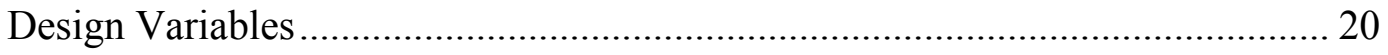

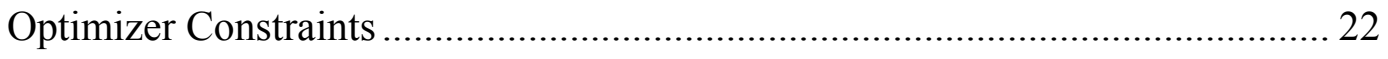

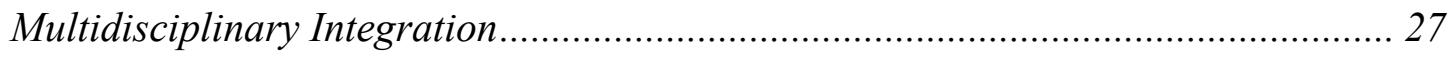

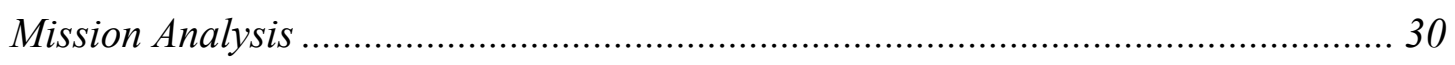

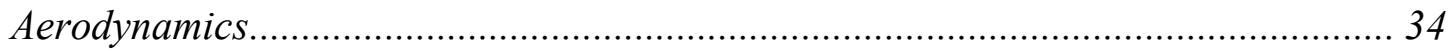

Viii 
Induced Drag

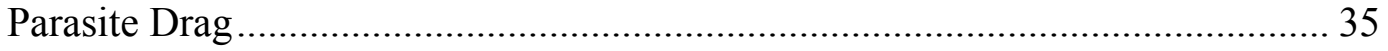

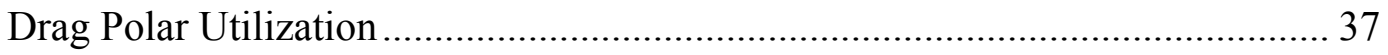

Drag Polar Validation ............................................................................ 43

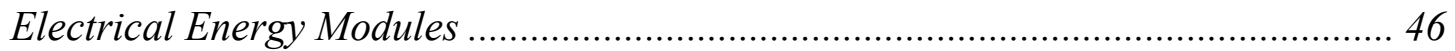

Solar Energy Generation.......................................................................... 46

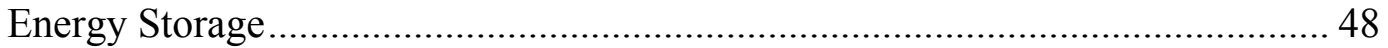

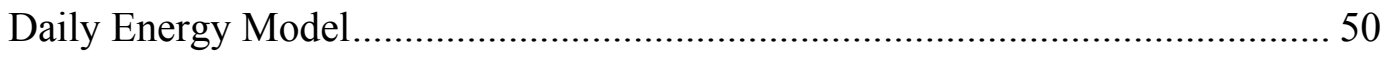

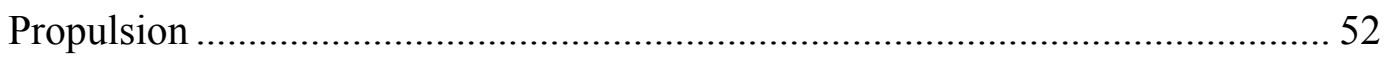

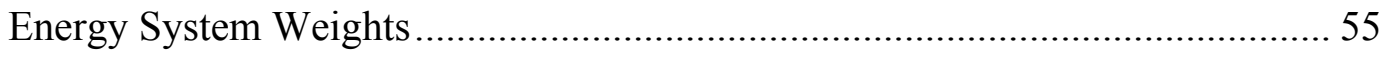

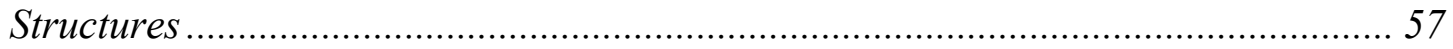

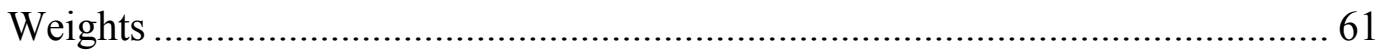

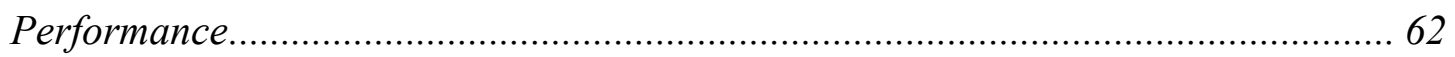

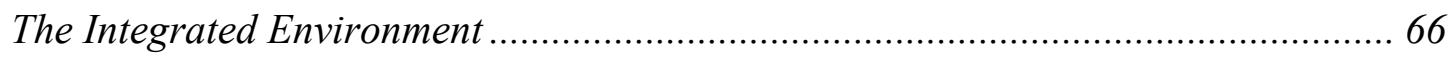

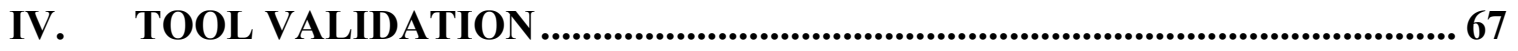

Maximum Lift Coefficient Response ................................................................ 71

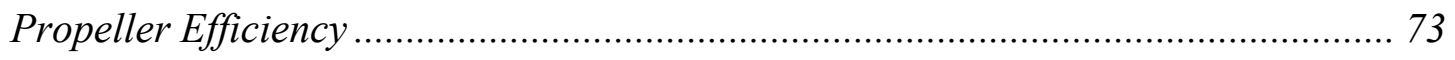

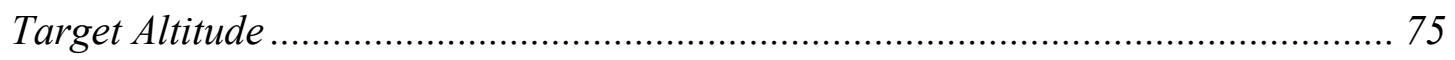

Night Flight and Battery Requirement ........................................................ 76 
Stage I: Power and Wing Loading as Design Variables ......................................... 81

Stage II: Adding Aspect Ratio as a Design Variable ................................................ 84

Stage III a: Full Optimization of "Daytime Plus" Mission ....................................... 87

Stage III b: Four Variable Optimization of Long Endurance Baseline...................... 92

Stage IV: Full Optimization of Long Endurance Baseline ....................................... 95

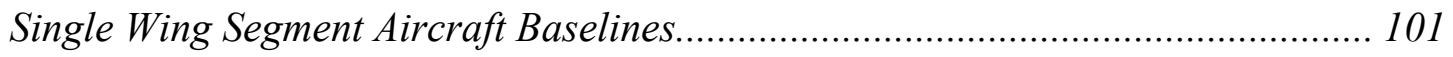

Different Payloads: Weight and Power Requirement............................................ 104

VI. SEGMENTED WING OPTIMIZATION ...................................................... 107

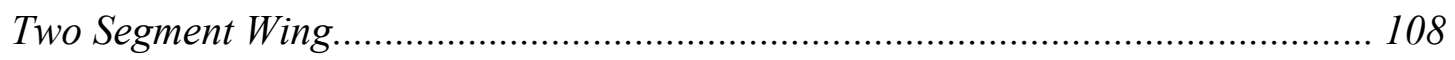

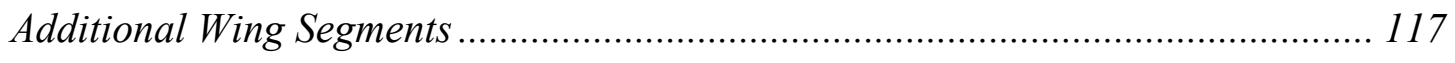

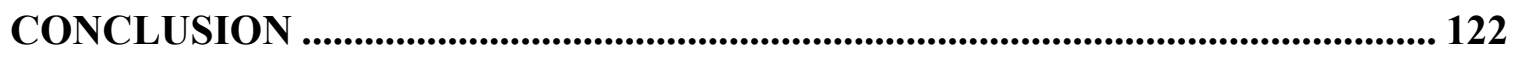

FUTURE WORK ........................................................................................................... 125

REFERENCES................................................................................................................ 127 


\section{LIST OF TABLES}

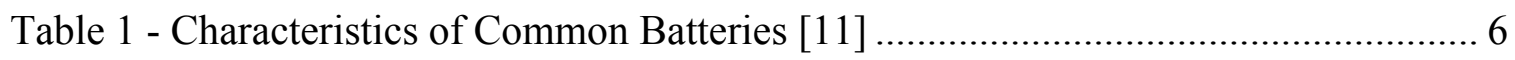

Table 2 - Initial Optimizer Design Variables ................................................................ 20

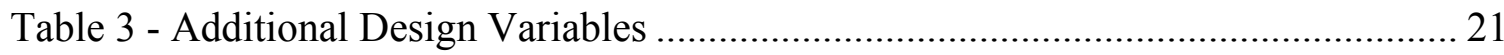

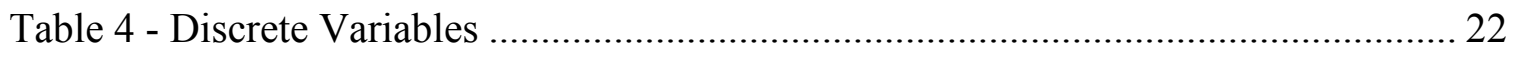

Table 5 - Fundamental Design Constraints............................................................... 25

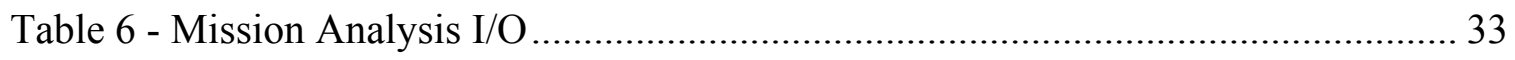

Table 7 - General Power Train Efficiencies.................................................................. 53

Table 8 - Specific Power Train Efficiencies [36] ……………………....................... 53

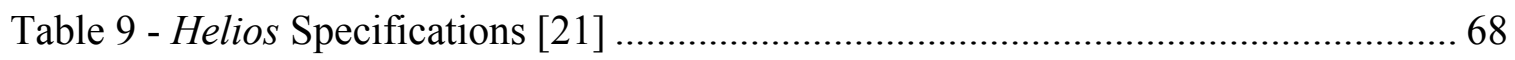

Table 10 - Configuration Variables for Validation Case ................................................... 68

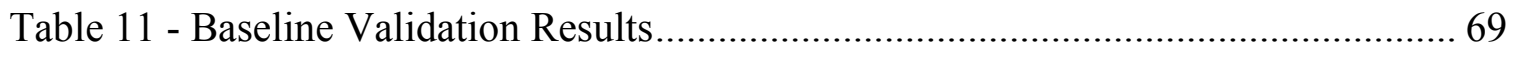

Table 12 - Stage I Baseline Optimization Summary ……………………………....... 82

Table 13 - Stage II Optimization Summary ………………......................................... 84

Table 14 - Stage III(a) Optimization Summary ……………………............................ 88

Table 15 - Optimization Data Summary ………………………................................. 91

Table 16 - Parameters Defining the Long Endurance Baseline .......................................... 93

Table 17 - Stage III(b) Optimization Summary ............................................................ 93

Table 18 - Stage IV Optimization Summary ……………………................................. 95

Table 19 - Focused Start Point Optimization Summary ................................................... 98

Table 20 - Baseline Aircraft Summary ………………….......................................... 103

Table 21 - Payload Study Optima Comparison …………………………................... 106 
Table 22 - Two Segment Wing Optimization Summary........................................ 108

Table 23 - Average Values for Solution Clusters .................................................. 115 


\section{LIST OF FIGURES}

Figure 1 - Fred Militky's Silentius (left) and Hi-Fly (right) [10] .................................. 4

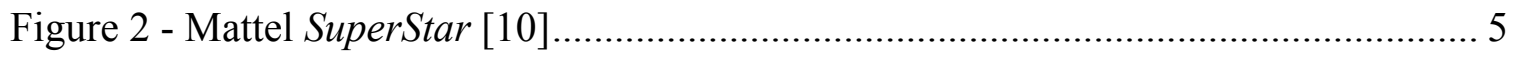

Figure 3 - Sunrise II Flight Preparation [9] ............................................................. 7

Figure 4 - Solong Multi Day Solar Aircraft (http://machinedesign.com) ........................ 8

Figure 5 - The Gossamer Penguin in Flight [9] ...................................................... 9

Figure 6 - Solar Challenger Flying Over the English Channel .................................... 10

Figure 7 - Solar Aircraft in the ERAST Program [20]............................................... 11

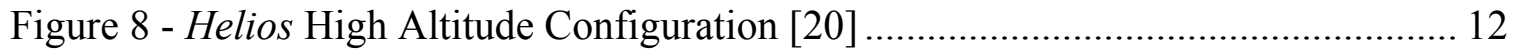

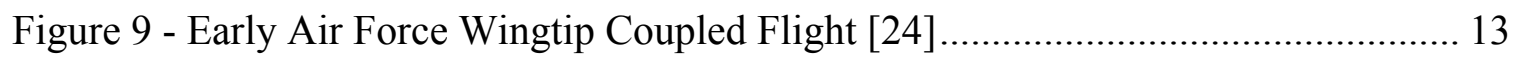

Figure 10 - Extreme Aspect Ratio Concept .............................................................. 14

Figure 11 - HALE UAV Mission Profile ................................................................. 15

Figure 12 - Theoretical Aircraft Design Structure Matrix ........................................... 19

Figure 13 - Classical Aircraft Sizing Process ............................................................ 27

Figure 14 - Multidisciplinary Design Analysis and Optimization Architecture.............. 30

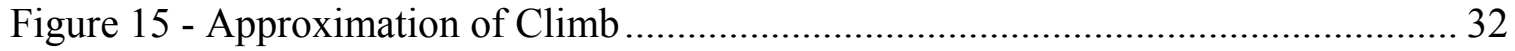

Figure 16 - Comparison of Mission Analysis Methods ............................................... 33

Figure 17 - General Wing Modeled in AVL With Loading Distribution ......................... 34

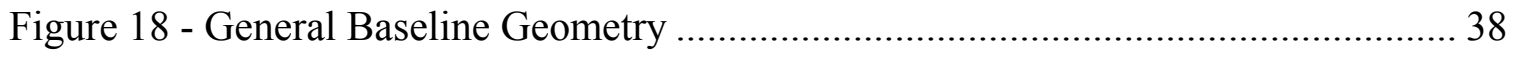

Figure 19 - Drag Polars During the Helios Baseline Analysis ...................................... 39

Figure 20 - Reynolds' Number Based on Chord .......................................................... 40

Figure 21 - Parasite Drag Breakdown, Component Drag Counts.................................. 40

xiii 
Figure 22 - Effect of Aspect Ratio on Drag ................................................................... 41

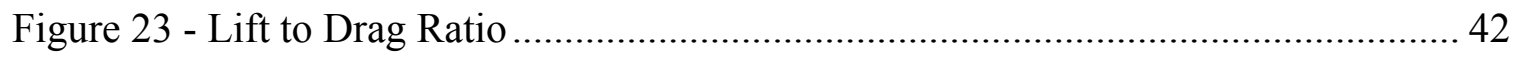

Figure 24 - GlobalFlyer Modeled in VSP for Drag Validation......................................... 43

Figure 25 - Cruise Drag Polar for GlobalFlyer .......................................................... 44

Figure 26 - Parasite Drag Buildup for GlobalFlyer, Component Drag Counts................ 44

Figure 27 - Effect of Wing Thickness on Drag.............................................................. 45

Figure 28 - Basis for Available Solar Energy Models [14], [36], [37]........................... 48

Figure 29 - Model for Available and Required Power (above) and Resulting Extra Power

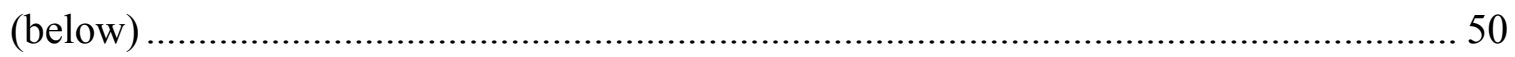

Figure 30 - Energy Storage Charge Profile .................................................................. 51

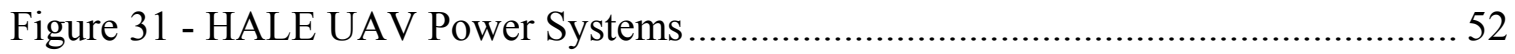

Figure 32 - Helios Wing Structural Arrangement [50]............................................... 58

Figure 33 - Structural Analysis Process................................................................. 58

Figure 34 - General Spanwise Loading Distribution ........................................................ 59

Figure 35 - General Spanwise Shear and Moment Diagrams........................................... 59

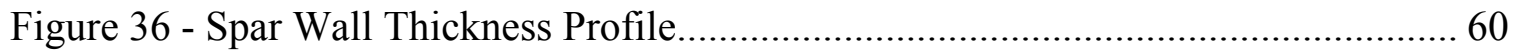

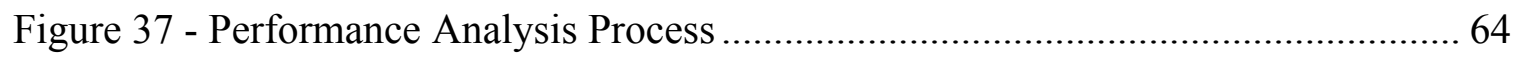

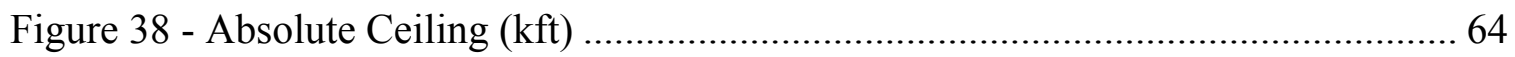

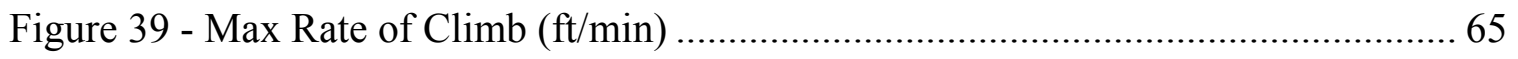

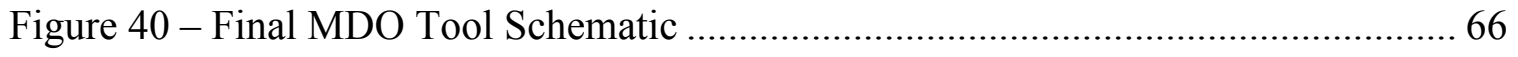

Figure 41 - Baseline Weight Breakdown...................................................................... 70

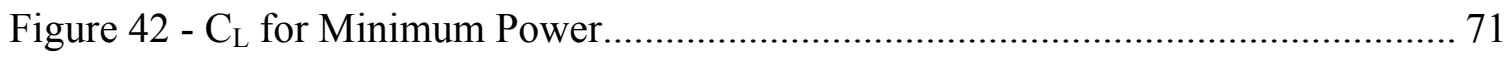

Figure 43 - Response to $C_{\mathrm{Lmax}}$ Perturbation.................................................................... 72 


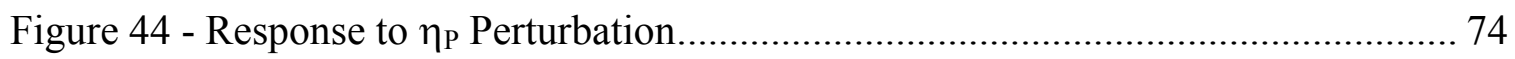

Figure 45 - Response to Target Altitude Perturbation.................................................. 76

Figure 46 - Response to Night Flight Requirement..................................................... 78

Figure 47 - Response to Improved Battery Technology …………………………….... 78

Figure 48 - Optimal Baseline Development Procedure ................................................. 80

Figure 49 - Scaled Design Variable History ………………........................................ 82

Figure 50 - Stage I Objective Function History ........................................................... 83

Figure 51 - Stage I Optimization Component Weights ................................................ 84

Figure 52 - Stage II Scaled Design Variable History ………………………………..... 85

Figure 53 - Stage II Objective Function History ......................................................... 86

Figure 54 - Comparison of Optimal Weight Breakdowns ................................................ 86

Figure 55 - Maximum Constraint Violation History ......................................................... 87

Figure 56 - Stage III(a) Design Variable History ……………………........................ 88

Figure 57 - Stage III(a) Objective Value History ……………….................................. 89

Figure 58 - Progression of Optimal Weight Breakdowns............................................... 89

Figure 59 - Spar Design for Two Configurations ………………………………......... 90

Figure 60 - Weight Breakdown for the ATLEB Compared to Earlier Results.................. 94

Figure 61 - Stage IV Design Variable History.................................................................. 96

Figure 62 - Comparison of Stage IV Weight to Previous Optima....................................... 97

Figure 63 - Weight Breakdown for Revised Stage IV Optimization................................. 98

Figure 64 - Weight Comparison for Progressive Baseline Optimal Configurations ........ 99

Figure 65 - Desired Battery Charge Profile …………………………………………. 100

Figure 66 - Helios Proxy Alongside Two Optimal Baselines........................................... 101 
Figure 67 - Comparison of Optimized Baseline Aircraft to NASA ERAST Family...... 102

Figure 68 - Weight Breakdown for Different Payload Weights ................................... 104

Figure 69 - Battery, Solar, and Wing Weight Sensitivity to Payload ........................... 105

Figure 70 - Weight Breakdown for Different Payload Power ................................... 105

Figure 71 - Two Segment Objective Function History .......................................... 109

Figure 72 - Weight Comparison for Two Segment Aircraft..................................... 110

Figure 73 - Plot Matrix of All Optimization Cases for a Two Segment Platform.......... 111

Figure 74 - Two Segment MDO Solution Space .................................................. 112

Figure 75 - Two Segment Solutions Sorted by Gross Weight ................................... 113

Figure 76 - Design Variable History for Anomalous Solutions ................................. 114

Figure 77 - Design Variable History for Attractive Solutions .................................... 115

Figure 78 - Two Segment Solutions: Reynolds' Number ........................................ 116

Figure 79 - Weight Breakdown Comparison of Multiple Wing Segment Configs ........ 117

Figure 80 - Optimal Wing Thickness for Multiple Wing Segments........................... 118

Figure 81 - Optimal Wing Chord for Multiple Wing Segments ................................. 119

Figure 82 - Optimal $\mathrm{S}_{\text {ref }}$ for Multiple Wing Segments........................................... 119

Figure 83 - Optimal Solar Coverage (Percent) .................................................... 120

Figure 84 - Optimal Gross Weight for Multiple Wing Segments............................... 120

Figure 85 - Optimal Wing Loading for Multiple Wing Segments.............................. 121

Figure 86 - Optimal Aspect Ratio for Multiple Wing Segments ............................... 121 


\section{NOMENCLATURE}

\begin{tabular}{|c|c|c|}
\hline A & $=$ & area \\
\hline AR & $=$ & aspect ratio \\
\hline \multirow[t]{2}{*}{ ATLEB } & $=$ & Advanced Technology Long \\
\hline & & Endurance Baseline \\
\hline $\mathrm{b}$ & $=$ & wing span \\
\hline BFGS & $=$ & Broyden-Fletcher-Goldfarb-Shanno \\
\hline $\mathrm{c}$ & $=$ & optimizer inequality constraints \\
\hline $\mathrm{cc}$ & $=$ & cubic centimeter \\
\hline $\mathrm{C}_{\mathrm{D}}$ & $=$ & drag coefficient \\
\hline ceq & $=$ & optimizer equality constraints \\
\hline $\mathrm{C}_{\mathrm{L}}$ & $=$ & finite wing lift coefficient \\
\hline DSM & $=$ & Design Structure Matrix \\
\hline $\mathrm{E}$ & $=$ & energy \\
\hline $\mathrm{e}$ & $=$ & span efficiency factor, Oswald's \\
\hline \multirow{3}{*}{ EDET } & & efficiency \\
\hline & $=$ & Empirical Drag Estimation \\
\hline & & Technique \\
\hline $\mathrm{f}$ & $=$ & optimizer objective function \\
\hline F.F. & $=$ & form factor \\
\hline fc & $=$ & fuel cell \\
\hline $\mathrm{ft}$ & $=$ & foot \\
\hline
\end{tabular}

xvii 
h

HALE

$\mathrm{hr}$

I

$\mathrm{I} / \mathrm{O}$

in

$\mathrm{kg}$

KT

L

L/D

$\mathrm{lb}$

Li-Ion

Li-Po

Li-S

MDA

MDAO

MDO

mo

$\mathrm{N}$

n

$\mathrm{NiCd}$
$=$

$$
=
$$

$=$

$=$

$=$

$=$

$=$

$=$

$=$

$=$

$=$

$=$

$=$

$=$

$=$

$=$

$=$

$=$

$=$

$=$

$=$ altitude

High Altitude Long Endurance

hour

solar irradiance, current

input-output

inch

kilogram

Kuhn-Tucker

liter

lift to drag ratio

lower bound, pound force

Lithium Ion

Lithium Polymer

Lithium-Sulfur

Multidisciplinary Analysis

Multidisciplinary Analysis and

Optimization

Multidisciplinary Design

Optimization

months

number of components

rotational speed, component index

Nickel-Cadmium

xviii 


\begin{tabular}{|c|c|c|}
\hline $\mathrm{Ni}-\mathrm{MH}$ & $=$ & Nickel metal-hydride \\
\hline OBD & $=$ & Optimizer Based Decomposition \\
\hline $\mathrm{P}$ & $=$ & power \\
\hline PEM & $=$ & Proton Exchange Membrane fuel cell \\
\hline \multirow[t]{2}{*}{ POBD } & $=$ & Partial Optimizer Based \\
\hline & & Decomposition \\
\hline PoW & $=$ & power to weight ratio \\
\hline \multirow[t]{2}{*}{ PTMEB } & $=$ & Proven Technology Medium \\
\hline & & Endurance Baseline \\
\hline Q & $=$ & torque \\
\hline $\operatorname{Re}$ & $=$ & Reynolds' number \\
\hline S & $=$ & area \\
\hline SQP & $=$ & Sequential Quadratic Programming \\
\hline $\mathrm{S}_{\mathrm{REF}}, \mathrm{S}_{\mathrm{ref}}$ & $=$ & wing reference area \\
\hline $\mathrm{S}_{\mathrm{WET}}, \mathrm{S}_{\mathrm{wet}}$ & $=$ & wetted area \\
\hline $\mathrm{T}$ & $=$ & thrust \\
\hline $\mathrm{t}$ & $=$ & time \\
\hline$t / c$ & $=$ & wing thickness to chord ratio \\
\hline UAV & $=$ & Unmanned Aerial Vehicle \\
\hline $\mathrm{ub}$ & $=$ & upper bound \\
\hline $\mathrm{V}$ & $=$ & Volt, Velocity \\
\hline W & $=$ & Watt, Weight (lbs) \\
\hline WoS & $=$ & wing loading \\
\hline
\end{tabular}


$\mathrm{X}$

XAR

$\Delta$

$\rho$

$\eta$

$\kappa_{\text {sol_coverage }}$

$\kappa_{\text {watt }}$

Subscripts

Asol

BATT

batt_req

F

FC, fc

G

i

INC

M

o

$\mathrm{P}$

PMD

$$
=
$$$$
=
$$$$
=
$$$$
=
$$$$
=
$$$$
=
$$$$
=
$$

solar cells

power conversion factor area density of solar system

battery

required to be stored in the battery

flat plate friction drag

fuel cell

gearbox

induced drag contribution

incompressible

motor

parasite drag contribution

propeller

power management and distribution 


$\begin{array}{lll}\text { R } & = & \text { required energy at propeller } \\ \text { ref } & = & \text { reference } \\ \text { S } & \text { shaft torque, stored energy, specific } \\ & = & \text { excess power } \\ \text { SC } & = & \text { speed controller } \\ \text { sls } & = & \text { sea level static } \\ \text { sol } & = & \text { solar } \\ \text { SRC } & = & \text { source (batteries, fuel cell, etc.) } \\ \text { TO, to } & = & \text { takeoff } \\ \text { tot } & & \text { total }\end{array}$




\section{INTRODUCTION}

\section{Motivation}

Development of High Altitude Long Endurance (HALE) aircraft systems has long been part of a vision for a low cost communications/surveillance capability [1], [2], [3]. Applications of a multi payload aircraft operating for extended periods at stratospheric altitudes span military and civil genres and support tactical battlefield operations, communications, atmospheric monitoring, precise agricultural and wildfire monitoring, surveillance, and other disciplines requiring satellite-based infrastructure or high resolution imagery [4]. Currently, the Defense Advanced Research Projects Agency (DARPA) is requesting proposals for an aircraft that can sustain flight for multiple years and act as a pseudo-satellite for intelligence, surveillance, and reconnaissance missions [5]. Design of this and any type of air vehicle represents a substantial challenge because of the vast number of engineering disciplines required for analysis. In addition, some tools and analysis methods used in the design of aircraft with more conventional missions may not be applicable to certain types of HALE vehicles. In the modern competitive environment surrounding the manufacture of aircraft systems, oftentimes simply meeting the customer's requirements may not win a contract. Instead, the proposed system must also represent the optimum vehicle for the customer needs [6]. This focus on finding an optimal solution places some additional requirements on the design process itself.

Searching for an overall optimal solution involves broadening the trade space and allowing a large number of variables. These high degree of freedom environments are not handled well by a sequential design process [7]. Also, with highly multivariate 
design spaces, analyzing the sensitivities to each variable individually and relating this information to a whole system sensitivity is a daunting task. One method for mitigating many of the challenges associated with designing complex aeronautical systems is to compile the individual disciplines and analysis methods into one environment, allowing for better organization of data flow, and more efficient communication. This may be accomplished on a small scale by simply bringing codes together on one machine, or in a larger sense by allowing physically separated flight science groups to wrap their analyses for remote use. Once assembled, multidisciplinary analysis, design, and optimization techniques can be applied in the hopes of allowing more broad design spaces and providing a clearer view of system drivers and sensitivities.

With an integrated HALE design environment in place, it is possible to perform parametric studies to investigate areas of potential improvement over current concepts. In essence, we are looking for the active constraints on the design, or the design drivers. Wing design and propulsion systems are the two main aspects of HALE vehicles that are driven by mission requirements, and consequently present the greatest opportunity for system improvements. Accordingly, when considering new or revolutionary design concepts, these two areas should be of primary focus. Generally, HALE aircraft of the past and present, like the U2, Helios, or Global Hawk exhibit high aspect ratio wings that allow the aircraft to achieve the altitudes of interest. Sacrifices are made, however, because with a high aspect ratio planform comes high wing bending moments, unfavorable dynamic structural responses, and large deflections. In the propulsion arena, many current designs feature distributed propulsion, advanced propeller design, and a strong coupling between propulsion and flight controls. Closely related to propulsion is 
the energy source for the aircraft. Most modern internal combustion architectures cannot satisfy the persistent operation requirements of current HALE missions like Vulture or the Communications Relay posed by the AIAA in 2007 that stipulate months to years of continuous flight [8]. As a result, much effort has been devoted to development of environmental energy collection, high energy density storage devices, and other alternative energy concepts [5].

The intent of this research is to evaluate revolutionary changes to HALE aircraft architecture, using state of the art propulsion and energy concepts while breaking new ground for wing design. Wing aspect ratio is the primary characteristic of wing design for the purposes of this study, and the NASA / AeroVironment Helios aircraft set the current threshold for demonstrated all-electric flight with an aspect ratio of 31 . With a new wing concept, it may be possible to push this envelope of high aspect ratio platforms, while simultaneously mitigating problems associated with highly flexible aircraft structures. An integration of architecture-independent design codes into an optimization environment enables identification of constraints that emerge when exploring extreme-aspect-ratio concepts. These constraints take the form of structural and energy requirements such as max stress or minimum specific energy storage density, as well as mission operation requirements that take into account things like available runways and hangers for aircraft with extremely long wingspan. One goal of this paper is to find the area of diminishing returns for wing aspect ratio is such behavior exists, and discuss why and how certain constraints become active. In addition, the work diverges from combustion-based sizing methods and focuses on generalizing the design process for energy-optimized systems and all-electric aircraft. 


\section{Electric Aircraft Background}

\section{History of Electric Propulsion}

Electric propulsion systems in aircraft date back to the 1950's when model aircraft enthusiasts and hobbyists first became successful in the field. The first officially recorded electrically powered flight occurred in June of 1957 with Colonel H.J. Taplin's retro-fitted Radio Queen. Weighing in at $8 \mathrm{lbs}$, the radio controlled model utilized silver/zinc battery cells and a government surplus electric motor in lieu of the stock $3.5 \mathrm{cc}$ diesel engine [9]. Several years later, a coreless motor with an integrated gearbox initially developed for remote control cameras would be adapted to model aircraft. Called the Micromax, this motor was at the heart of the first model developed by Fred Militky for the public, the Silentius [10]. Militky continued development of electric aircraft with the Hi-Fly, working towards the goal of manned electric flight. Figure 1 shows both aircraft.

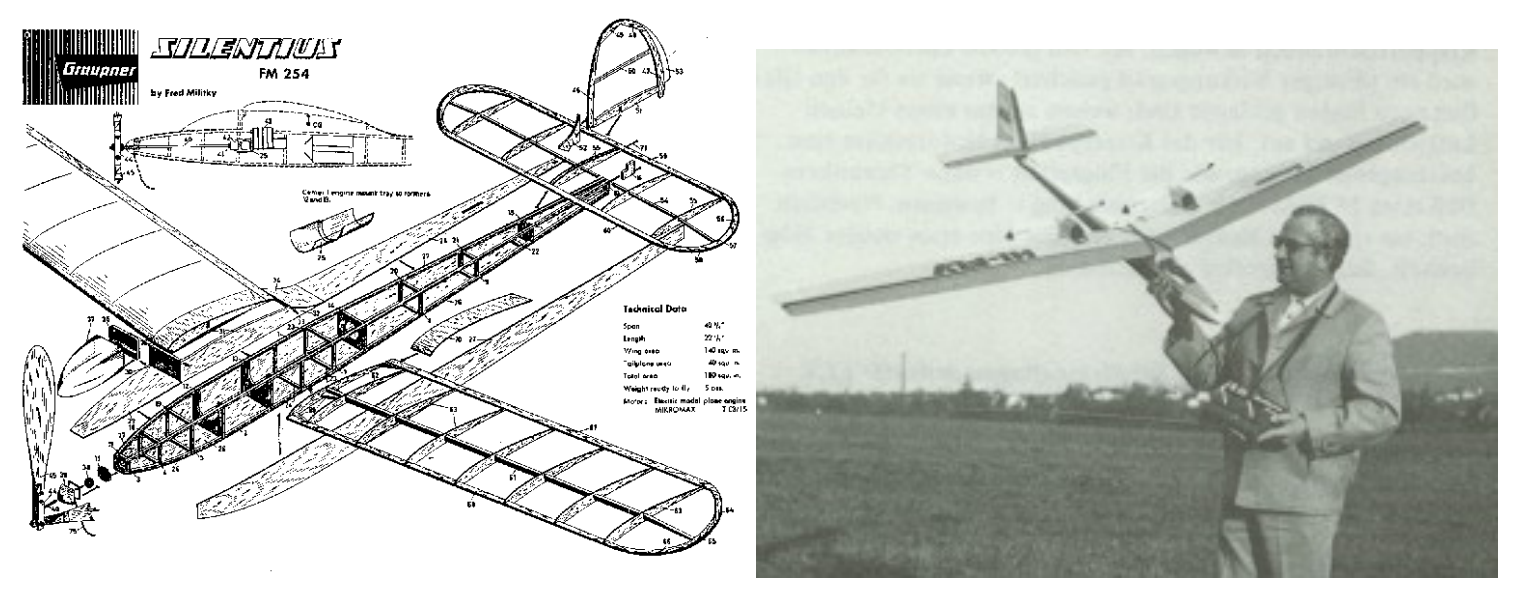

Figure 1 - Fred Militky's Silentius (left) and Hi-Fly (right) [10] 
Later, in the early 1970 's, the first widely commercially available electric model was introduced. Dubbed the Super Star, this aircraft was rechargeable and included a rudder as its only flight control [10].

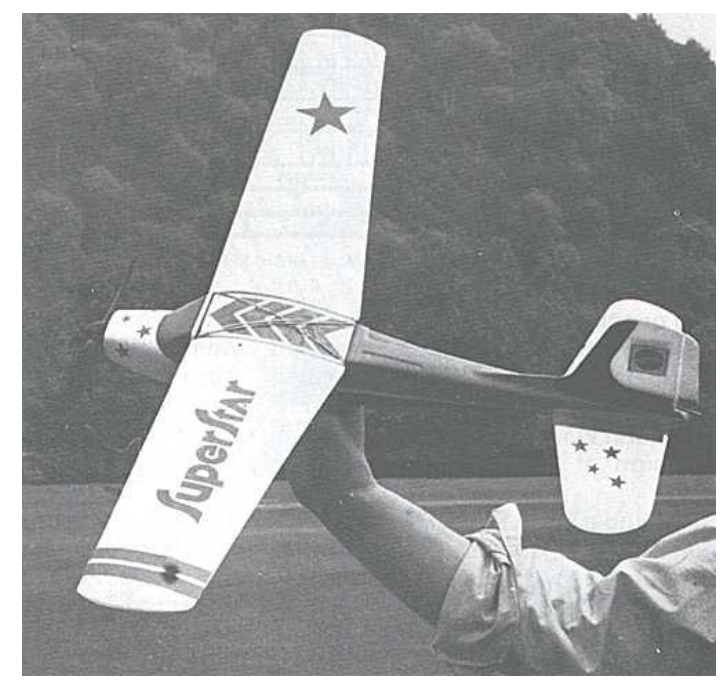

Figure 2 - Mattel SuperStar [10]

Advances in energy storage devices over the last 50 years have drastically affected our ability to apply electric propulsion to air vehicles. The main difficulty in flying an aircraft electrically is that the power sources have very low energy densities. Through the early 1990's, the best batteries available were nickel-cadmium or nickel metal-hydride. It wasn't until 1991 when lithium-ion batteries were released that the technology really saw a drastic increase in performance. Lithium-ion batteries were then modified to use a composite solid electrolyte. The resulting lithium-polymer batteries were introduced in 1996. Several characteristics of these batteries are included in Table 1. Of particular interest to aircraft applications is the energy density of the battery. Compared to the early nickel-cadmium based cells' $30-80 \mathrm{~W}-\mathrm{hr} / \mathrm{kg}$, the lithium technologies offers drastic increases, potentially as high as $200 \mathrm{~W}-\mathrm{hr} / \mathrm{kg}$ [11]. 
Table 1 - Characteristics of Common Batteries [11]

\begin{tabular}{|l|l|l|l|l|}
\hline Characteristic & NiCd & NiMH & Li-Ion & Li-Po \\
\hline Energy Density & $40-60 \mathrm{~W}-\mathrm{hr} / \mathrm{kg}$ & $30-80 \mathrm{~W}-\mathrm{hr} / \mathrm{kg}$ & $160 \mathrm{~W}-\mathrm{hr} / \mathrm{kg}$ & $130-200 \mathrm{~W}-\mathrm{hr} / \mathrm{kg}$ \\
\hline Energy / Vol. & $50-150 \mathrm{~W}-\mathrm{hr} / \mathrm{L}$ & $140-300 \mathrm{~W}-\mathrm{hr} / \mathrm{L}$ & $270 \mathrm{~W}-\mathrm{hr} / \mathrm{L}$ & $300 \mathrm{~W}-\mathrm{hr} / \mathrm{L}$ \\
\hline Power Density & $150 \mathrm{~W} / \mathrm{kg}$ & $250-1000 \mathrm{~W} / \mathrm{kg}$ & $1800 \mathrm{~W} / \mathrm{kg}$ & $2800 \mathrm{~W} / \mathrm{kg}$ \\
\hline Cycle Eff. & $70 \%-90 \%$ & $66 \%$ & $99.90 \%$ & $99.80 \%$ \\
\hline Lifetime & - & - & $24-36 \mathrm{mo}$. & $24-36 \mathrm{mo}$. \\
\hline Life Cycles & $2000 \mathrm{cycles}$ & $500-1000$ cycles & $1200 \mathrm{cycles}$ & $>1000 \mathrm{cycles}$ \\
\hline Nominal Voltage & $1.2 \mathrm{~V}$ & $1.2 \mathrm{~V}$ & $3.6 \mathrm{~V}$ & $3.7 \mathrm{~V}$ \\
\hline
\end{tabular}

More recently, the advent of lithium-sulfur rechargeable batteries has pushed the envelope of energy density out to $350+\mathrm{W}-\mathrm{hr} / \mathrm{kg}$ [12], some sources citing as high as 400 . Unfortunately, the lithium-sulfur batteries currently exhibit a cycle life of around 100 cycles. For HALE UAV applications with mission goals in the vicinity of days to months of persistent flight, these batteries are promising. If, however, desired endurance is on the scale of years, as with the DARPA Vulture program, lithium-sulfur batteries are not quite so attractive. On the positive side, advances in energy density are historically accompanied by a decrease in cycle life initially, until design refinement brings it back to acceptable levels [13] so we may expect improvements in the coming years.

When implemented into an aircraft design environment, the energy density of the batteries, or other energy storage systems, becomes one of the primary indicators of technology level, and represents a major determinant of aircraft weight. Another method of energy storage applicable to electric aircraft is the use of fuel cells. In October 2007, 
NASA presented a feasibility study focused on "Solar Airplanes and Regenerative Fuel Cells" in which they evaluate the energy storage requirements for year-long continuous flight. Of course, latitude has an effect on available solar energy, and the required energy density ranged from $250 \mathrm{~W}-\mathrm{hr} / \mathrm{kg}$ at the equator to $500 \mathrm{~W}-\mathrm{hr} / \mathrm{kg}$ at $45 \mathrm{deg}$. North [14].

\section{Aeronautical Solar Technology}

Supplying energy to onboard storage systems in all electric HALE aircraft must be performed by some type of environmental energy collection system. Many, if not all current HALE concepts employ solar power, and its use dates back to1974 when Sunrise $I$, lifted off of a dry lake bed in California for a 20 minute solar powered flight. This $27 \mathrm{lb}$ photovoltaic aircraft flew to $328 \mathrm{ft}$ above ground with 450 watts of supplied solar power. After sustaining damage during flight, an improved Sunrise II was constructed using higher efficiency solar cells and lighter structure, increasing the power to weight ratio from $16.6 \mathrm{~W} / \mathrm{lb}$ to $26.6 \mathrm{~W} / \mathrm{lb}$ [9]. Figure 3 shows Sunrise II on the lakebed.

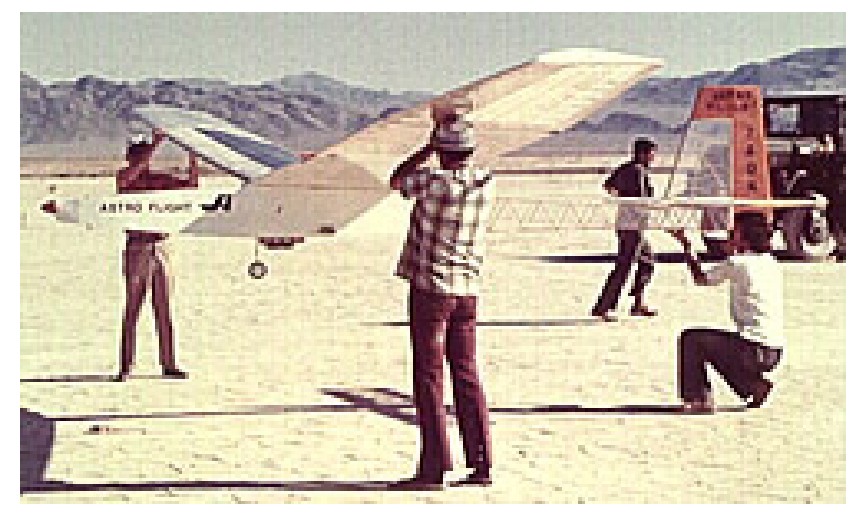

Figure 3 - Sunrise II Flight Preparation [9]

Photovoltaic technology as we know it today appeared first in 1954 with the development of the silicon photovoltaic cell at Bell Telephone Labs [15]. The first to be able to convert enough energy from the sun to run conventional electronics, this cell 
initially exhibited a $4 \%$ efficiency. Another major contributor to photovoltaic technology development was Hoffman Electronics, rolling out a 9\% efficient cell in 1958, followed by $10 \%$ in 1959 and $14 \%$ in 1960 . Development continued as more industries realized the potential of solar energy, and in 1964 NASA launched the Nimbus satellite powered by a 470 watt solar array [15]. As stated previously, the first application of photovoltaic technology to aircraft resulted in the successful flights of Sunrise I and II, shortly followed in Europe by Fred Militky and Solaris. Development of small to medium scale solar UAVs continues to the present, and in 2005, Alan Cocconi flew Solong, a $23 \mathrm{lb}$ solar regenerative power UAV that demonstrated 48 hour continuous flight [16]. Figure 4 shows Solong's $15.6 \mathrm{ft}$ wingspan aircraft as it would land, with the propeller blades folded aft.

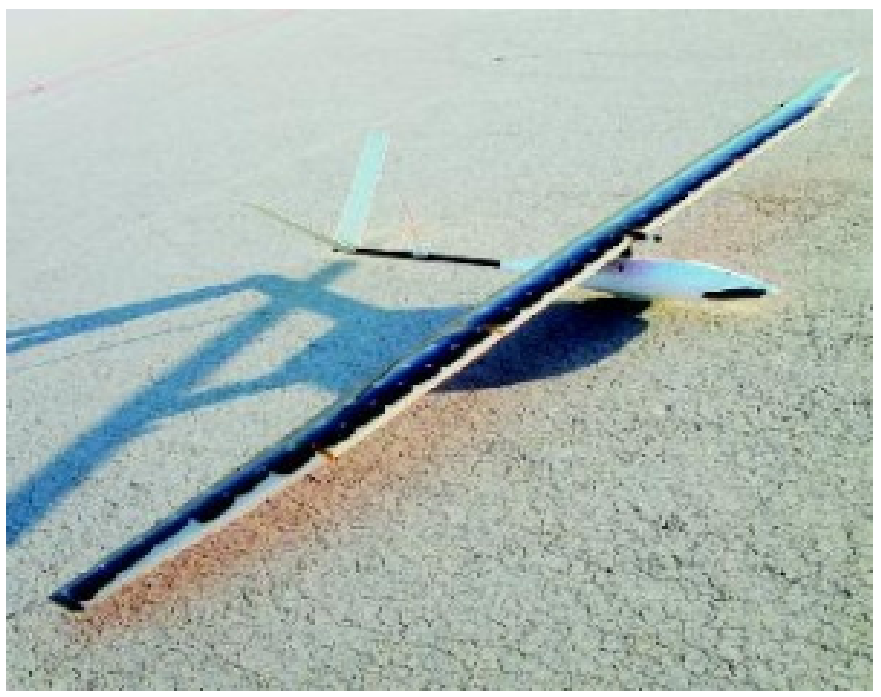

Figure 4 - Solong Multi Day Solar Aircraft (http://machinedesign.com) 


\section{Large Solar Aircraft}

Up to this point, most electric aircraft discussed have been small UAVs, with payloads ranging from zero to a couple pounds. The question remains, is this a promising area to explore larger payloads and aircraft? DARPA has expressed the desire for a long endurance aircraft that can support a payload of $1000 \mathrm{lb}$ and $5 \mathrm{~kW}$ [5]. Also, manned solar flight represents an environmentally friendly option for travel and recreation. Exploration into larger solar aircraft platforms began with Dr. Paul MacCready and AeroVironment Inc. Initially, Dr. MacCready explored man-powered flight with the Gossamer Condor and Gossamer Albatross [17]. These aircraft relied on advanced lightweight structures and an extremely low wing loading to achieve flight given low power available from the power plant (pilot). Expanding the aircraft design and construction techniques to the solar arena, MacCready developed the Gossamer Penguin (Figure 5), a smaller version of the Albatross with added solar panels.

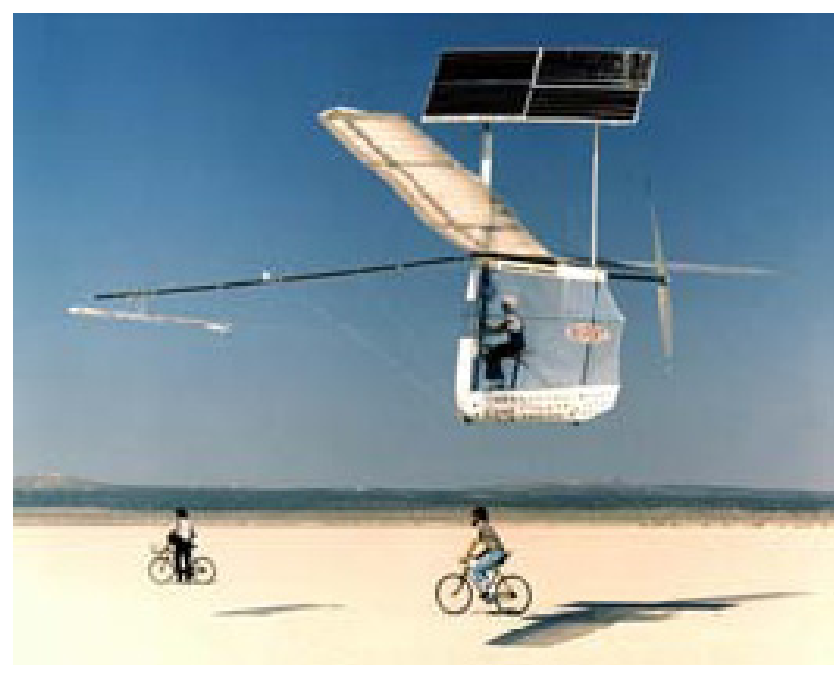

Figure 5 - The Gossamer Penguin in Flight [9]

R.J. Boucher, designer of the Sunrise UAVs worked with MacCready, supplying parts from the nonoperational Sunrise aircraft [9]. On May 18, 1980 the Gossamer Penguin 
became the first manned solar aircraft to demonstrate flight. DuPont, the sponsor for the Penguin, continued to support this concept by funding Solar Challenger, an aircraft designed by MacCready to cross the English Channel. On July 7, 1981, Solar Challenger completed the mission, flying for over 5 hours with no energy storage devices [9].

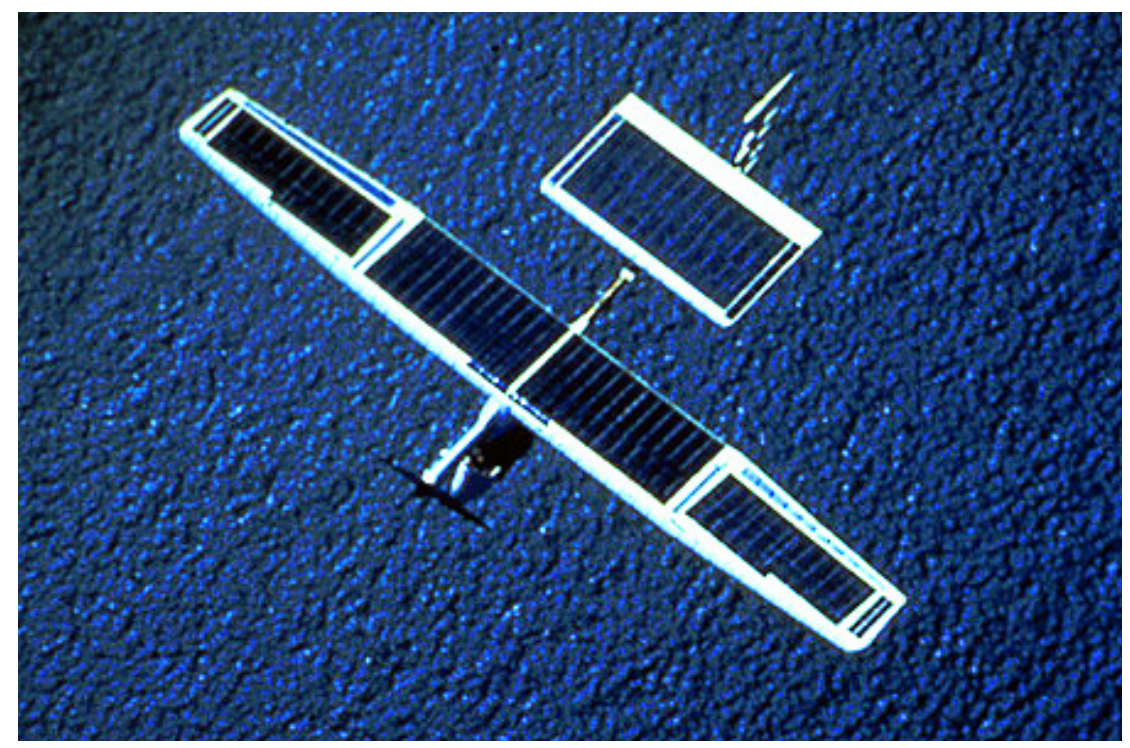

Figure 6 - Solar Challenger Flying Over the English Channel

Investigation into solar HALE aircraft as platforms for surveillance, communications, or other related missions began after Solar Challenger demonstrated the feasibility of solar power for aircraft. This along with the potential for relocatable and maintainable pseudo-satellites in the atmosphere spurred the development of $H A L S O L$ (High ALititude SOLar vehicle). The goals for the program were to fly above $65,000 \mathrm{ft}$ during day/night operations. The $440 \mathrm{lb}$ aircraft completed several validation flights under battery power but was unable to close the loop for solar regenerative energy and day/night operation and the program ended in 1983 [18]. 
Lessons learned from HALSOL were compiled and in the early 1990's, the Ballistic Missile Defense Organization funded an effort to update the airframe with modern technologies. Wing structures were modified and new solar cells, motors and propellers were added. The revamped airframe weighed in at $560 \mathrm{lbs}$ and was dubbed Pathfinder. In 1995 it set an altitude record for solar-powered aircraft at 50,500 ft. and two years later, the aircraft set a world altitude record for propeller driven aircraft at $71,530 \mathrm{ft}[18],[19]$.

Pathfinder was the first aircraft being evaluated under NASA's Environmental Research Aircraft and Sensor Technology (ERAST) program. ERAST was designed to develop and evaluate new technologies in sensors, light structures, aerodynamics, and propulsion to support extreme altitude and extreme endurance aircraft configurations [20]. With the success of Pathfinder, NASA and AeroVironment built a family of aircraft over the subsequent decade (Figure 7). Each new aircraft exhibited increased wing aspect ratio and more advanced power and propulsion technologies.

Pathfinder (1981-1997)

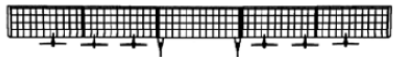

Pathfinder Plus (1997-1998)

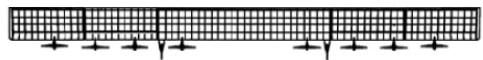

Centurion (1996-1998)

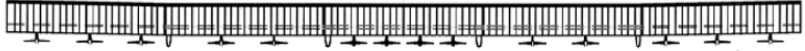

Helios Prototype (HP01), High-Altitude Configuration (1998-2002)

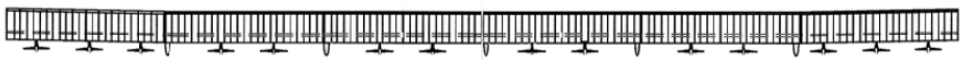

Helios Prototype (HP03), Long-Endurance Configuration (2003)

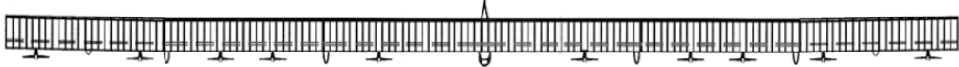

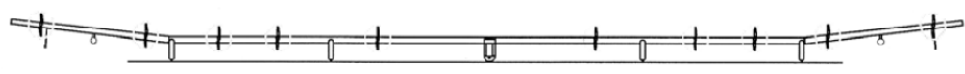

Figure 7 - Solar Aircraft in the ERAST Program [20] 
Culminating the ERAST program was Helios, the fourth and fifth generations of ERAST aircraft. Two aircraft were constructed; the first was optimized for high altitude (Figure 8) and the second for long endurance. NASA's goals for Helios were twofold. First, the aircraft was to demonstrate sustained flight above $100,000 \mathrm{ft}$, and second, it was to sustain flight for 24 hours with at least 14 of those above 50,000 ft altitude. In 2001, the high altitude configuration of Helios reached a world record altitude of 96,863 ft. and flew for 40 minutes above $96,000 \mathrm{ft}$ [21]. Unfortunately, the redistribution of weight for the long endurance configuration included a large point mass at the midpoint of the wing to house fuel cell equipment. What resulted was an aircraft that didn't exhibit the same qualities of a span loaded aircraft as its predecessors, and several control algorithms encountered errors during a persistent high wing dihedral. The aircraft became unstable in pitch and was destroyed in flight over the ocean [20].

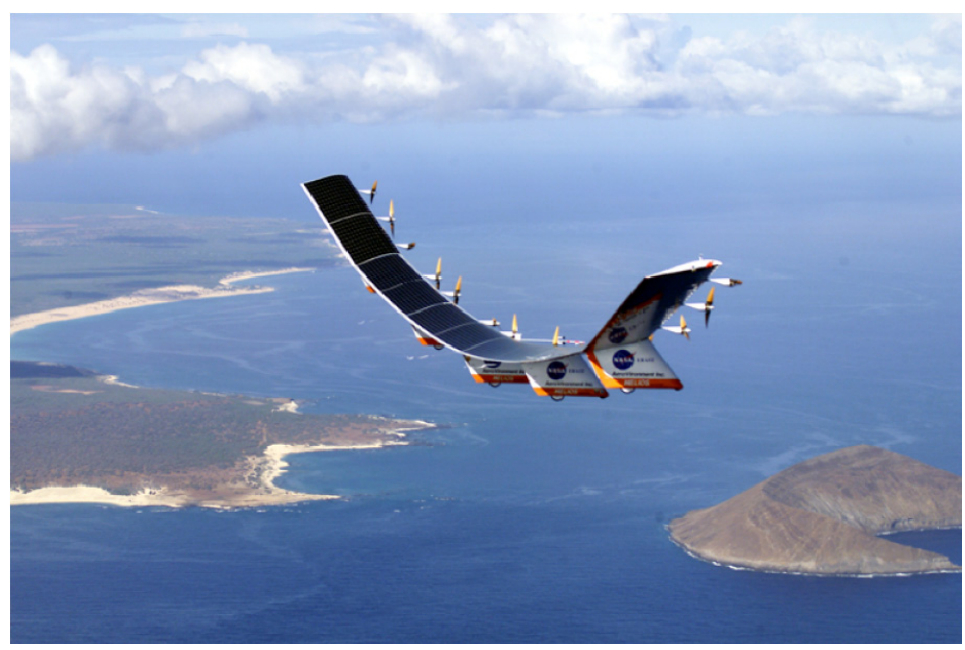

Figure 8 - Helios High Altitude Configuration [20] 


\section{Extreme Aspect Ratio Concept}

Developing a revolutionary concept for increasing aspect ratio without paying the penalties commonly associated with doing so is primarily inspired by a somewhat obscure Air Force research effort in the early 1950s that was itself inspired by German scientist Dr. Richard Vogt who emigrated to the U.S. after WWII. The initial concept was that the range of a bomber may be increased by adding "free-floating" wing segments that are pinned to the bomber wingtips [22]. Also, the U.S. military wanted to examine the feasibility of utilizing the long range capabilities of bombers like the B-36 Peacemaker or the B-29 Superfortress to tow, carry, or otherwise transport smaller and more maneuverable fighter aircraft like the F-84 to foreign combat zones. The first concepts involved the smaller aircraft docking in the bomb bay of the bomber for parasitic flight to and from the target. In this scenario, the extra aircraft adds parasitic drag to the bomber, and takes up bomb bay space, while not contributing anything aerodynamically positive in return [23]. A follow-on effort designated MX-1016 "Tip Tow" moved the parasite fighter from under the host aircraft to the wingtip [22]. Figure 9 shows a Boeing B-29 in flight with two EF-84 aircraft coupled at the wingtips [24].

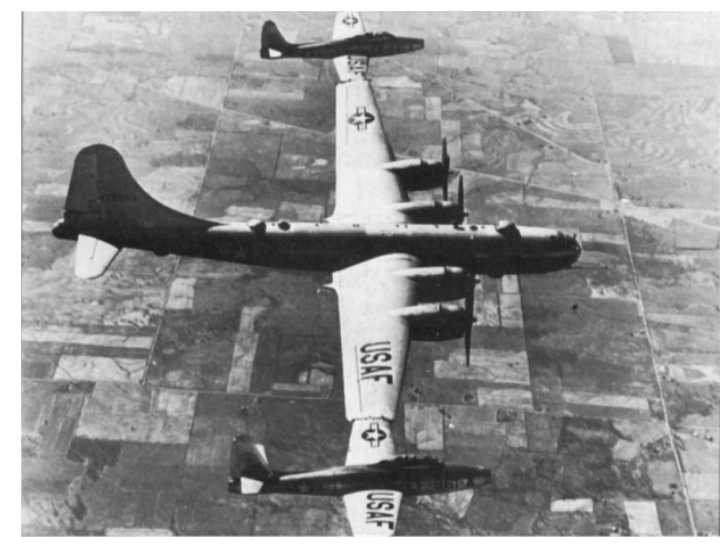

Figure 9 - Early Air Force Wingtip Coupled Flight [24] 
With the new configuration, the parasitic aircraft now contribute additional wingspan to the bomber, reducing the induced drag [25]. The wingtip coupling mechanisms underwent some revision under a new project called "Tom-Tom" involving clamps or jaws on the wingtips of a B-36 [24].

Applying the idea of wingtip coupling to a flying wing HALE aircraft represents a revolutionary step in the field. Conceptually, each segment of the flying wing would lift its own weight and comprise a generally self-sufficient aircraft system. Stringing some number of individually moderate aspect ratio wing segments together to form an extreme aspect ratio platform allows each segment to benefit from lower structural loads while simultaneously reaping benefits of an extremely high aspect ratio planform. Figure 10 shows what one such vehicle might look like.

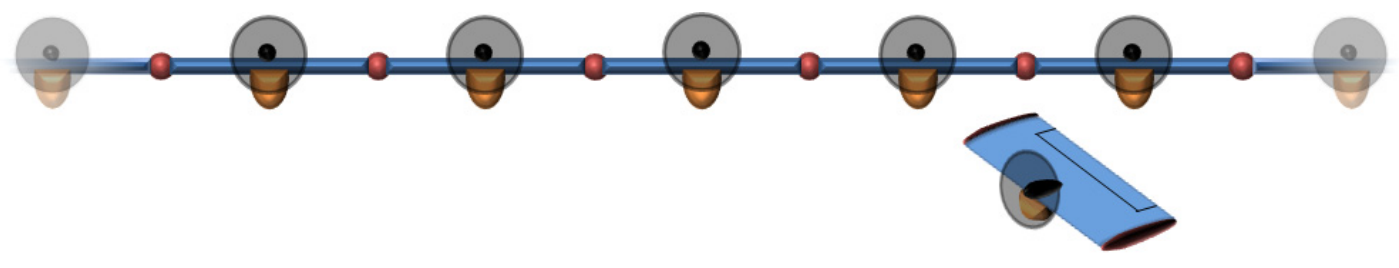

Figure 10 - Extreme Aspect Ratio Concept

In addition to lowering the structural weight fraction of the aircraft, a "pinned wing" concept may offer the ability to orient solar cells favorably towards the sun. Also, with a conventional high aspect ratio flying wing configuration, natural frequencies of the structure can be so low that they approach control response frequencies. If these come too close together, an aileron deflection, or step input may induce structural resonance rather than the desired change in flight condition. Considering that the individual wing segments of the extreme aspect ratio concept have low-to-moderate aspect ratios, they will be more rigid and exhibit higher natural bending mode frequencies. 


\section{PROBLEM STATEMENT}

The central goal of this research is the development of a multidisciplinary tool for analysis, design, and optimization of High Altitude Long Endurance (HALE) UAVs. Current and projected future missions for this type of aircraft platform focus on its ability to provide sustained support for surveillance, communications, or other science missions, and act as an "atmospheric satellite". Accordingly, the baseline mission profile consists of a climb to some stratospheric cruise/loiter altitude, where the aircraft begins mission operations and enters an extended cruise or loiter flight mode, shown in Figure 11.

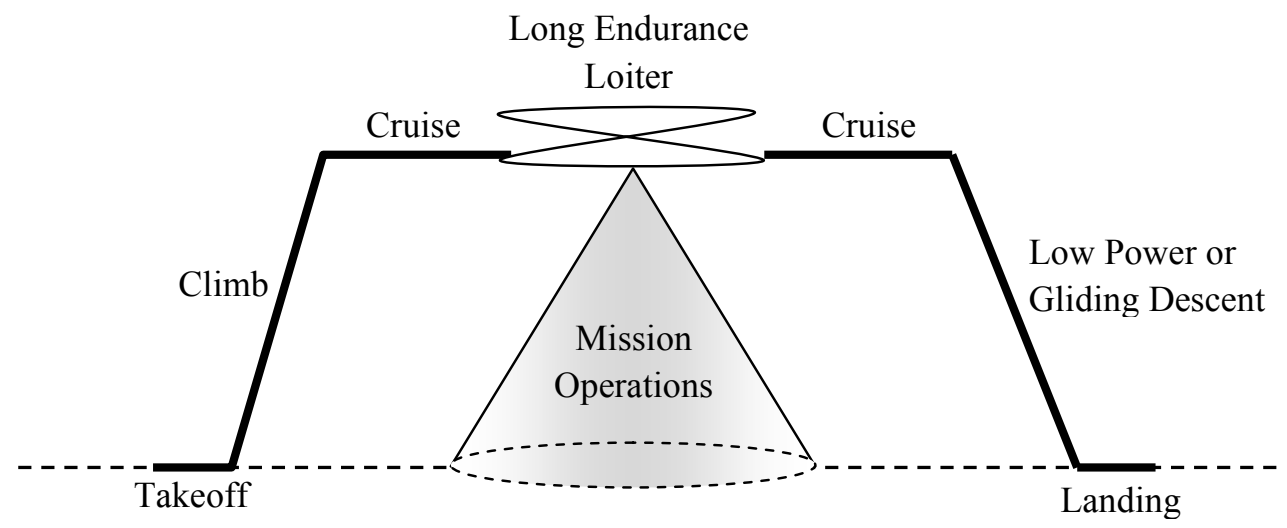

Figure 11 - HALE UAV Mission Profile

With an integrated design and analysis environment in place, certain parameters may be adjusted to approximate current or past proven aircraft configurations in an effort to calibrate the tool. Next, optimization techniques are applied to a baseline platform, here represented by flying wing aircraft similar to Helios or the Vulture concept. Investigation of performance trends and the effect of new technologies, as well as system sensitivities from parametric studies may be performed at this stage. Considerations are made throughout the development of the tool to allow a wide range of missions, payload systems, and potential solutions. 
Following the analysis and optimization of a proven platform aircraft, the design tool is adjusted to study the effect of implementing a segmented wing concept. Again, the individual flight science modules, or discipline analysis codes are developed to be flexible and applicable to both single and multiple segment wings. As before, parametric studies and optimization of the segmented platform reveal design drivers and best configurations, which may be compared with the results from the proven aircraft study, as well as actual flown aircraft results.

One of the attractive aspects of a segmented wing platform is the ability to build it up from identical and self sufficient segments, allowing more flexibility for payloads and missions. As such, the optimization objective is to develop a platform of identical segments that minimizes aircraft weight while meeting mission, performance, and technology constraints. In reality, the goal is minimization of total cost, which may be composed of both acquisition and operating cost. For the included studies, aircraft cost is assumed to be well represented by aircraft weight, as suggested in [26]. Also, in a report prepared for the Suborbital Science Office Earth Science Enterprise of NASA, it is proposed that a breakthrough in reducing acquisition cost of UAV science missions may be possible with a new generation of small HALE aircraft with simplified operational requirements [27]. It is important, however, to consider that all-electric HALE platforms are pushing the frontier of energy technology, and parameters like the specific energy density of batteries, or efficiencies of power system components like solar cells, power conditioning units, speed controllers and motors may greatly affect costs. It is possible to consider alternative objectives for the optimization that may account for the cost dependence on technology, such as minimizing required solar efficiency for a mission. 


\section{METHODOLOGY}

Development of the multidisciplinary analysis, design, and ultimately optimization tool begins with a buildup of individual disciplines, each capable of analyzing both a baseline flying wing/cantilever configuration and a segmented wing platform. What follows is a description of the approach to optimization, a look into the integrated multidisciplinary environment, and specifics about the individual disciplinary analysis methods.

\section{Optimization Architecture}

With aircraft weight as the central objective for minimization, parameters describing the aerodynamic, structural, propulsive, and energetic qualities of the aircraft are varied to determine preferred configurations that meet applicable constraints. As the main body of code was developed in MATLAB, the built-in optimizer "fmincon" is used to minimize a constrained multivariate function. The general form of the optimization problem is given in (1).

$$
\min _{\vec{x}} f(x) \text { such that }\left\{\begin{array}{c}
c(\vec{x}) \leq 0 \\
\operatorname{ceq}(\vec{x})=0 \\
l b \leq \vec{x} \leq u b
\end{array}\right.
$$

Where $c$ represents a set of inequality constraints, $c e q$ represents the equality constraints, supplemented by $l b$ and $u b$, the lower and upper bounds enforced on the set of design variables $\vec{x}$.

When performing an optimization, fmincon defaults to attempt to use a trustregion-reflective algorithm, which requires a user supplied gradient for the objective 
function. Developing an analytical gradient for the entire design process for an aircraft from conceptual design through mission analysis and preliminary sizing is both difficult and beyond the scope of the work herein. In addition, several of the analysis modules utilize pre-compiled binaries or executables, further discouraging any attempt to find the gradient. Instead, an optimization algorithm must be used that numerically estimates gradient and Hessian functions. The Optimization Toolbox in MATLAB offers ActiveSet optimization for problems such as this. For Active-Set optimizations, MATLAB implements sequential quadratic programming (SQP) to choose search directions by mimicking Newton's method [28]. Sequential Quadratic Programming approximates the objective function, generally $\mathrm{W}_{\text {tot }}(\vec{x})$, as a quadratic function. The method then linearizes constraints locally and applies Quadratic Programming to approximate the solution. SQP looks to the Broyden-Fletcher-Goldfarb-Shanno (BFGS) method for updating the Hessian [29].

Fundamentally, the process employed for designing the UAV is an iterative process, meaning that from a systems perspective, there is feedback inherent in the data flow structure. Specifics about the feedback quantities are discussed in the next section, but how they are handled effects the optimization environment. Two methods were considered and tested for solving the resulting system of nonlinear equations. The first is a simple iterative scheme that converges all of the system feedback given initial guesses for each feedback quantity. Generally referred to as Fixed Point Iteration (FPI), this scheme provides an intuitive method for solving a system, but no guarantee that a solution exists. An attempt was made at developing a convergence criteria using Newton's Method, but an analytical representation of the whole system is not feasible. 
The alternative to FPI is a process called Optimizer Based Decomposition (OBD) where data links in the system of equations are broken and replaced by new design variables and constraints [30], [31]. Specifically, for the problem of interest here, only the feedback data links are decomposed, and we designate the process Partial OBD (POBD). Allowing the optimizer to simultaneously handle the regular design variables and the requirement for a converged system decreases the run time per iteration and increases the probability of closing the design, or achieving convergence. In addition, the constraints on convergence may be held strict or loosened depending on the desired fidelity of the solution. A simplified and purely theoretical system is presented in Figure 12 to illustrate the interaction between disciplines. In this system representation, active column elements are inputs to a module and rows are outputs. Reference [32] gives a thorough description of the processes for using a diagram like this, but completing a quick system trace reveals that active cells in the lower triangle represent feedback data paths. A POBD process operates on these cells to eliminate the need for iterative solving.

\begin{tabular}{|c|c|c|c|c|c|}
\hline Geom. & $\mathrm{x}$ & & $\mathrm{x}$ & & \\
\hline & Aero & $\mathrm{x}$ & & & $\mathrm{x}$ \\
\hline & $\mathrm{x}$ & $\begin{array}{c}\text { Mission } \\
\text { Analysis }\end{array}$ & $\mathrm{x}$ & $\mathrm{x}$ & $\mathrm{x}$ \\
\hline $\mathrm{x}$ & $\mathrm{x}$ & $\mathrm{x}$ & Weights & $\mathrm{x}$ & $\mathrm{x}$ \\
\hline & & & & Cost & \\
\hline & & & & & Perf. \\
\hline
\end{tabular}

Figure 12 - Theoretical Aircraft Design Structure Matrix

A more detailed Design Structure Matrix (DSM) is presented in the next section, and represents the actual multidisciplinary system implemented for this study. 


\section{Design Variables}

Parameters selected as design variables for this optimization are products of the approach to conceptual design, mission analysis, and preliminary aircraft design methods discussed in the next section. As a quick preview, several key characteristics of the aircraft are specified up front, and an iterative design process (hopefully) converges to a final configuration. A complete list of inputs to the design environment comprises the set of all potential design variables, only some of which are actually selected as design variables for the optimizer, while others represent technology factors, mission characteristics, or configuration identifiers. The design of experiments for a complex multivariate optimization problem such as this begins with selecting a basic set of design variables, leaving the rest as constants that define aspects of the configuration. Table 2 shows the basic set of design variables used.

Table 2 - Initial Optimizer Design Variables

\begin{tabular}{|l|l|}
\hline & Design Variable \\
\hline 1 & Total aspect ratio \\
\hline 2 & Wing loading $\left(\mathrm{lb} / \mathrm{ft}^{2}\right)$ \\
\hline 3 & Power to weight $(\mathrm{W} / \mathrm{lb})$ \\
\hline 4 & Wing thickness-to-chord ratio \\
\hline 5 & Percent of $\mathrm{S}_{\text {ref }}$ covered by solar panels \\
\hline
\end{tabular}

Along with the fundamental design variables of Table 2, there are several other parameters of the design which may be more effective as optimizer-controlled design 
variables than predetermined quantities. Table 3 shows these parameters, including the variables necessary for implementing POBD. The '+' symbol indicates that there are more than one actual variables associated with the characteristic, that variable can be thought of as a vector quantity. For example, the battery-pod spanwise locations may be left to be determined by the optimizer, but there may be 10 pods holding batteries or payload so that the actual design variable may have dimension [1x5] depending on symmetry assumptions.

Table 3 - Additional Design Variables

\begin{tabular}{|l|l|}
\hline & Design Variable \\
\hline $6^{*}$ & Total weight (lb) \\
\hline $7^{*}$ & Wing weight (lb) \\
\hline 8 & Cruise Altitude (ft) \\
\hline 8 & Payload weight (lb) \\
\hline 9 & Payload power requirement (W) \\
\hline $10+$ & Spar factor of safety, material properties \\
\hline $11+$ & Spar cross section locations (\% span) \\
\hline $12+$ & Battery or payload pod locations (\% spar) \\
\hline $13+$ & Technology factors \\
\hline & optimizer-based decomposition variable \\
\hline
\end{tabular}

Lastly, there are several characteristics of the aircraft that are discrete numbers. The optimization methods employed do not handle such data types, so when designing the experiments, each of the variables in Table 4 must be specified for a set of parametric 
studies or optimization runs. Results may then be compared between the discrete values to assess potential benefits against additional complexity.

Table 4 - Discrete Variables

\begin{tabular}{|c|}
\hline Number of wing segments \\
\hline Number of battery/payload pods \\
\hline Number of spar cross sections \\
\hline
\end{tabular}

\section{Optimizer Constraints}

Equation (1) states that our system may be subject to either inequality or equality constraints which may act on the design variables themselves or certain determined quantities within the system. Decisions about which metrics to use as constraints are a bit more vague with a HALE UAV platform than with aircraft designed for more conventional missions. Take, for example, the recent Broad Agency Announcement delivered by DARPA requesting proposals for a HALE UAV. The requirements supplied by DARPA are simple and few [5]:

- 5 years uninterrupted operation

- $1000 \mathrm{lb}, 5 \mathrm{~kW}$ payload

- $99 \%$ probability of station-keeping

- High probability of mission success

From a preliminary design point of view, the first two bullets are the only requirements. Where other categories of aircraft may have a set of point performance requirements 
explicitly laid out for them, in our case the designer must work diligently to flow down these two top-level requirements into subsystems requirements and ultimately design constraints.

Considering another recent HALE platform that broke new ground for its kind, a general goal for qualifying as 'high altitude' can be defined. The high altitude configuration of Helios (HP01) was designed with the intent of demonstrating flight at $100,000 \mathrm{ft}$.

From these reference programs, the central desired capabilities of future HALE UAVs is distilled into the first two constraints imposed on the optimization environment herein. First, we impose the requirement that the all-electric aircraft must achieve a sustainable energy balance for repeatable day/night operation. Persistent multi-day operation necessitates the inclusion of both energy generation and energy storage systems. This first constraint requires that for a given flight profile, the aircraft must be able to generate enough power during daytime operation to not only sustain flight and payload operations, but to do so with enough excess energy produced to power the aircraft through the night. In addition, the aircraft must be able to support the weight of a system capable of storing this excess energy, along with any associated power management systems. When considering batteries as the storage medium, current technologies result in as much as $30-50 \%$ of the total aircraft weight taken up by energy storage, meaning that the persistent operation requirement is a major design driver.

Supporting the payloads of interest for programs like Vulture or Helios requires stratospheric flight altitudes, and as previously stated, a good benchmark for future platforms is flight at 100,000 ft altitude. This becomes the second constraint imposed on 
the design environment, stating that the absolute ceiling of the aircraft must be at least $100,000 \mathrm{ft}$.

In order to keep the solutions controlled to a reasonable domain, a third constraint is imposed that defines a maximum wingspan. Initial studies showed that without this constraint, optimal configurations sometimes exhibited wingspans of nearly $500 \mathrm{ft}$, almost twice that of the Airbus A380. A constraint value of 300 feet is used for the majority of the study herein, and was chosen to be similar to the Helios aircraft with some room to grow.

The fourth and fifth constraints are products of the POBD of the design system. As implemented, two feedback variables have been offloaded onto the optimizer: total weight and the structural weight of the wing. For each function call, the optimizer provides initial guesses for these weights, allowing the design environment to calculate dimensional values for things like required energy or power, wetted area, solar panel area, etc... In addition, the structures module must account for the weight of the wing when sizing the spar. This catch-22 of needing a guess of structural wing weight in order to calculate the structural wing weight characterizes the feedback loop that was decomposed. Accordingly, convergence of the design is enforced by imposing equality constraints that require the calculated wing structural weight and total aircraft weight be within a certain tolerance of the guessed values. Table 5 summarizes the fundamental constraints imposed on the design and optimization environment. These constraints remain the same whether implementing single segment baseline configurations or multisegment XAR concepts. 
Table 5 - Fundamental Design Constraints

\begin{tabular}{|l|l|l|}
\hline & Constraint & Type \\
\hline 1 & Multi-day energy balance & Inequality \\
\hline 2 & Absolute ceiling & Inequality \\
\hline 3 & Wingspan & Inequality \\
\hline 4 & Total weight compatibility & Equality \\
\hline 5 & Wing structural weight compatibility & Equality \\
\hline
\end{tabular}

The HALE UAV optimization problem is expressed in standard form below to provide a general summary of the variables, constraints, and objective. When implemented, design variables, objective function values, and constraint values are all individually linearly scaled to have a magnitude on the order of $10^{0}$. This process is important because it helps to ensure well-conditioned Lagrange multipliers used in evaluation of constraints under methods like Kuhn-Tucker (KT) conditions [29].

minimize $\quad W_{\text {tot }}=f(\vec{x})$

$$
\text { where } \vec{x}=\left\{\begin{array}{c}
\text { Aspect ratio } \\
\text { Wing loading } \\
\text { Power to weight } \\
\text { Wing t/c } \\
\% \text { solar coverage } \\
W_{\text {tot }} \\
W_{\text {wing }} \\
\text { Payload } \\
\text { Technology } \\
\text { etc ... }
\end{array}\right.
$$




$$
\text { subject to } \begin{aligned}
\frac{E_{\text {batt_req }}+E_{\text {payload }}+E_{\text {systems }}-E_{\text {solar }}}{\operatorname{mag}(E)}<0 \\
1-\frac{h_{\text {absolute_ceiling }}}{100,000}<0 \\
\frac{b}{300}-1<0 \\
\frac{\left|W_{\text {tot_guess }}-W_{\text {tot_calc }}\right|}{\text { mag }\left(W_{\text {tot }}\right)}=0 \\
\frac{\left|W_{\text {wing_guess }}-W_{\text {wing_calc }}\right|}{\text { mag }\left(W_{\text {wing }}\right)}=0
\end{aligned}
$$

Lastly, the tool includes many parameters that may be rearranged to alter the optimization problem. These additional characteristics, when implementing the optimization described above, are either set as inputs to the system, or determined as outputs. However, with minor adjustments, the aircraft may be optimized for a different objective, and/or subject to alternative constraints. For example, additional constraints may be set for the number of motors, or the solar cell efficiency or energy storage density may be introduced as design variables and objectives for minimization.

The optimization architecture should be kept in mind for the remainder of the Methodology section. What follows is a description of each major analysis module in the MDO tool, and there are several instances where decisions are made or validation cases are run specifically because of the implementation as an optimization tool. 


\section{Multidisciplinary Integration}

Development of a multidisciplinary analysis, design, and optimization tool begins with the identification of which disciplines are involved, and what inputs and outputs are associated. Much of the aircraft design process involves coupled systems, feedback, and indirect dependencies that pose significant challenges to analytical modeling or sequential design. There are many approaches to initial concept design, sizing, and weight estimation for an aircraft, but many of the traditional methods have significant shortcomings when applied to the systems of interest here. The majority of classical preliminary design methodologies have three central tasks: point performance analysis (constraint diagram), mission analysis, and weight estimation (Figure 13).

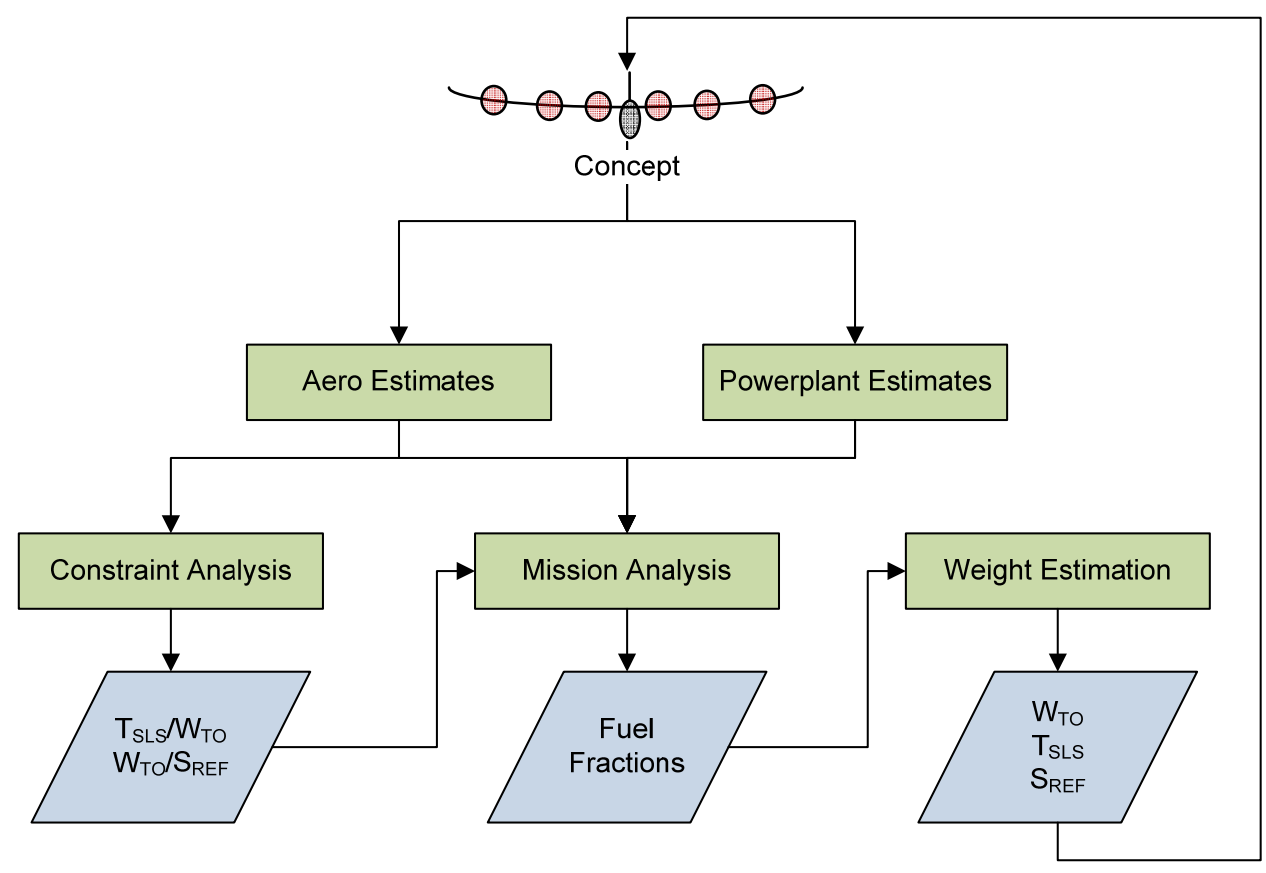

Figure 13 - Classical Aircraft Sizing Process 
When considering a revolutionary concept such as an all-electric HALE UAV under this sizing architecture, several problems arise. First, new propulsion and energy systems which depart from the internal combustion arena will surely beget unconventional configurations as seen with the development of the AeroVironment family of vehicles under the NASA Environmental Research Aircraft and Sensor Technology (ERAST) effort that led up to the Helios Prototype [21]. Sizing an aircraft according to Figure 13 requires some knowledge of weight trends, generally in the form of historical regressions or expert opinion concerning empty or structural weight fractions. Care must be taken in selecting these parameters, but with resources describing structural optimization of HALE aircraft, conventional sizing methods may still apply [1], [33], [34]. Alternatively, structural weight estimation may be achieved using more complex physics-based tools that find worse-case loading situations and size the major structural elements of the aircraft accordingly. This "bottom-up" method for estimating empty weight fractions is employed in the final MDO tool and is described later in this section.

The second, and more pronounced problem sizing an alternative fuel aircraft with the model of Figure 13 is the mission analysis. Currently, the traditional sizing algorithm requires a portion of the aircraft to "burn up" during the mission in the form of fuel weight; if the aircraft has no combustion cycle and consequently completes the mission with no weight change, the process of Figure 13 breaks down. This inflexibility to alternative methods of converting energy to power is the motivator for developing a new sizing process with one fundamental difference. Our new method of initial design will focus more directly on the energy of the aircraft without inherently selecting the form that 
energy occupies. For example, the mission analysis of our new design methodology does not calculate the fuel fraction required for climb. Instead we develop the total energy requirement for the climb (actually the total energy normalized by aircraft weight). At the completion of our mission analysis we will have developed the total mission specificenergy requirement represented in units of energy per pound of aircraft. This approach allows us to then apply any set of energy sources to the airframe including but not limited to batteries, fuel cells, photovoltaic generation, and conventional internal-combustion based power plants. A similar approach was taken in developing an Architecture Independent Aircraft Sizing Method (AIASM) by Dr. Taewoo Nam [35], and is supported by power system analysis given in [36].

With a sizing method applicable to electric-powered aircraft and a design perspective centered on energy, the foundation of a valid conceptual design environment has been laid. Building the MDAO capability around our new approach to sizing follows as it would for any optimization environment. A functional decomposition, or multilevel breakdown, of the aircraft system leads to the central disciplines that will be involved in the design process [7]. Figure 14 shows the specific areas of analysis that comprise the MDAO environment in the form of an $\mathrm{N}$-squared diagram. As pictured, the analysis modules have been arranged to minimize feedback, though it is still present. Feedback in the system is represented by links in the lower triangle of the matrix. As previously mentioned, these areas of feedback are disconnected and the requirement for design convergence is enforced by the controlling optimizer. What follows is a discussion of the modules in Figure 14 covering the inputs, outputs, and methods for each. Unless otherwise stated in the description, the modules were implemented in MATLAB. 


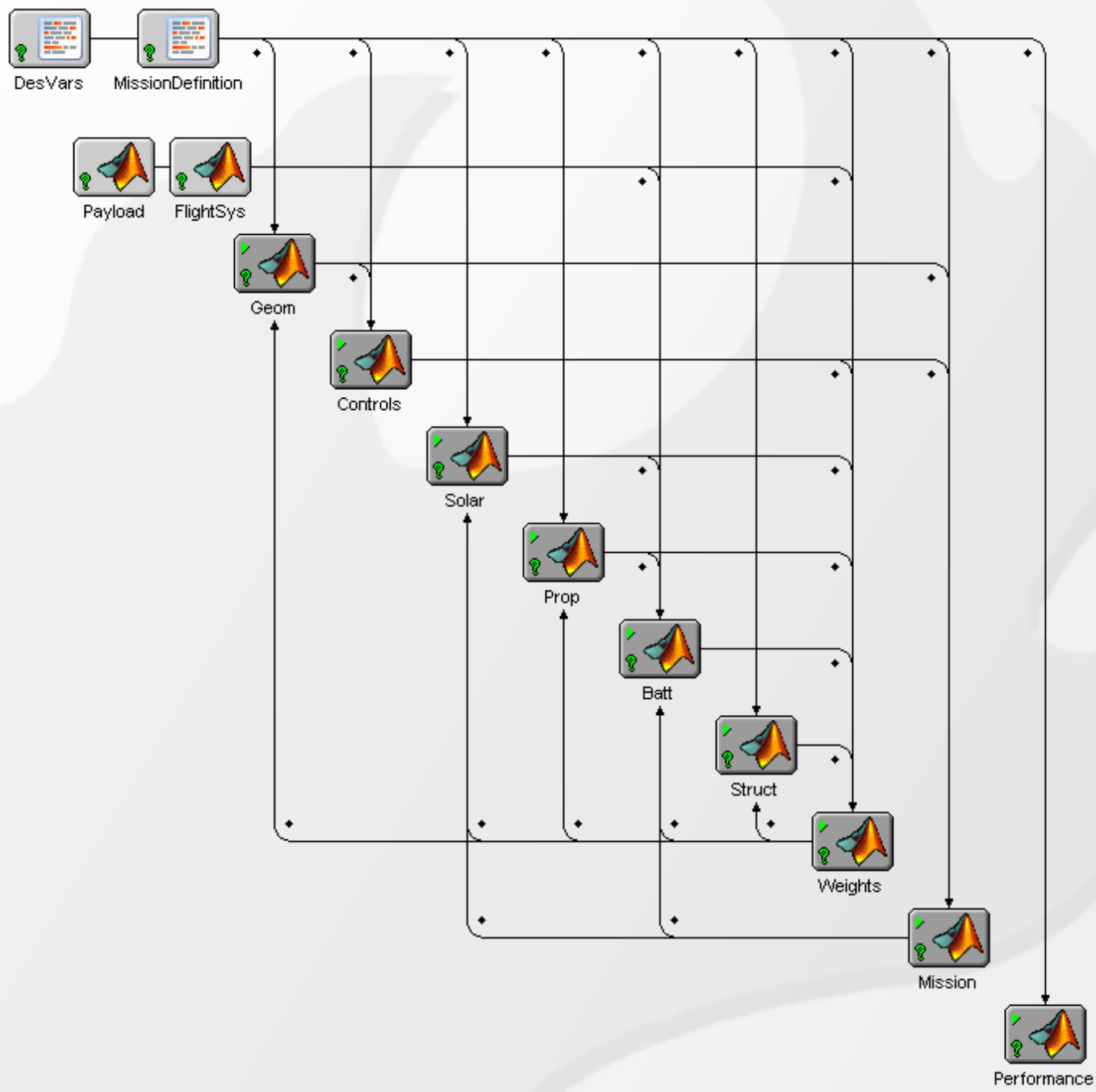

Figure 14 - Multidisciplinary Design Analysis and Optimization Architecture

\section{Mission Analysis}

Given a set of mission requirements or mission profile, the first step in sizing an aircraft is examining the energy or power requirements. With the all-electric HALE aircraft, the goal for this module is not only to find these values, but also to act in a flight planning capacity. This module optimizes flight $C_{L}$ at each mission segment for either minimum power or minimum energy required. 
Performing such analysis for a long endurance electric aircraft is slightly different than for an aircraft with a specific desired range or endurance, or one with a combustionbased power plant. With the Vulture specifications in mind, one of the fundamental goals for our design is to fly as long as possible, and considering that not every configuration will be able to achieve sustained day/night operation, we really do not know how long the cruise or loiter mission segments will last. The desired output of this module is the energy requirement for the platform, but we may not know the mission time, so the energy must be normalized by time, resulting in required power as an output for certain mission segments rather than required energy. Also, we want to be able to perform this analysis for a non-dimensional aircraft, so all of the internal processes are normalized by weight, resulting in outputs of specific energy (Watt-hr/lb) or specific power (Watt/lb) required.

Without the need for fuel fractions, the actual analysis of the mission may be completed in a straightforward manner from a physics-based approach rather than using the empirical formulas found in many classical design texts. This process was completed at two levels of fidelity. Initially, the mission was modeled in full by integrating the equations of motion for an aircraft in the x-z plane over time. For different segments of the mission, the flight planning process selects desired flight conditions and the resulting power is integrated to find energy and time. MATLAB's built-in ODE solver, ODE45, was used here. While this method provides high fidelity estimates of the mission, the numerical integration process is time intensive, and when running the integrated design tool, this module took substantially more time than others.

In an effort to provide faster estimates, the method was revised to simplify the analysis. Each mission segment like takeoff or cruise is broken up into some number of 
sections, for example, we may model climb with three discrete flight conditions rather than one at each time step. Figure 15 shows the implementation of this approximation on a general flight envelope for a HALE vehicle.

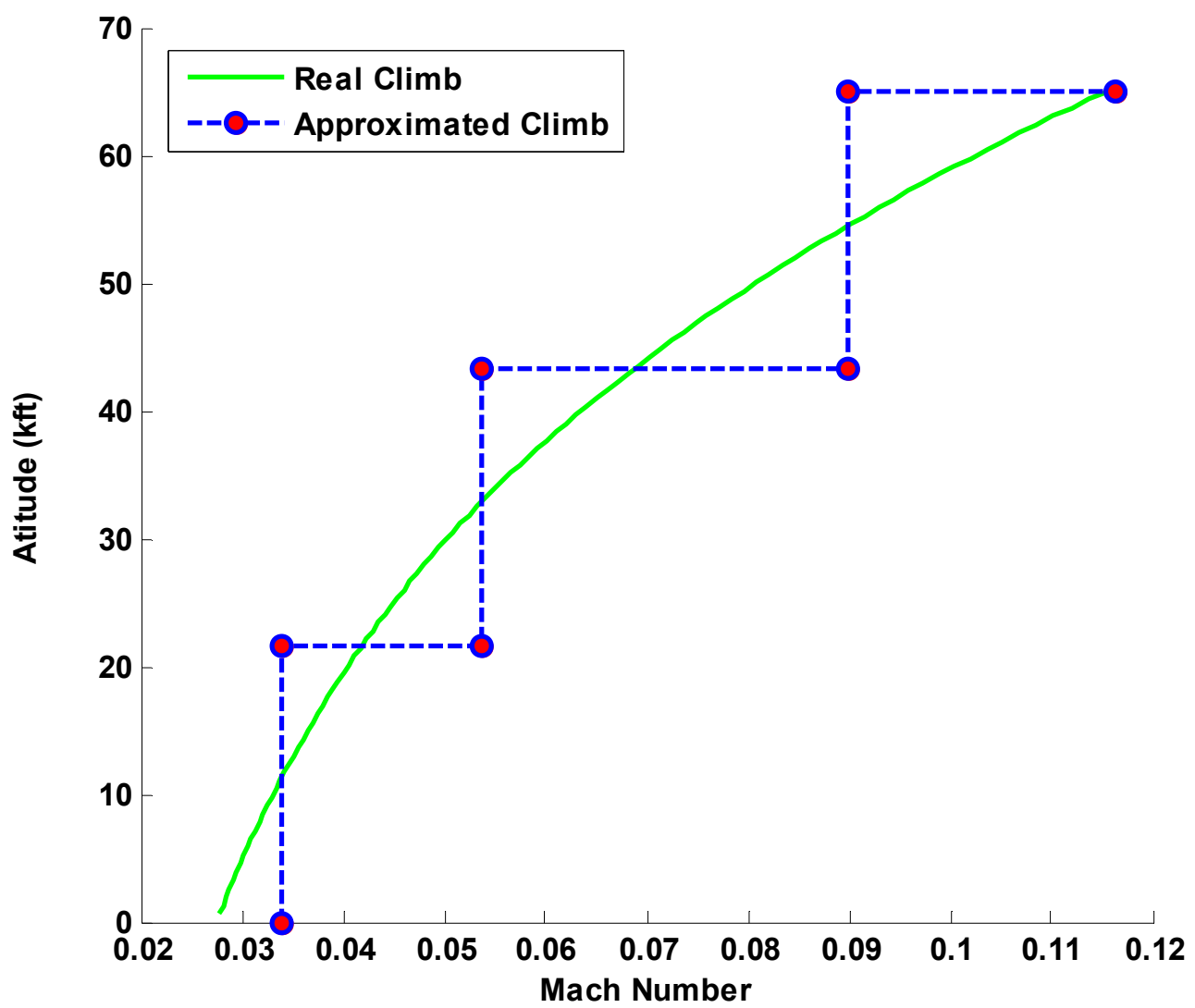

Figure 15 - Approximation of Climb

At each of the three segments, we calculate how long it would take to climb to the next segment, and how much energy would be required to fly at that speed for that amount of time while gaining altitude or increasing energy height of the system. Figure 16 shows how the accuracy of this approximation method is affected by the number of discrete steps the climb is broken into. The number of climb segments is a parameter that may be changed by the user, and is set at 20 for studies presented in this report. 


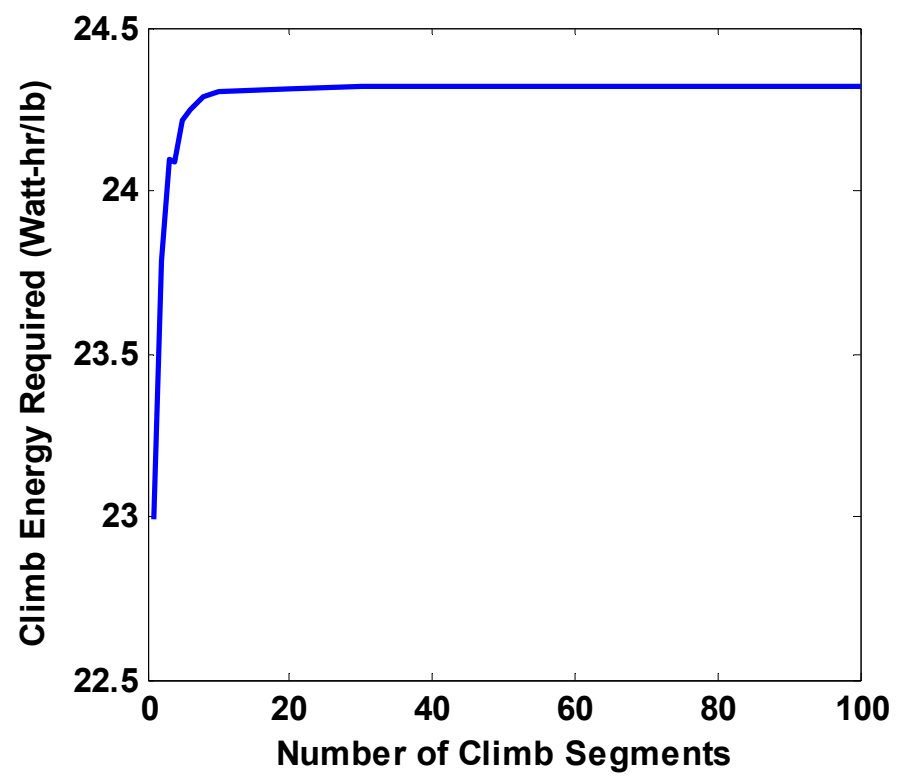

Figure 16 - Comparison of Mission Analysis Methods

The communication requirements for the mission analysis portion of the multidisciplinary environment are listed in Table 6. For mission legs with known durations, the power and time outputs can be combined into the required specific energy.

Table 6 - Mission Analysis I/O

\begin{tabular}{|c|c|}
\hline \multicolumn{2}{|c|}{ Mission Analysis Module } \\
\hline Inputs & Outputs \\
\hline Wing loading & Climb specific power req. \\
\hline Power to weight ratio & Cruise specific power req. \\
\hline $\mathrm{C}_{\text {Lmax }}$ & Loiter specific power req. \\
\hline Propeller efficiency & Time for climb \\
\hline Mission profile & Time for cruise \\
\hline \# segments for climb approx. & Best flight $\mathrm{C}_{\mathrm{L}}$ 's \\
\hline
\end{tabular}




\section{Aerodynamics}

The aerodynamics module of the design tool is tasked with developing drag estimations for our various flight conditions, but doing so for geometries that may vary drastically in scale. Our resulting drag polar relies on two methods, one evaluates lift distribution and induced drag, and the other estimates elements of aircraft drag polars from preliminary design information.

\section{Induced Drag}

Calculation of the Oswald efficiency and spanwise loading distribution for the aircraft are performed using an extended vortex lattice method implemented by Mark Drela with the MIT Aero and Astro Department. Athena Vortex Lattice (AVL) evaluates the aerodynamic properties of some aircraft geometry which may be defined with both lifting surfaces and slender bodies like engine nacelles, fuselage pods, etc [38]. Figure 17 shows a general wing geometry in AVL with the spanwise and chordwise loading distribution.

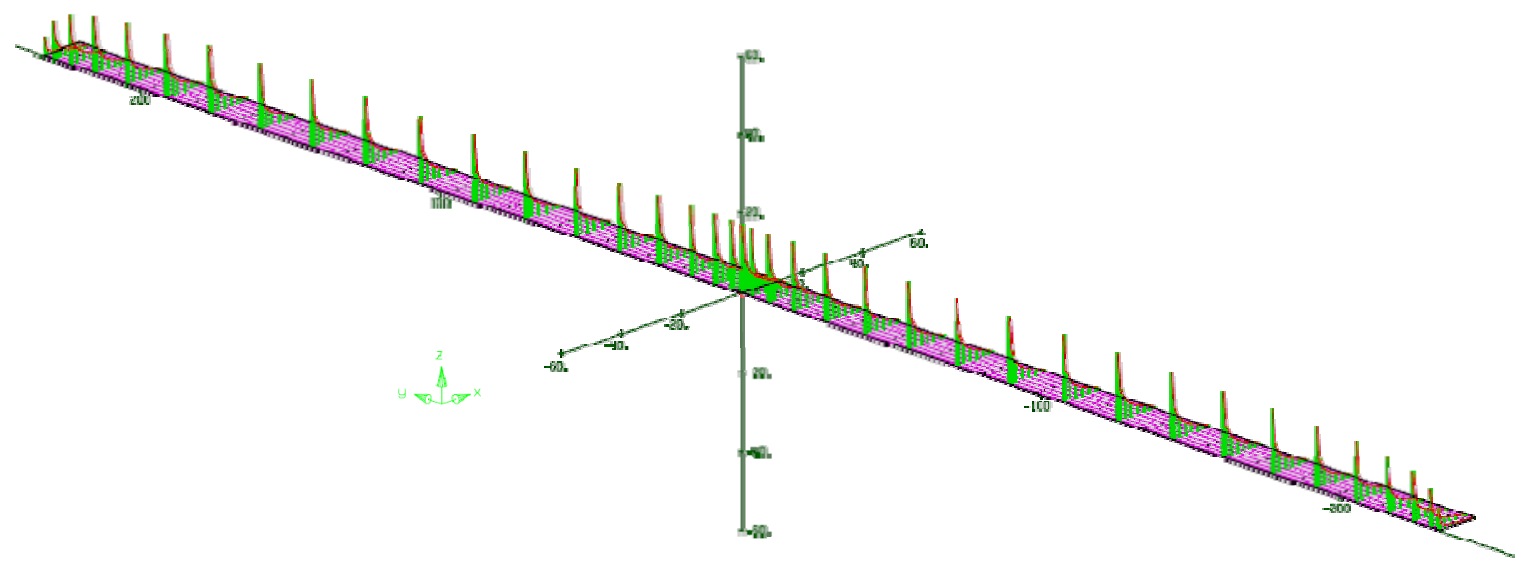

Figure 17 - General Wing Modeled in AVL With Loading Distribution 
This spanwise lift distribution is used to estimate the span efficiency, or Oswald efficiency for induced drag calculations.

\section{Parasite Drag}

Developed by Lockheed Martin for use with rapid conceptual design, EDET, an Empirical Drag Estimation Technique is employed for this design environment [39]. The method is uniquely capable of predicting design and off-design drag levels of advanced airfoils, and is particularly useful when investigating the trade-off of design parameters like aspect ratio, thickness, and body performance [39]. EDET was initially intended for use with fighter/attack/trainer aircraft, and may seem a poor choice for developing the drag polar of our electric HALE aircraft. Where this intended aircraft genre shows up inside the method is when calculating design Mach number, which then determines applicable ranges of speeds for calculation, and contributes to decisions regarding buffet, compressibility, and pressure drag. In order to reduce the influence of these other types of aircraft on the output, the Fortran source code was modified to consider only the set of Mach numbers applicable to the HALE flight envelope.

Skin friction drag coefficients are computed for each component and for the total configuration based on input surface wetted areas and fineness ratios. Also, the incremental change in skin friction drag for flight conditions off of the nominal Mach number and altitude is computed from wing geometry inputs and a consideration of laminar to turbulent boundary layer transition points [39]. This $\Delta \mathrm{C}_{\mathrm{D}}$ method to predict 
off-design drag values allows the user to run the analysis code only once for a given geometry and perform table lookups for subsequent queries.

The form factor method for drag buildup that is used within EDET is described in [40] and generally takes the form of equation 2 .

$$
C_{D_{F}}=\sum_{0}^{N} C_{F}\left(\frac{S_{w e t}}{S_{\text {ref }}}\right) F \cdot F \cdot\left(\frac{C_{F}}{C_{F_{I N C}}}\right)
$$

Here, $C_{D_{F}}$ is the flat plate friction coefficient and $\left(\frac{C_{F}}{C_{F_{I N C}}}\right)$ accounts for compressibility effects.

For most configurations, there exists an additional level of miscellaneous friction drag associated with items like antennas or surface gaps which are not precisely specified during preliminary design. An accepted method of accounting for additional drag is to include a percentage increase in computed drag as a buffer. Reference [40] describes the process of determining a correlation factor to estimate $C_{D_{F} \text { (actual) }}$ from $C_{D_{F} \text { (computed) }}$. The result from a sampling of aircraft is that computed friction drag coefficient should be increased by $28.4 \%$ to approximate operational friction drag.

Overall, several issues have presented themselves concerning the use of EDET to approximate the drag characteristics of an electric HALE UAV. Specifically, the methods used to develop EDET's drag model were based on aircraft dissimilar to our platform, and may taint our design environment. In spite of this, we have kept EDET for several reasons. First, the EDET source has been slightly modified to discourage the interpretation of ours as a supersonic design and control output lookup tables to applicable Mach numbers. Second, if the method incurs error, it is reasonable to assume 
that it will over predict drag rather than under predict the drag of our aircraft. The correction factor of $28 \%$ to account for miscellaneous drag was developed in [40] from a collection of test data for fighter, attack, and trainer aircraft which exhibit many more contributors to miscellaneous drag than a carefully constructed HALE UAV would. Lastly, and most importantly, the central focus of the work contained herein is to develop a modular multidisciplinary environment for aircraft analysis, design, and optimization that allows the user to parametrically study the effect of changing configuration or mission parameters. The goal of such parametric studies is to evaluate potential benefits of one configuration over another, and as long as the drag model is sensitive to these changes in configuration, it will suffice. However, if it is determined that a drag model more specific to HALE UAV's is desired, the modular nature of the environment allows easy replacement of the EDET routine.

\section{Drag Polar Utilization}

It is necessary, at this point, to clarify the operation of our aerodynamic analysis as it relates to an integrated design tool. While difficult to accurately represent all functions of aerodynamic analysis in one single module of a system-level HALE MDA environment such as Figure 14 or Figure 40, specifying two categories provides clarity. The first of which represents the drag polar and the second deals with aerodynamic loads. Rather than describing the drag polar on the same plane as something like Mission Analysis, it is more appropriate to refer to it as a subroutine frequently accessed by other analysis modules. In this way, the drag polar is more analogous to a standard atmosphere 
model than to things like Structures or Energy Storage. However, an Aerodynamic Loads module is employed on the top level, and deals mainly with communicating load distribution information to other disciplines.

Operation of the drag polar and associated aerodynamic analysis happens as follows. When a new configuration is specified for analysis, a geometry module estimates wetted areas and fineness ratios, and distributes associated configuration information where appropriate. MATLAB generates an input file for AVL and performs the vortexlattice analysis, generating spanwise loading information and the wing efficiency factor, e. The span efficiency is included in the generation of an EDET input file, and again MATLAB issues a system command, this time to run EDET as the external application. EDET performs a parasite drag analysis and incorporates both the span efficiency and a pressure drag term into the final drag polar. Drag polar information is relayed to the rest of the MDA environment as a set of table lookups, one finds $C_{D}$ for combinations of Mach and $\mathrm{C}_{\mathrm{L}}$, and the other relays the incremental $\Delta \mathrm{C}_{\mathrm{D}}$ from Mach and altitude.

Here, an example is given for an aircraft based on Helios (Figure 18). This configuration is passed to a code routine that creates formatted text files for AVL and EDET, generates run scripts, and saves formatted output files.

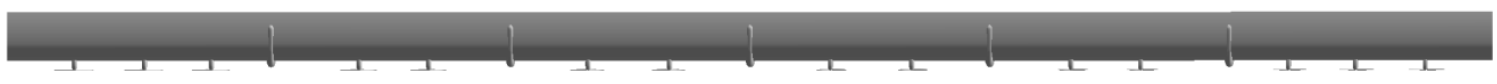

Figure 18 - General Baseline Geometry

As described above, automated post-processing of the EDET output file generates lookup tables for a space of Mach numbers, altitudes, and $\mathrm{C}_{\mathrm{L}}$ 's. Drag polars for two flight 
conditions are shown in Figure 19, one for takeoff and one at the top of climb, arriving at 65,000 feet. Immediately noticeable is that the parasite, or zero-lift drag coefficient has increased with altitude.

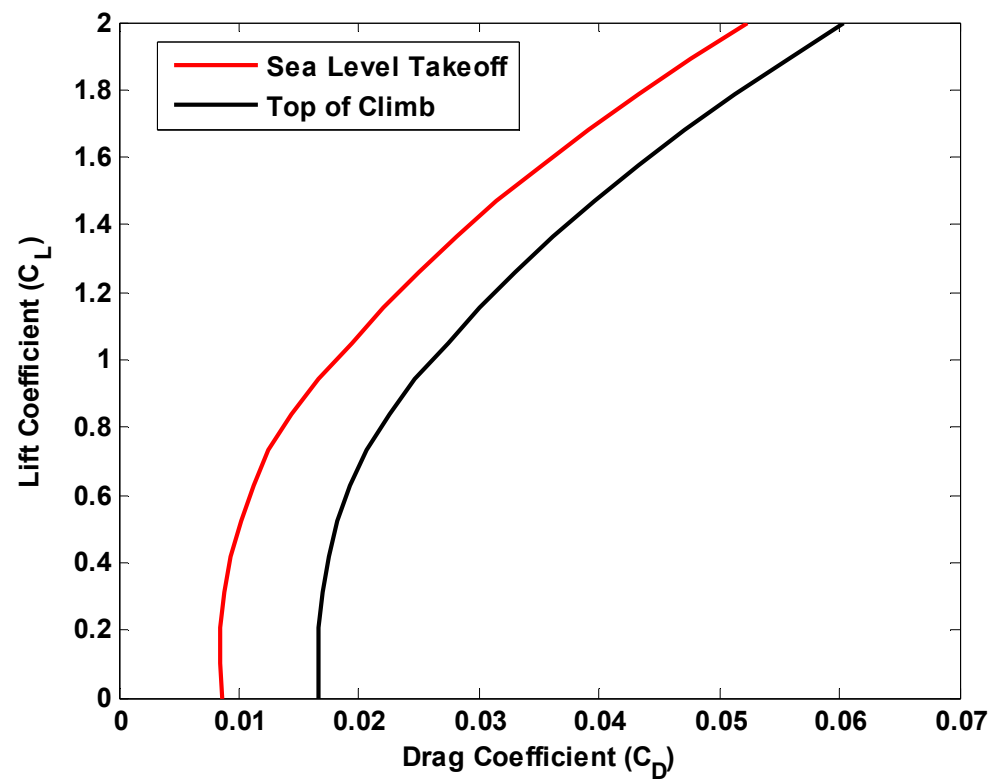

Figure 19 - Drag Polars During the Helios Baseline Analysis

Another important piece of data involved with the drag analysis is the Reynolds' number. Figure 20 illustrates the actual Reynolds' number for the Helios-style aircraft during the climb segment of its mission. Partway through the climb, there is a change in Reynolds' number contours caused by a change in atmospheric levels in the standard atmosphere. 


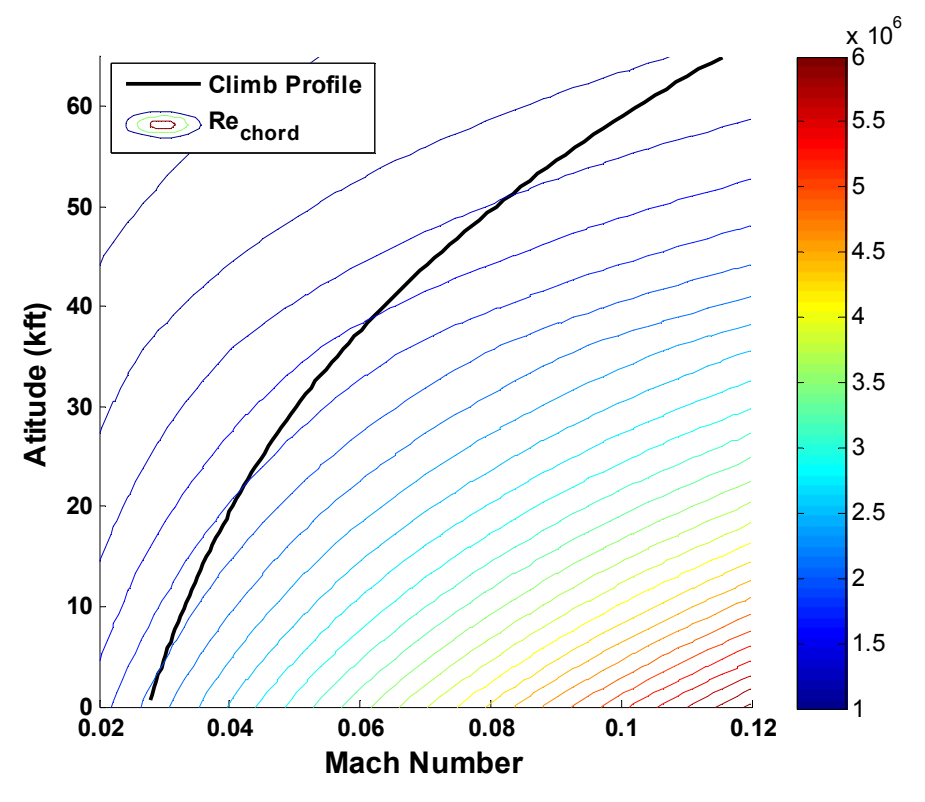

Figure 20 - Reynolds' Number Based on Chord

Determining the contributors to parasite drag is also helpful in understanding the analysis module. An aircraft configuration like Helios HP01 or the general case at hand (Figure 18) has a small number of contributors to parasite drag. Really, besides the wing, there are only the battery/payload pods. However, the EDET routine also assigns miscellaneous friction drag at nearly $30 \%$ of the total (per the earlier discussion), so the parasite drag buildup for our baseline case is dominated largely by the wing contribution. Figure 21 shows the cruise parasite drag contributions and list the drag counts associated with each, corresponding to the $\mathrm{C}_{\mathrm{Do}}$ of 0.017 from the Figure 19 top of climb drag polar.

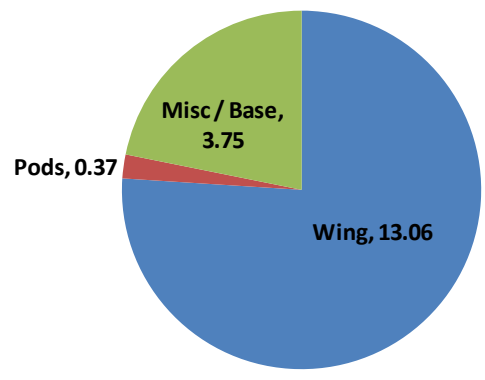

Figure 21 - Parasite Drag Breakdown, Component Drag Counts 
Now, we must also ensure that the drag module produces correct trends for changing aspect ratio. Examples up to this point have been repeated for wings with aspect ratios of 30,35, and 40, resulting in the cruise drag polars shown in Figure 22. Note that the drag polars of Figure 22 do not necessarily represent closed designs, but rather are intended to illustrate the reaction of the drag analysis code to aspect ratio. For these three cases, wing reference area is assumed to be constant. Resulting trends do follow expected behavior, reducing induced drag and increasing $\mathrm{L} / \mathrm{D}$ with increased aspect ratio.

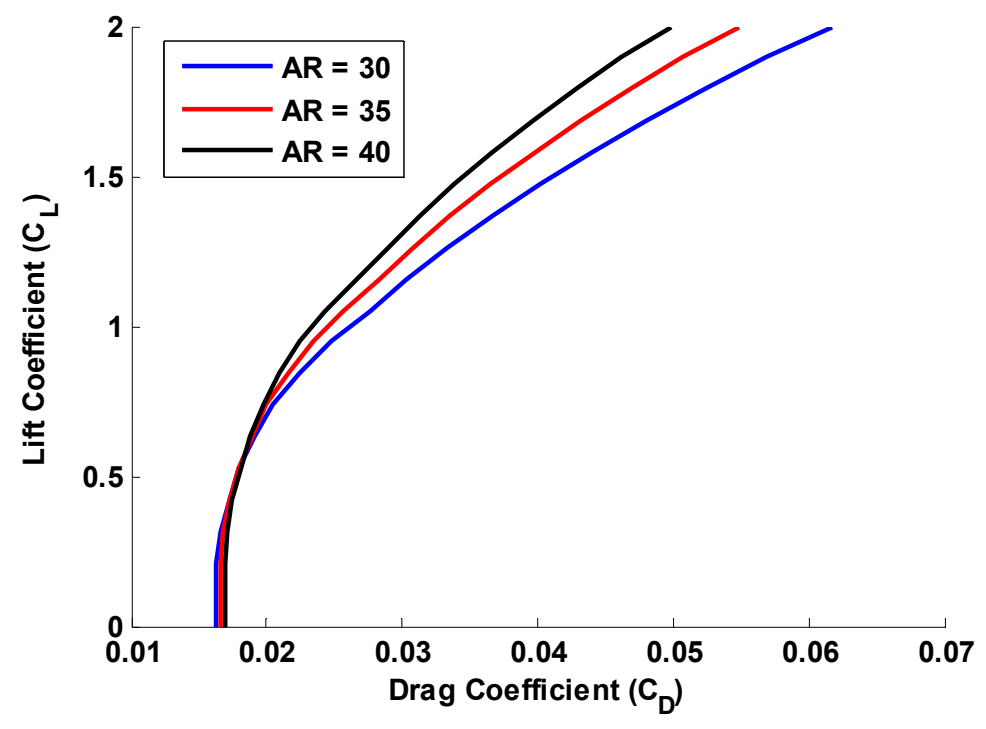

Figure 22 - Effect of Aspect Ratio on Drag

Finally, the handling of laminar to turbulent transition in the EDET source code shows some interesting trends for lift to drag ratio $(\mathrm{L} / \mathrm{D})$. It is apparent that there are certain flight regimes at low speeds or high altitudes where the flow is assumed to be laminar in the EDET analysis. In these locations, the L/D ratio of the aircraft is increased. 
For the general Helios aircraft in Figure 18, the max L/D jumps from 34 or 35 up to 45 or even as high as 50 in some locations. Figure 23 shows a surface plot of L/D and the red areas where L/D increases indicates EDET assuming laminar flow, the plot is cut off on the left because of EDET's limitations on building $\mathrm{C}_{\mathrm{L}}$ lookup tables.

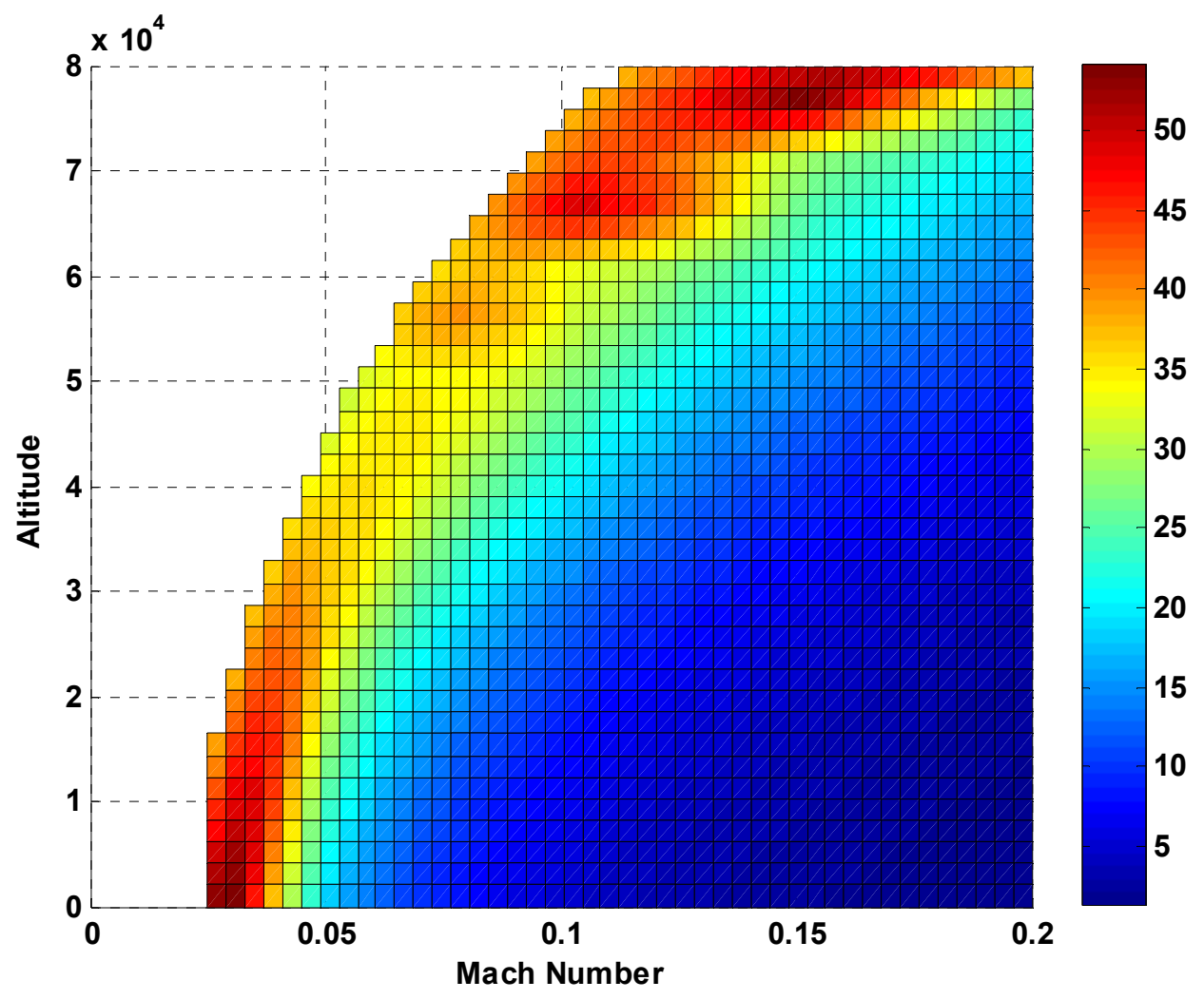

Figure 23 - Lift to Drag Ratio

With an understanding of the fundamental behaviors of the aerodynamics module, we must still decide how good the results are. For this we turn to an aircraft with known and available data, and the following section describes the validation effort for this discipline. 


\section{Drag Polar Validation}

In order to evaluate the drag polar and aerodynamic analysis developed for this tool, the process is applied to an aircraft with known performance. Here, the Virgin Atlantic GlobalFlyer is used as a test case. Figure 24 shows the aircraft modeled in the Vehicle Sketch Pad environment, which provides wetted area and geometrical data.

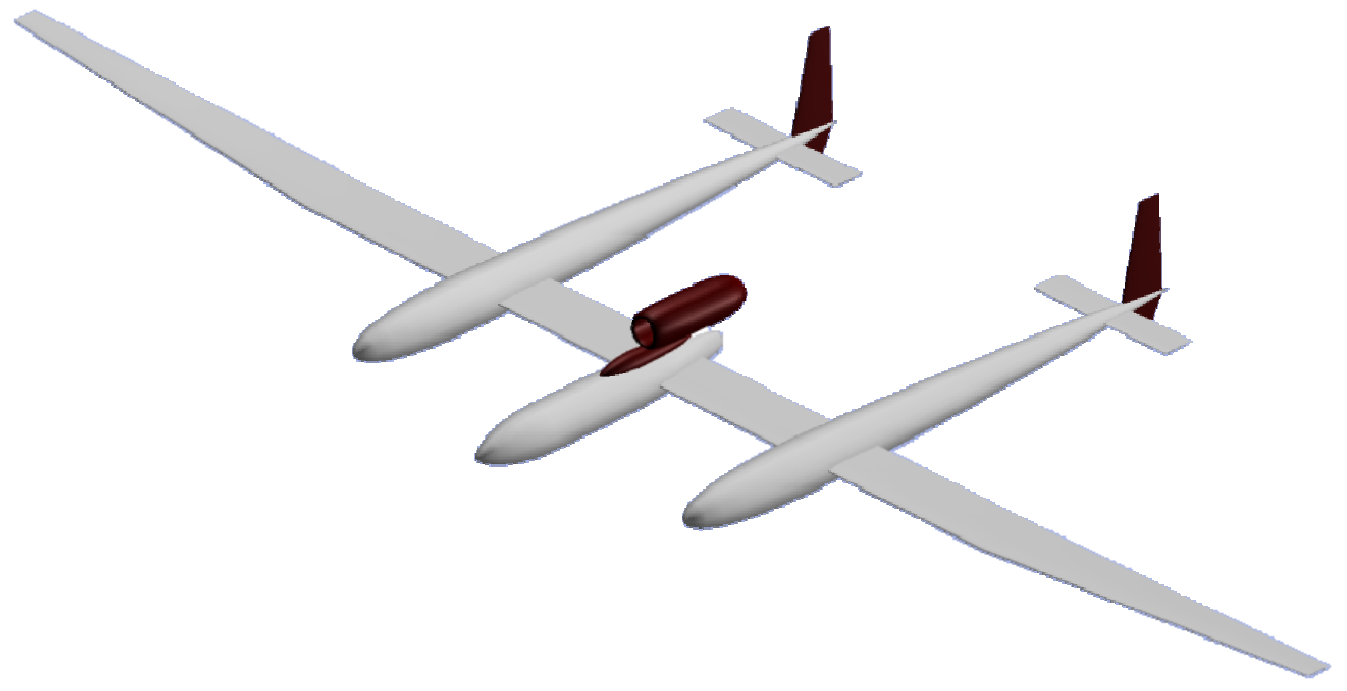

Figure 24 - GlobalFlyer Modeled in VSP for Drag Validation

Of the available data for this aircraft, the one characteristic that applies most to a drag analysis is the maximum lift to drag ratio, quoted at 32 by Steve Fossett in an interview presented in [41]. The geometry of Figure 24 is evaluated in EDET and the resulting drag polar for cruise at 51,000 ft is shown in Figure 25. Here, a $C_{\mathrm{Lmax}}$ of 2 was assumed as the upper cutoff, and a cruise Mach number of 0.28 is used. GlobalFlyer has an aspect ratio of 32.5 and a maximum wing loading of 56.5 when full [42]. 


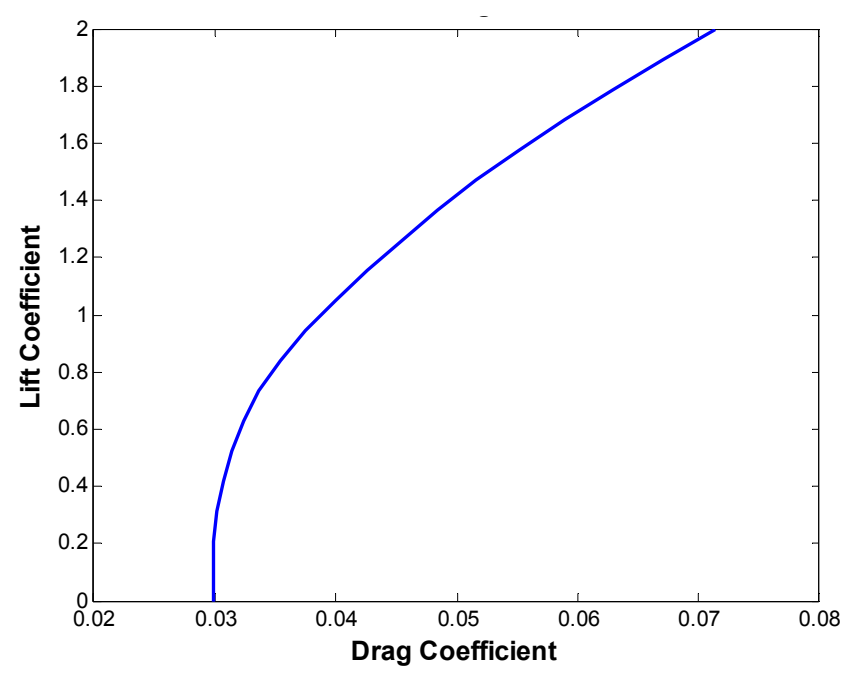

Figure 25 - Cruise Drag Polar for GlobalFlyer

Parasite drag coefficient of just under 0.03 during cruise may also be described more specifically with a component parasite drag breakdown (Figure 26). Parasite drag contributions in the figure are listed by drag count. As noted previously, the miscellaneous drag allowance of $28 \%$ is included.

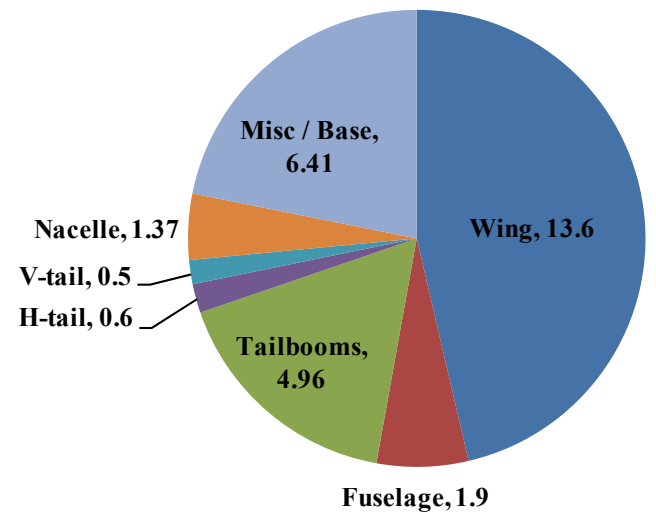

Figure 26 - Parasite Drag Buildup for GlobalFlyer, Component Drag Counts

From the approximation of the Virgin GlobalFlyer drag polar, we find an estimated maximum L/D of 32.04 at $51,000 \mathrm{ft}$. Though we have made some assumptions about the aircraft, like $\mathrm{C}_{\mathrm{Lmax}}$, the best glide ratio is definitely in the realm of acceptable values and reflects favorably on the drag and aerodynamic analysis processes included. 
One final area of aerodynamic analysis we must examine is the sensitivity to wing thickness. As mentioned in the optimization overview, thickness-to-chord ratio is one of the design variables for optimizations included in this study. Later, when discussing the structures module and results, we see that the thickness of the wing has a drastic effect on aircraft structural weight. It is important, then, that we correctly model penalties associated with thick airfoil sections so that the optimization environment realizes the trade between thick wings that have higher drag but are structurally favorable and thin ones that result in low power requirements but heavy spars. For the GlobalFlyer validation case, the wing thickness assumed to be $11 \%$ as the coordinates for the Roncz CAP15 airfoil are unavailable. Changing the airfoil thickness-to-chord ratio to $30 \%$ when calling EDET results in the drag polar shown as a red line in Figure 27. Analysis of the parasite drag breakdown of the new configuration shows that only the wing and miscellaneous drag counts have increased, raising the parasite drag coefficient by $19.4 \%$ and decreasing the max L/D to 29.5. This example confirms that the aerodynamic analysis exhibits sensitivities where necessary and produces acceptable drag results.

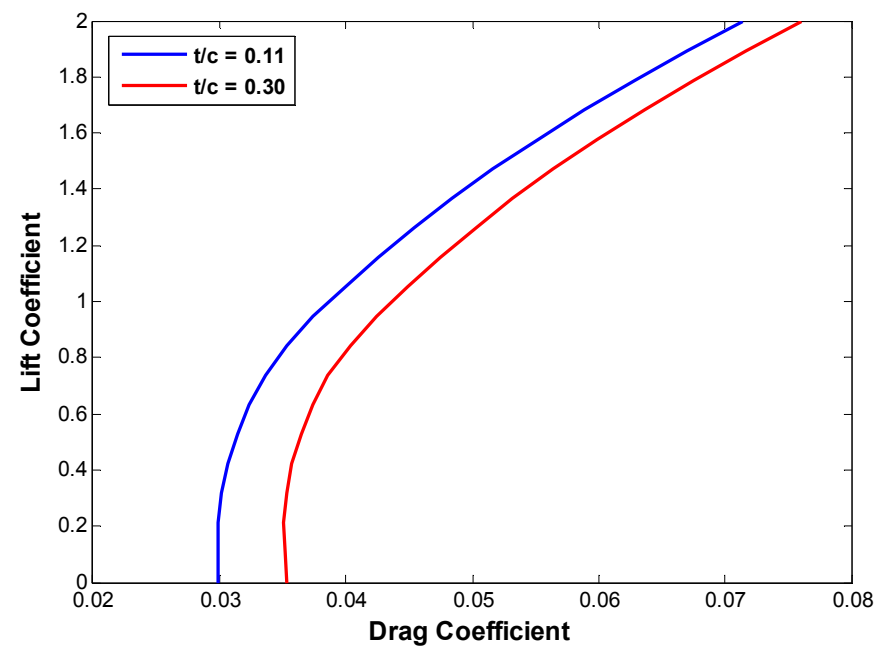

Figure 27 - Effect of Wing Thickness on Drag 


\section{Electrical Energy Modules}

When discussing constraints imposed on the optimization, energy generation, use, and storage for the aircraft related to one of the fundamental requirements. Long endurance is taken to mean repeatable 24 hour operation for periods anywhere from days to years as specified by DARPA for Vulture. As with any aircraft system, the designer, or in this case the automated design environment, must have an understanding of where propulsive energy comes from and where its sensitivities lie. The two main categories of our energy system are energy generation and energy storage, and are connected through analysis of daily energy required and available. However, energy system weights cannot be developed without analyzing the propulsion system and power train efficiency. As such, this section will continue with a description of the propulsion module and the method for energy system weight estimation.

\section{Solar Energy Generation}

The first step in evaluating solar energy generation is to model the total energy available from the sun at the estimated flight conditions of the aircraft. A good discussion of solar irradiance is given in [43] by Duffie and Beckman, but their model of hourly solar energy involves parameters like hour angle of sunset that may complicate its application by someone less well-versed in solar terminology. To simplify and generalize the model, hourly solar irradiance may be modeled by a sinusoidal function (3).

$$
I_{(t)}=\left\{\begin{array}{cc}
I_{\max } \sin \left(\frac{\pi\left(t-t_{\text {sunrise }}\right)}{t_{\text {daylight }}}\right) & \text { day } \\
0 & \text { night }
\end{array}\right.
$$


Reference [44] shows that a sinusoidal function like this closely follows the application of the rigorous model of Duffie and Beckman. With maximum irradiance and daylight timelines as inputs, modeling alternative latitudes, seasons, or weather conditions is easily accomplished. Maps of solar power available for different months across various latitudes are available in [14].

After modeling the delivered total energy from the sun, we must examine how much energy the aircraft can actually harvest. Several parameters in the MDA environment determine how much energy will be available. Equation 4 describes the available solar energy from the panels, where $\eta_{\text {sol }}$ is the efficiency of the individual solar cells, and $\kappa_{\text {sol_coverage }}$ is the percent coverage of the wing area.

$$
E_{\text {sol }}=\eta_{\text {sol }} \kappa_{\text {sol_coverage }} I_{(t)}
$$

Taking into account both the total solar energy provided and the efficiency of our collector, a model of total available solar power is developed. Several resources are used to center our model; Figure 28 shows the reference solar models alongside those employed in this MDA environment. 


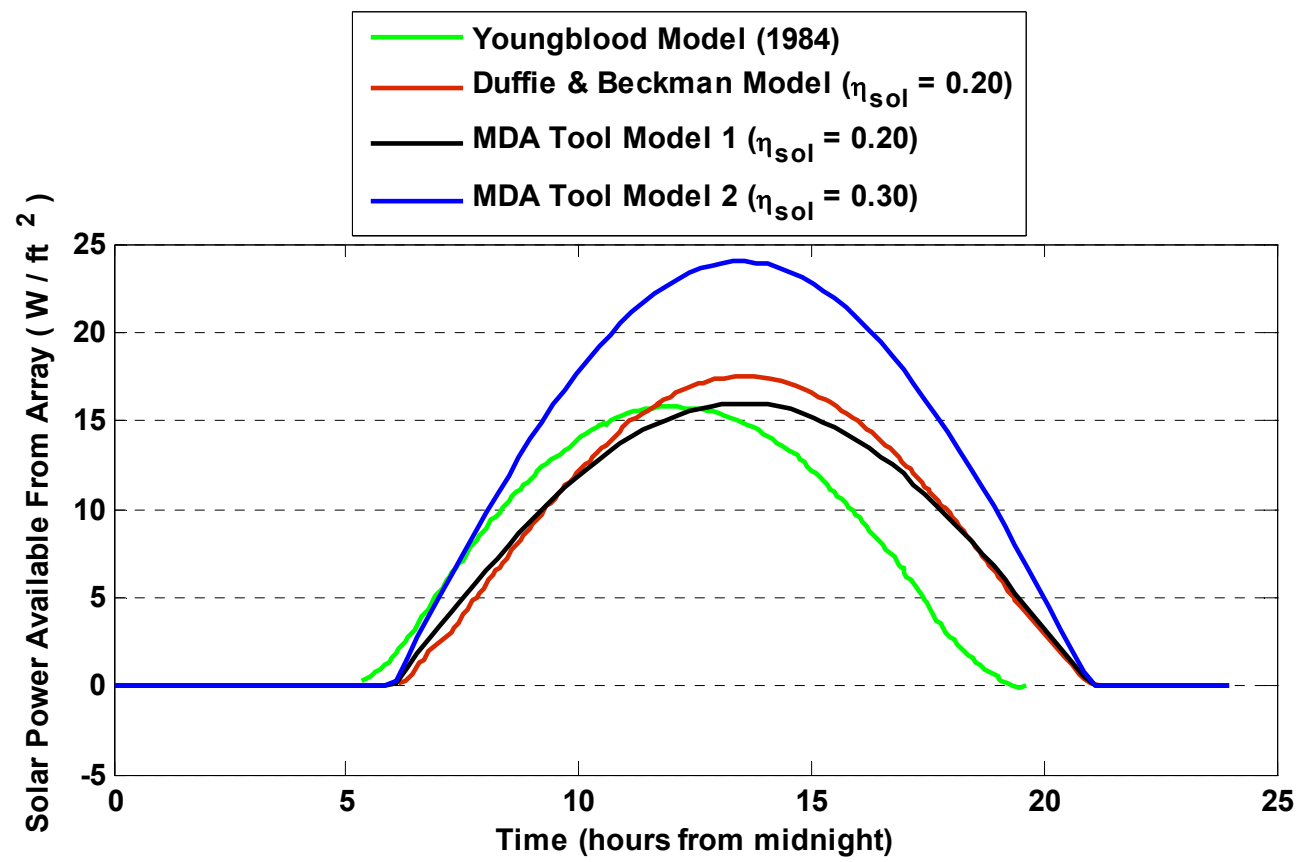

Figure 28 - Basis for Available Solar Energy Models [14], [36], [37]

The main difference between the reference models in Figure 28 is the length of day. Youngblood's model represents a day in July at a latitude $31^{\circ}$ North where the Duffie and Beckman model (adapted by Noth [44]) is defined parametrically for Lausanne, Switzerland at $46^{\circ}$ North, and may vary year round. The model selected for our analysis is based on Duffie and Beckman, with an allowance for varying solar cell efficiency as an indicator of technology.

\section{Energy Storage}

Achieving the continuous day/night operation desired for a next-generation HALE aircraft hinges on the ability to store enough energy during daytime flight to power the system until the next sunrise. Several fundamentally different approaches have been investigated for storing energy, namely chemical energy or gravitational potential 
energy. A good method for considering a variety of chemical energy storage technologies is presented in [36] where energy requirements and weight models are developed based on the energy density (Watt-hr/kg) of the technology. This method allows us to model different technologies like fuel cells or batteries simply by changing the energy storage weight factor. The second approach to storing energy is to use excess power from solar cells to gain altitude, and when solar energy is no longer available, the aircraft would enter either a glide phase or an under-powered descent. Solar Impulse is an example of an aircraft which utilizes both energy storage methods to achieve a sustainable daily energy cycle. Once the aircraft batteries are fully charged during a level flight, the aircraft increases the power to the engines to the maximum available and climbs until sunset. Night flight then begins as an underpowered descent until the initial altitude is reached, where batteries increase power to maintain level flight [45].

The current energy storage model included in this study accounts only for chemical energy storage, but may implement either batteries or a variety of fuel cells. Coupling the flight planning and mission analysis to the energy analysis to allow for gravitational potential energy storage is possible for future revisions.

For the study at hand, advanced batteries are considered as the storage medium, but an input flag allows the user to consider Proton Exchange Membrane (PEM) fuel cells powered by either liquid or gaseous Hydrogen. Metrics for component weights of energy storage items are defined in [36] and account for whatever tanks or fuel may be necessary, as well as a power management and distribution unit. 


\section{Daily Energy Model}

The final step in analyzing the energy system of our HALE aircraft is to integrate the energy generation and storage routines with the power requirement outputs from the mission analysis module. Figure 29 shows a multiple day cycle of the aircraft energy processes. Adjustments for day 1 are necessary to account for takeoff and climb, as well as the cruise leg of the mission to reach the desired loiter location. Tying the model for solar power discussed earlier together with mission power requirements including payload operation and flight systems power draw produces the profile of extra energy in Figure 29. This extra energy is simply the difference between the total amount produced by the solar array and what is needed for flight and mission operations. Extra power then may be used to support energy storage systems.
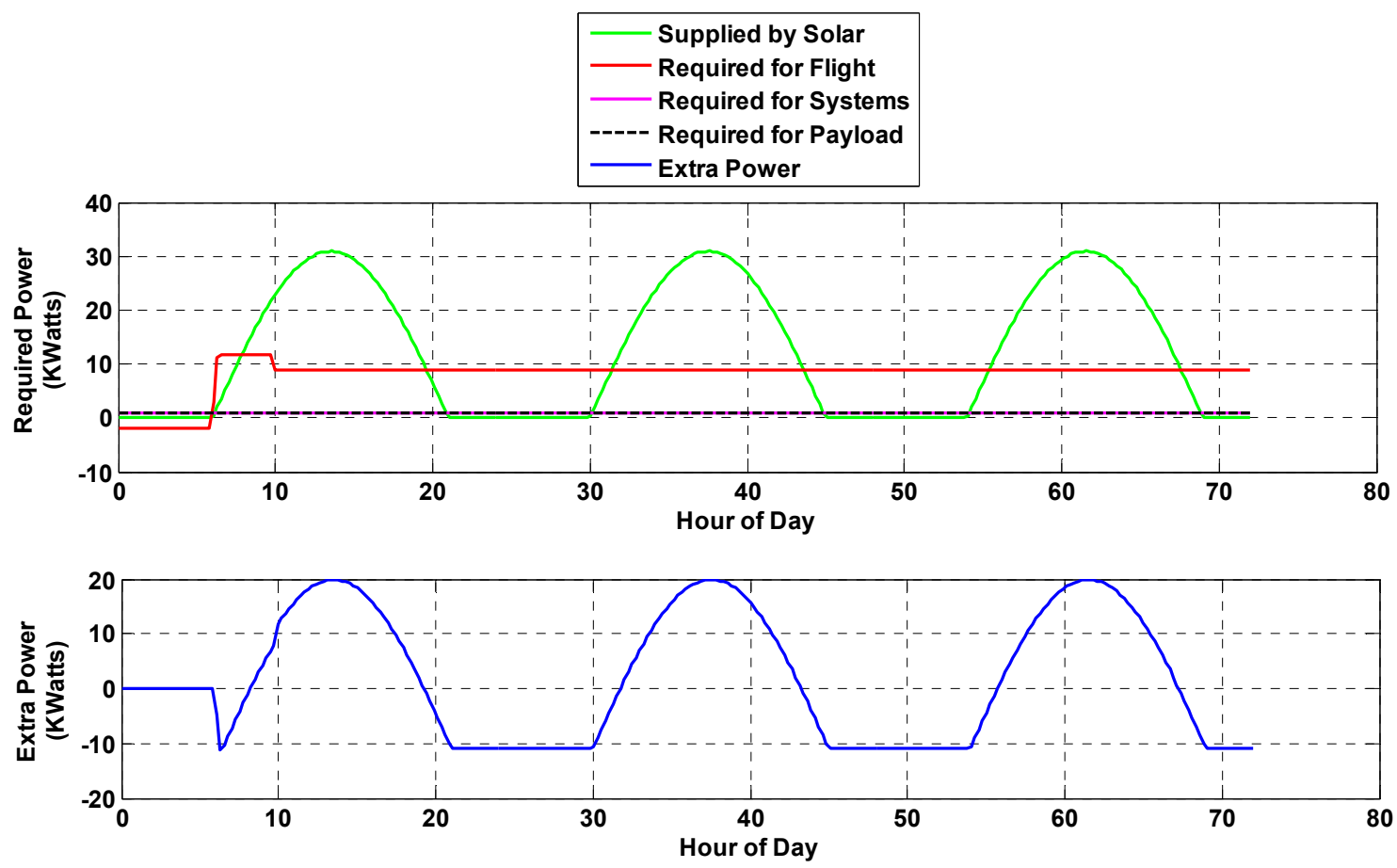

Figure 29 - Model for Available and Required Power (above) and Resulting Extra Power (below) 
Charge delivered to the batteries is calculated from integrating the extra power over time, and will generally result in a battery charge profile like Figure 30. Keep in mind that Figure 30 represents a prefect energy storage medium with no losses due to cycling.

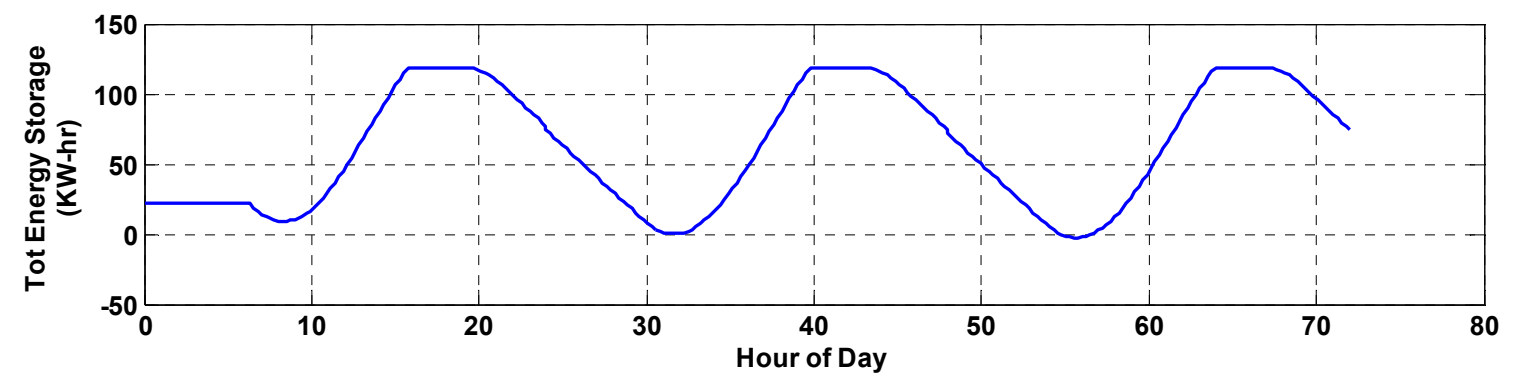

Figure 30 - Energy Storage Charge Profile

The plateau of battery energy that exists for certain configurations, including the example case presented here, is the upper bound of battery energy needed to sustain nighttime flight. Between the energy generation and storage subroutines, the power required for flight is integrated for time where solar power does not suffice, resulting in the total energy storage requirement. Included in the storage requirement are the times when solar power is available but it is less than what is required for flight, depicted in Figure 29. Lastly, it is important to reiterate that the required power for operation includes power necessary for flight as well as that required for payload operation and an allowance for aircraft systems power requirements. 


\section{Propulsion}

In order to discuss the propulsion aspects of our model, it is important to be clear about the overall MDA/MDO process. Power to weight ratio in Watts per pound is specified as an input to the analysis, and specifically represents the power delivered to the propeller during flight. For this reason, we must include the propeller efficiency, $\eta_{\mathrm{p}}$, in any analysis concerning thrust or power, and frequently use the expression

$$
T=\left(\frac{P}{V}\right) \eta_{P} \text { or } \frac{T}{W}=\left(\frac{P}{W}\right)\left(\frac{\eta_{P}}{V}\right)
$$

A general schematic of an electric propulsion system is shown in Figure 31. When evaluating the energy requirements, estimations of efficiencies for each component in the power system are provided. Also, Figure 31 shows the different expressions for power at different locations in the system, changing from mechanical thrust power at the propeller to shaft power at the prop and shaft power at the motor, $\mathrm{Q}$ represents torque and $\mathrm{n}$ is the rotational speed of the shaft. Lastly, the power is expressed electronically as the product of current and voltage supplied by the speed controller.

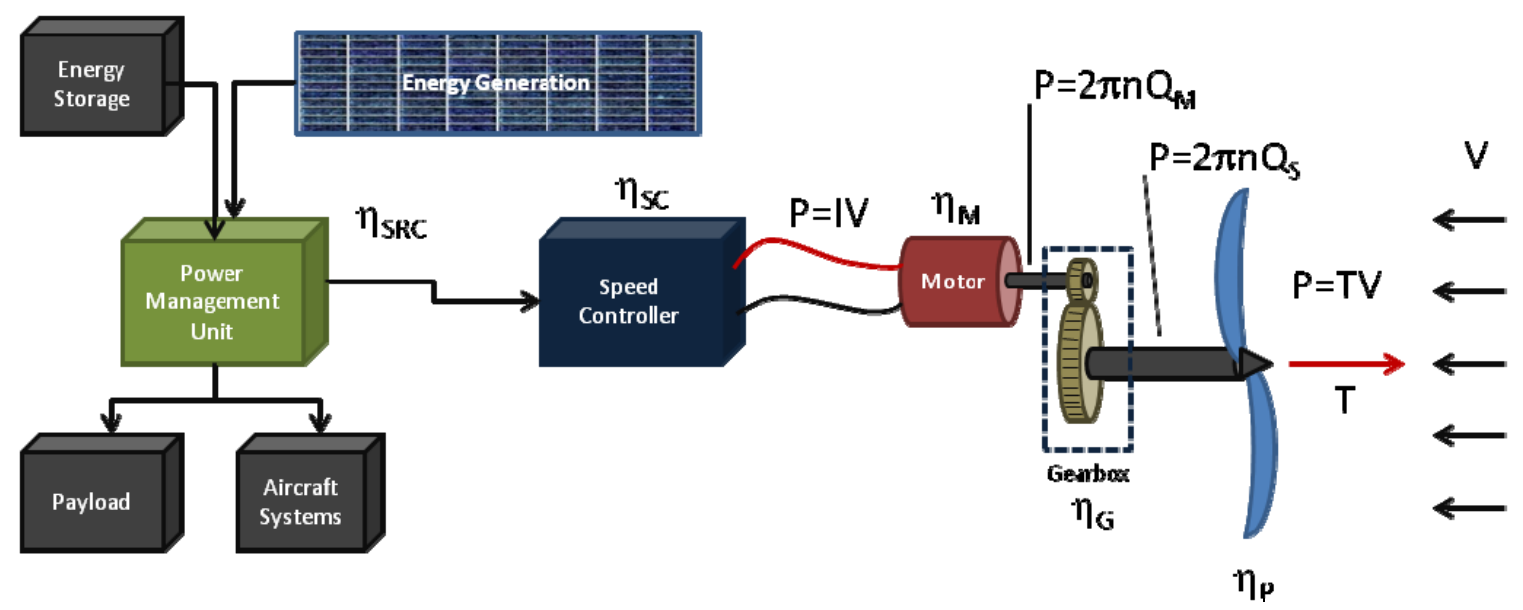

Figure 31 - HALE UAV Power Systems 
A description of the efficiencies is presented in Table 7, along with some assumed values for the components, intended to be conservative.

Table 7 - General Power Train Efficiencies

\begin{tabular}{|c|l|l|}
\hline Symbol & Description & Value \\
\hline$\eta_{P}$ & Propeller efficiency & 0.80 \\
\hline$\eta_{G}$ & Gearbox efficiency & 0.95 \\
\hline$\eta_{M}$ & Motor efficiency & 0.90 \\
\hline$\eta_{S C}$ & Speed controller efficiency & 0.90 \\
\hline$\eta_{S R C}$ & Source efficiency (batt,fc, etc.) & 0.90 \\
\hline
\end{tabular}

Using a more flexible sizing method like the one given in [36] requires the definition of several additional efficiency parameters. Also, more specific data is available for several items in Table 7. Just as an example, the source efficiency in Table 7 of $90 \%$ may be reasonable, or even conservative for batteries, but if we decide to implement fuel cells, the source efficiency drops to only $50 \%$.

Table 8 - Specific Power Train Efficiencies [36]

\begin{tabular}{|c|l|l|}
\hline Symbol & Description & Value \\
\hline$\eta_{B A T T}$ & Battery efficiency & 0.98 \\
\hline$\eta_{F C}$ & Fuel cell efficiency & 0.50 \\
\hline$\eta_{P M D}$ & $\begin{array}{l}\text { Power management and } \\
\text { distribution efficiency }\end{array}$ & 0.97 \\
\hline$\eta_{M}$ & Motor efficiency & 0.97 \\
\hline
\end{tabular}

Total power train efficiency is then calculated as the product of applicable component efficiencies for the power system employed. There is a subtlety, however, pertaining to energy processes within the MDA environment. As pointed out on the 
previous page, when interested in the thrust available from the aircraft, a propeller efficiency is already built into the equation. Consequently, the power requirement from other modules like Mission Analysis describes what must be delivered to the propeller. It follows that integrating that power over the mission time produces the energy required at the propeller, $E_{R}$. What this means is that the energy delivered by the Power Management and Distribution (PMD) unit must exceed this value to account for power train imperfections. Here we designate the energy output from the PMD as $E_{S}$, or the required total stored energy, given in (6)

$$
E_{S}=\frac{E_{R}}{\prod \eta_{n}}
$$

where $\eta_{\mathrm{n}}$ is the efficiency of an energy component $\mathrm{n}$ in the power train, but does not include propeller efficiency.

With regard to propeller efficiency, Table 7 lists it as a static value despite the fact that for fixed-pitch propellers, as many HALE UAV platforms may well be, the efficiency changes during flight. There are several reasons that this parameter $\left(\eta_{\mathrm{p}}\right)$ has been implemented as such. First, considering the preliminary stages of design we are operating in, propulsive sizing requires a scalable architecture. This means that rather than selecting a specific propeller, we assume that if we use a reasonable efficiency value then eventually a prop may be found to meet or exceed it. Second, the primary focus of this effort is to investigate parametric changes and sensitivities. Accordingly, it is valid to use a reasonably assumed value for $\eta_{\mathrm{p}}$ as long as we evaluate how changing the assumption affects the resulting aircraft. This sensitivity study is presented in the Tool Validation section. 


\section{Energy System Weights}

With both energy system efficiencies and data from Mission Analysis, the MDA environment has developed the total system energy storage requirement, $E_{S}$, for a mission. The final task for the energy and propulsions module, then, is to determine how much the energy system must weigh. Energy system components can be broken up into three categories. First, energy generation describes things like solar cells and supporting infrastructure. Second, energy storage represents batteries, or fuel / fuel tanks if applicable. Finally, energy conversion components are those that transform stored energy into useful work. Energy conversion is where we classify fuel cells, Power Management and Distribution units, and motors.

Weight of the energy generation system, essentially the solar panels and equipment, is estimated based on the required solar coverage area. Information from several sources concerning modern solar cells leads to an area density of the solar power system, $\rho_{\text {Asol }}=0.11$ pounds per square foot of solar area [46], [47].

Energy storage weight is developed from the total energy storage requirement, $E_{S}$, and information regarding the specific energy density of storage components. This technology metric has been discussed previously for batteries, but if fuels are used with a fuel-cell, the sizing methods of [36] provide applicable information, adapting an energy density metric to scalable components like liquid Hydrogen tanks.

Energy conversion weights are built up from power density specifications rather than energy density. For example, [36] claims advanced electric motor technology to be capable of producing $3.4 \mathrm{~kW} / \mathrm{kg}$ of motor weight $(1.54 \mathrm{~kW} / \mathrm{lb})$. So calculating energy conversion system weight involves connecting the total aircraft power requirement with a 
summation of whichever energy conversion components are part of the system. When evaluating Helios baselines, however, we know that each motor provided $1.5 \mathrm{~kW}$ of power [21]. Therefore a general estimate for weight per motor is used based on commercially available brushless DC motors of similar power. Total number of motors may then be estimated from the maximum power per motor, the power to weight ratio of the aircraft, and the gross weight. 


\section{Structures}

Estimating structural weight is another highly important aspect of preliminary design. Several methods for mass prediction are possible, involving varying levels of sensitivity to configuration, validity, or computational simplicity. Standard methods include using historical regressions of existing aircraft data to approximate weights of components or subsystems. Specifically, [33] develops weights for elements of the airframe like the spar, ribs, control surfaces, fuselage, et. al. as functions of the total weight and wing geometry. One example of implementing these weight predictions is given in [48]. For application to the work herein, there are several factors discouraging the use of such methods. First, the methods in [33] were published in 1984, and therefore the platforms that make up the regression models predate several key technology demonstrators or recent design efforts. Also, investigating an unconventional configuration like a pinned-segmented flying wing discourages use of such weight models because it diverges from the aircraft basis for the regression. For this reason, a bottom-up weight model is developed, relying strongly on a structural analysis of the wing geometry.

There are several decisions that must be made prior to completing structural analysis. Fundamentally, the purpose of our structures module is to design the spar cross section to withstand a certain loading condition, and output the resulting spar weight. In addition, the module will estimate a total wing structural weight. These tasks require knowledge of the spar geometry, material properties, and desired factor of safety, as well as loading information. The structural design of our HALE UAV will mimic that of the NASA ERAST vehicles and Helios and is composed of a tubular spar cross section and a 
non-stressed wing skin (Figure 32) [21], [49]. Likewise, wing structural material will be a carbon fiber composite.

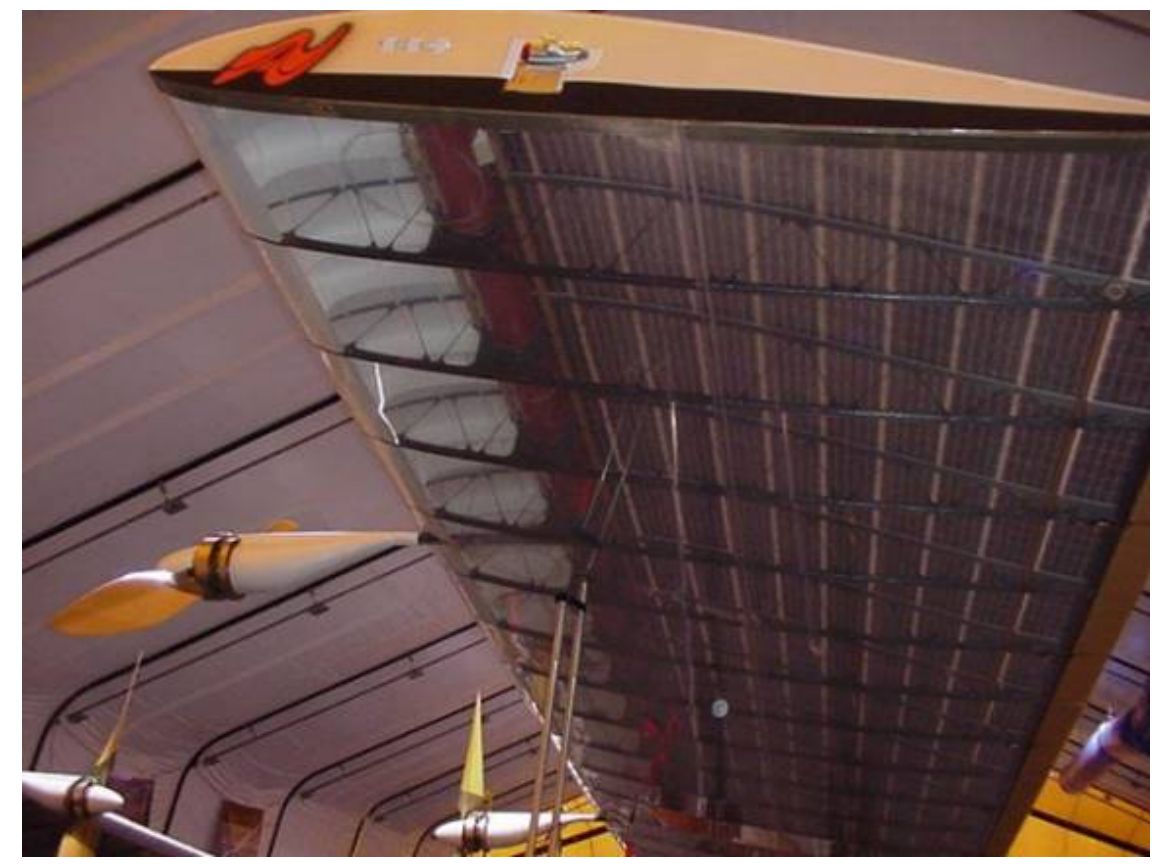

Figure 32 - Helios Wing Structural Arrangement [50]

Figure 33 shows the process for performing the wing structural analysis and developing a weight estimate.

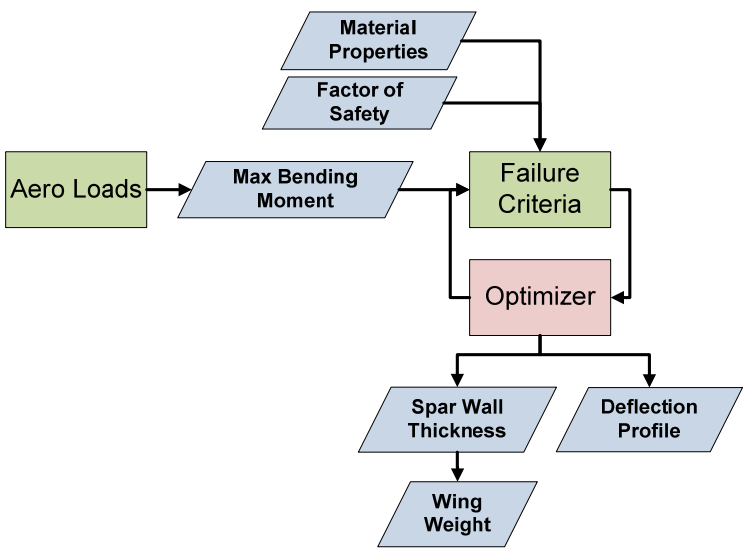

Figure 33 - Structural Analysis Process

First, the aerodynamic loading distribution is brought in from the Aero Loads module, and when combined with the span loaded weights of other systems (motor, solar, energy 
storage, etc..), a net loading distribution is available. Figure 34 shows a general spanwise loading for the baseline single-segment wing configuration, major point loads represent the locations of battery pods. The net loading is then integrated to produce shear and bending moment distributions (Figure 35).

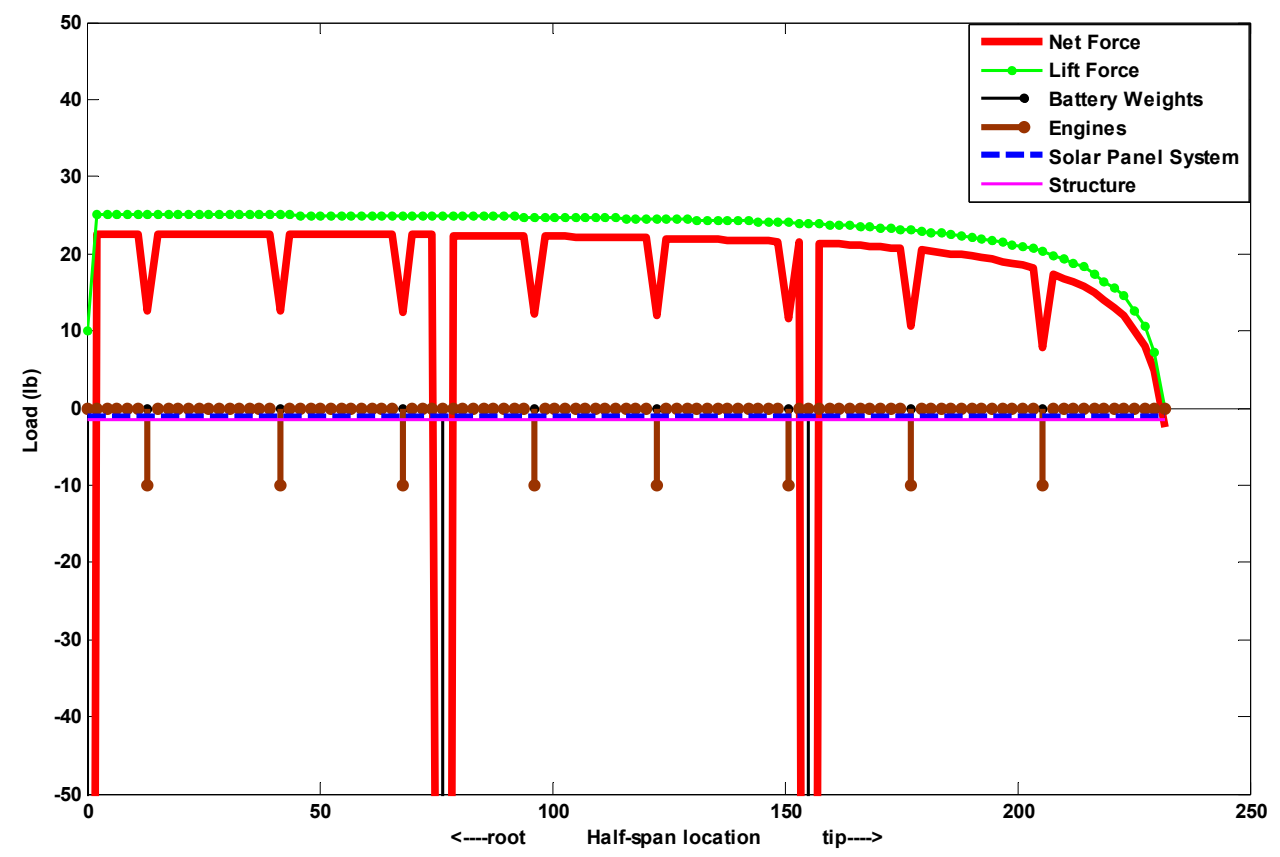

Figure 34 - General Spanwise Loading Distribution
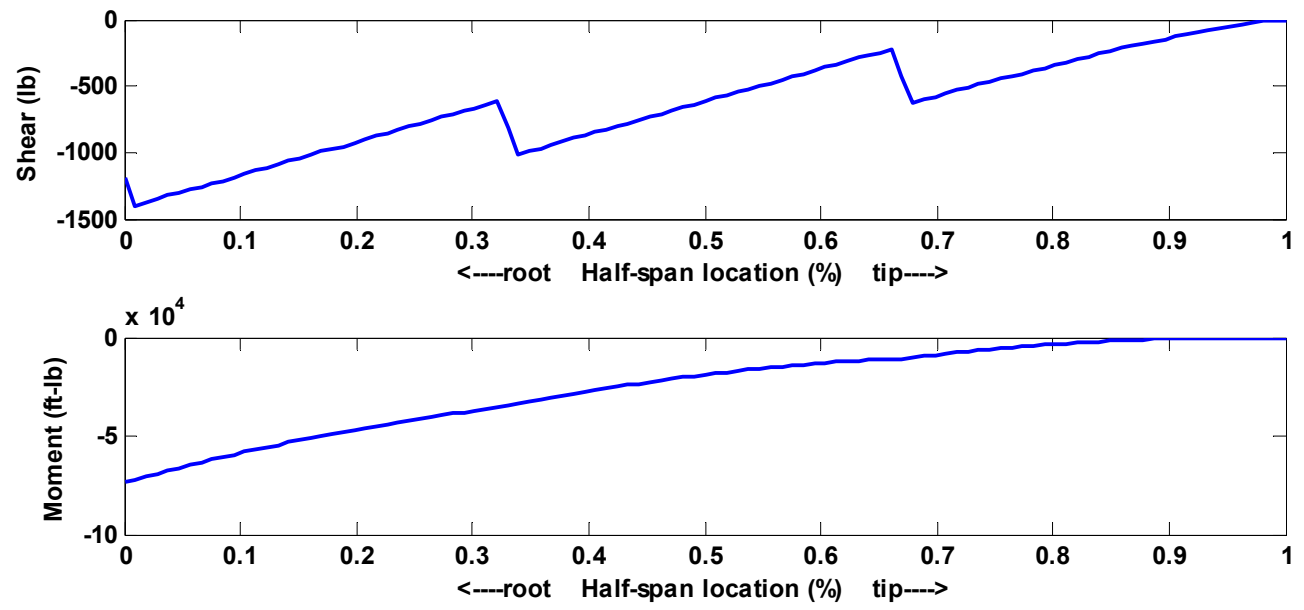

Figure 35 - General Spanwise Shear and Moment Diagrams 
In an attempt to approximate a tapered spar geometry, the design tool divides the semispan of the spar into some number of sections. From the bending moment diagram, the maximum bending moment for each section of spar is stored and an optimization routine determines the minimum wall thickness for each section to satisfy a specified factor of safety (Figure 33). The failure criteria used is the Maximum Stress Theory for composites as described by the Center for Composite Material Research [51]. Material properties used for analysis are based off of a carbon fiber epoxy sheet molding compound designed for military and aerospace structural applications [52]. Figure 36 shows a generic thickness profile along the semispan for a spar divided into ten sections.

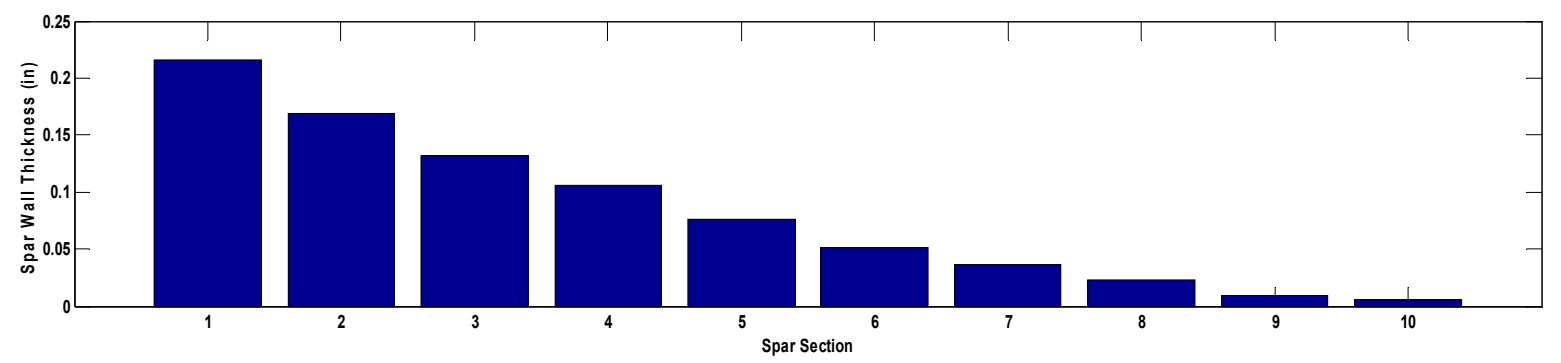

Figure 36 - Spar Wall Thickness Profile

When applied to a configuration of multiple wing segments, the only difference in the process is the interpretation of loads. For aerodynamic loads, an enveloping case is applied to all segments to achieve identical segment design. Also, all point loads for batteries, motors, or payload are distributed evenly among segments.

So far, the process has developed the spar geometry and weight. To estimate the total wing weight, we look to Figure 32 for inspiration, and notice that besides the spar, the only other components are delicate ribs and a polymer wing covering. The upper 
surface of the wing is occupied by solar panels, of which we estimate weight separately. Currently, the total wing weight is estimated at 1.1 times the spar weight because of the ultralight construction methods available.

\section{Weights}

The specifics concerning weight estimation for each contributing discipline have been discussed in their sections above. Total weight, then, is computed as the sum of the wing structure, tail structure (if applicable), energy storage, energy generation, propulsion components, and payload weights. In finalizing weight statements for a variety of HALE configurations, reference [49] mentions a $6 \%$ mounting and installation mass addition. To account for additional miscellaneous items, a 5\% margin is added onto our estimate for the final gross weight. Component weight breakdowns and comparisons of results with existing aircraft are presented in the Validation section. 


\section{Performance}

Analysis of the flight performance of the aircraft is an integral part of flight planning, mission analysis, and constraint analysis. As a module in the design environment, the performance analysis is completed last. Structuring an N-squared diagram or Design Structure Matrix (DSM) involves placing processes at the end if they have no dependants [32], and the outputs of the performance analysis block are used only for constraints in the optimizer. As previously mentioned, the mission analysis and flight planning tasks involve some processes similar to those contained in the performance module, but the application of the tasks is slightly different here. The main goal for flight planning was to optimize flight $C_{L}$ at different stages for minimum energy or minimum power. In the performance analysis block, the focus is on finding maximum capabilities of the aircraft. Most important is an estimation of the absolute ceiling, maximum rate of climb, best sustained turn radius, dash speed, and takeoff distance.

The central process for calculating performance is an excess power function based

on the fact that $P_{S}=\frac{(T-D) V}{W}$. Since we are dealing with propeller aircraft, this expression should use power available rather than thrust available. Also, the routines are written such that the weight of the aircraft is not necessary, using parameters like wing loading and power to weight ratio. Lastly, it is desirable to express power in terms of Watts, so a conversion factor is necessary. $\kappa_{W a t t}=0.73756$ converts power from foot-pounds per second into Watts. The resulting expression for excess power is given in equation 7 .

$$
P_{S}=\frac{P_{W a t t}}{W} \kappa_{W a t t} \eta_{P}-\frac{C_{D} \rho V^{3}}{2 \frac{W}{S}}
$$


Excess power from equation 5 is synonymous to the rate of climb of the aircraft at the given speed and altitude, and we may approach the analysis from two perspectives. If the aircraft size is not known, $C_{D}$ must be based on an assumed drag polar. This allows us to evaluate point performance qualities of a scalable aircraft in terms of wing loading, power to weight, aspect ratio, etc. Alternatively, if the size of the aircraft is determined, reference and wetted areas are known and an accurate drag polar may be used to determine the actual performance metrics.

Many times, point performance requirements, expressed as constraints, leave a certain level of freedom open to the aircraft. For example, we may specify that the aircraft must exhibit at least 300 feet per minute rate of climb at a certain altitude, but not specify the speed at which it must occur. Reference [49] discusses some other requirements including a dash speed necessary to overcome the worst case $99^{\text {th }}$ percentile winds aloft, a statistical value for wind speeds that are only exceeded $1 \%$ of the time. A dash requirement may then be specified at either the cruise altitude or left free to be satisfied at any altitude. When there is such a degree of freedom, the performance calculations are subject to a minimization routine where the free variable like velocity or altitude is determined for best performance. MATLAB's fminbnd is generally what is used in this case where single variable functions are minimized on a fixed interval. Note that we usually want to maximize the quantity, so fminbnd applies a negative sign to the function in question. Figure 37 shows the core of the performance analysis process employed herein. 


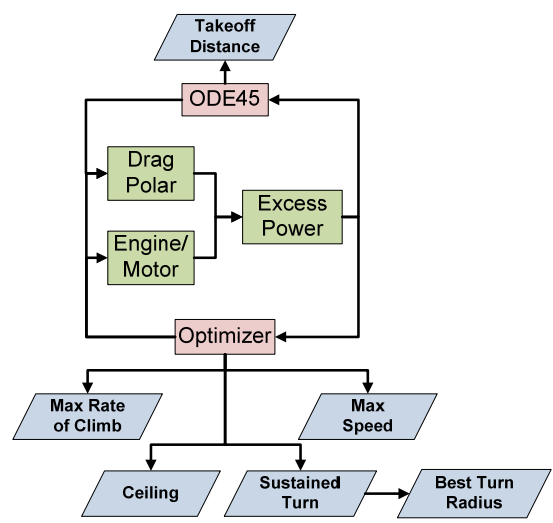

Figure 37 - Performance Analysis Process

Several performance charts are reproduced here in an effort to familiarize the reader with low wing loading values and power expressed in Watts. These performance calculations were completed with a simplified analytical drag polar and intend to demonstrate the operation of the process in Figure 37. The first point performance metric is the absolute ceiling, and constitutes one of the fundamental requirements or constraints on our system. Figure 38 below confirms that the high altitudes of interest to a HALE UAV drive the designs to aspect ratios on the order of $1 \mathrm{lb} / \mathrm{ft}^{2}$.

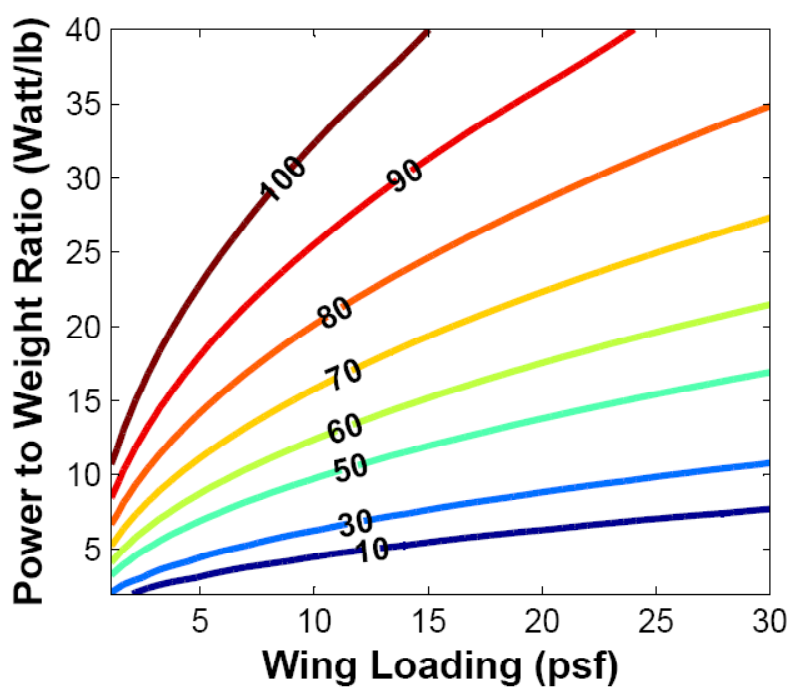

Figure 38 - Absolute Ceiling (kft) 
With the areas of wing loading and power-to-weight favorable to high altitude flight in mind, we see from Figure 39 that minimizing power that will allow a 100,000 ft ceiling results in roughly $400 \mathrm{ft} / \mathrm{min}$ maximum rate of climb at sea level. Also, for the design space shown, maximum rate of climb is much more dependent on power-toweight than on wing loading.

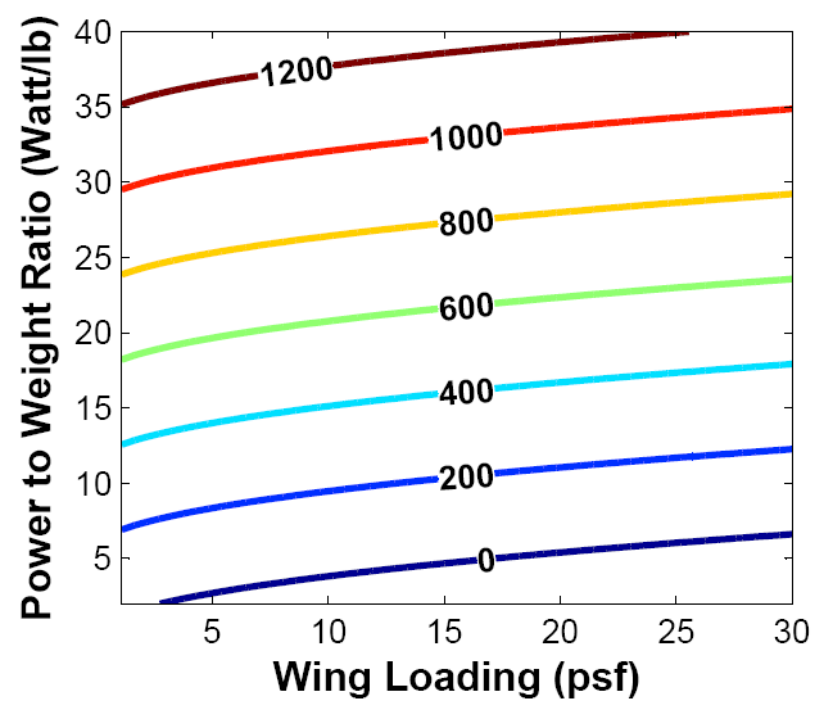

Figure 39 - Max Rate of Climb (ft/min)

When operating in the integrated MDO environment, this performance code will have more information available, like aircraft weight, detailed drag polars, and one specific design point wing loading and power-to-weight. These extra details take the place of the assumptions made to create the plots seen above, and rather than plotting over the whole design space, required performance metrics are calculated for just one point. 


\section{The Integrated Environment}

Assembling the individual disciplines and analysis/design modules discussed above finally provides the multidisciplinary analysis and design environment that we desire. Figure 40 shows a generalized schematic of how the disciplines communicate, and how the parameters of this environment may be controlled with an optimization routine.

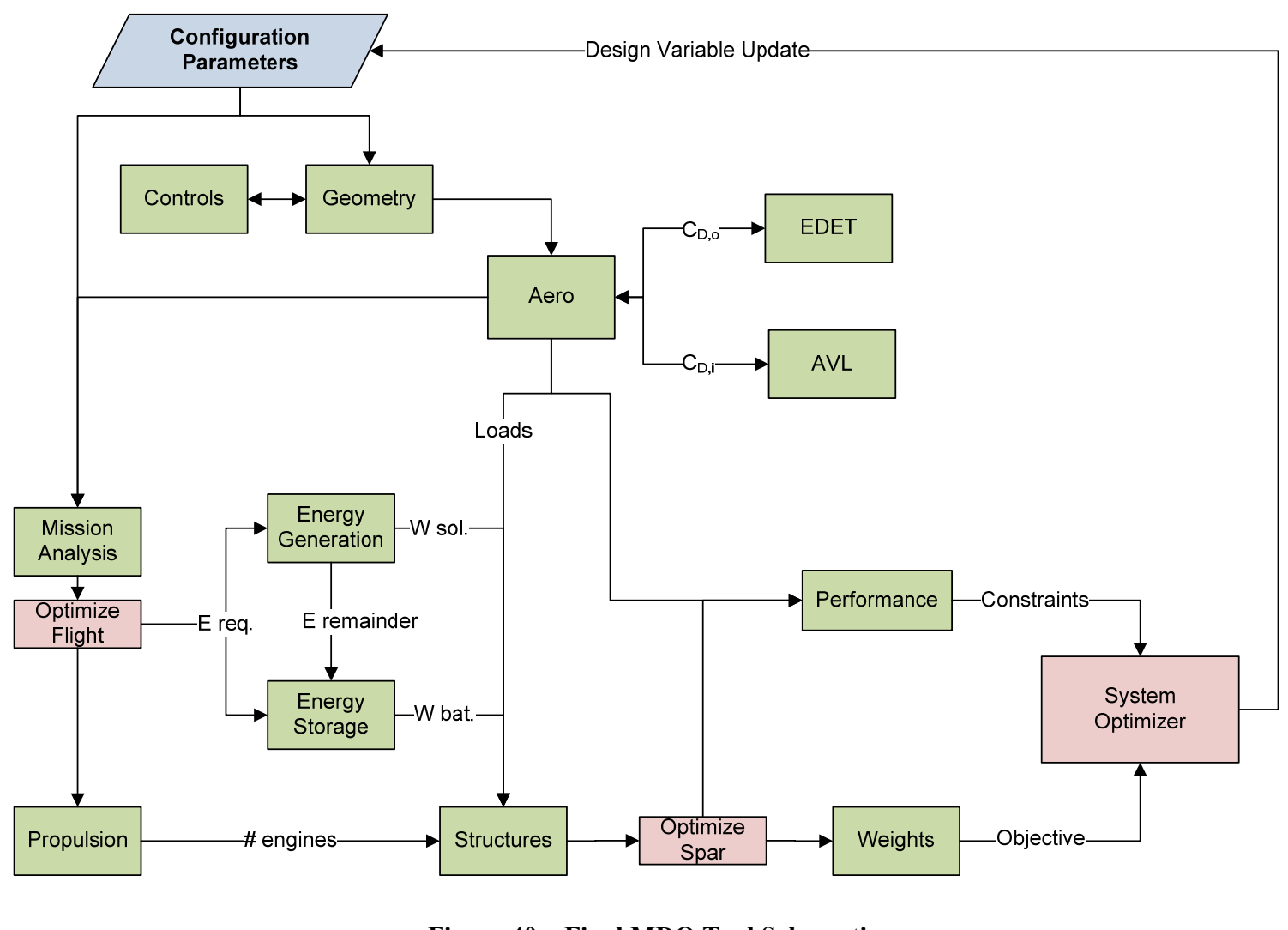

Figure 40 - Final MDO Tool Schematic 


\section{TOOL VALIDATION}

For a design tool to be at all useful, it must be checked to ensure some level of accuracy. Here, an attempt is made to explore the basic behavior of the MDA system and perform initial tests for sensitivities, and optimizer integration. The basic configuration modeled for the validation cases is a flying wing aircraft utilizing solar energy generation and advanced battery energy storage. This configuration allows comparison of results with the Helios Prototype aircraft. Several variants of Helios were flown, ranging from initial checkout flights powered entirely with batteries to the HP01 aircraft that reached record altitude to finally the ill-fated HP03 fuel cell powered long endurance configuration.

It is important to note that the goal for this study is to provide a design environment to support investigation of incremental benefits and sensitivities of various HALE UAV concepts. These parametric studies rely on tools that correctly model certain physical responses to changing inputs, but the focus is centered more on capturing the delta than finding exactly the quantity. Consequently, the purpose of a validation study is to ensure that the results are within a reasonable order of accuracy and calibrate areas of error. If the user determines that the MDA results are unacceptable, the task of identifying the suspect disciplines and replacing the module with one of greater accuracy is not a difficult one. Unfortunately, commonly available technical information for Helios is not highly detailed (Table 9), but should serve our purpose for this study. Building an approximation of Helios in our MDA environment involves specifying certain configuration inputs (Table 10) and evaluating the resulting aircraft. 
Table 9 - Helios Specifications [21]

\begin{tabular}{|l|l|}
\hline Helios Characteristic & Value \\
\hline Wingspan & $247 \mathrm{ft}$ \\
\hline Length & $12 \mathrm{ft}$ \\
\hline Chord & $8 \mathrm{ft}$ \\
\hline Wing Thickness & $12 \%$ \\
\hline Wing Area & $1976 \mathrm{ft}^{2}$ \\
\hline Aspect Ratio & 30.9 \\
\hline Empty Weight & $1,322 \mathrm{lb}$ \\
\hline Gross Weight & up to $2,048 \mathrm{lb}$ \\
\hline Payload & up to $726 \mathrm{lb}$ \\
\hline Solar Cell Efficiency & $19 \%$ \\
\hline Propulsion & $141.5 \mathrm{~kW}$ motors \\
\hline Endurance & daylight $+2-5 \mathrm{hrs}$ \\
\hline
\end{tabular}

Table 10 - Configuration Variables for Validation Case Input Variable Baselines

\begin{tabular}{|l|l|l|l|}
\hline Aspect Ratio & 31 & C $_{\text {Lmax }}$ & 1.5 \\
\hline Wing Loading & $1.0 \mathrm{lb} / \mathrm{ft}^{2}$ & \# Wing Pods & 5 \\
\hline Power to Weight Ratio & $11.0 \mathrm{~W} / \mathrm{lb}$ & Mission Profile & cruise $65 \mathrm{kft}$ \\
\hline Wing Thickness & $12 \%$ & Battery Technology & $132 \mathrm{~W}-\mathrm{hr} / \mathrm{kg}$ \\
\hline Spar Design & tubular C-fiber & Payload Information & $700 \mathrm{lb}, 2 \mathrm{~kW}$ \\
\hline Solar Properties & $20 \%$ eff. & Propulsive Efficiencies & Prop: 0.8 \\
\cline { 2 - 2 } & $90 \%$ coverage & & Power train: 0.69 net \\
\hline
\end{tabular}


Notice that several required input variables in Table 10 are not directly defined in the available information, specifically CLmax and the propulsive efficiencies. Also, the extra flight time and mission profile information may vary flight-to-flight with Helios. These characteristics are major design drivers, so for the first analysis case, some baseline values are assumed and a sensitivity analysis will be presented. Table 11 shows the analysis results for a baseline approximation of the Helios platform.

Table 11 - Baseline Validation Results

\begin{tabular}{|l|l|l|l|l|}
\hline Characteristic & Helios & MDA tool & Difference & \% Error \\
\hline Wingspan (ft) & 247 & 258.08 & -11.08 & $4.49 \%$ \\
\hline Chord (ft) & 8 & 8.33 & -0.33 & $4.06 \%$ \\
\hline Wing Area (ft ${ }^{2}$ ) & 1976 & 2148.55 & -172.55 & $8.73 \%$ \\
\hline \# Motors & 14 & 16 & -2 & $14.29 \%$ \\
\hline Gross Weight (lbs) & 2048 & 2148.43 & -100.43 & $4.90 \%$ \\
\hline
\end{tabular}

Error circa $5 \%$ on gross weight is encouraging, but it is prudent to investigate a bit farther. As mentioned earlier in the paper, the weight of the aircraft in the MDA environment is broken into payload, energy generation and storage, wing/tail structure, and propulsion components. Figure 41 shows the breakdown of gross weight by category for the baseline MDA, and highlights a slight discrepancy compared to our validation aircraft. The empty weight of Helios was quoted at 1,322 lbs, and the baseline analysis given here shows an empty weight of 800 pounds. Differences here are heavily dependent on the spanwise loading distribution, of which limited information is available for HP01, as well as on the battery scheme included. There are several methods of accounting for 
differences in cases like this such as implementing a correlation factor, possibly in the form of increasing the $5 \%$ miscellaneous item weight.

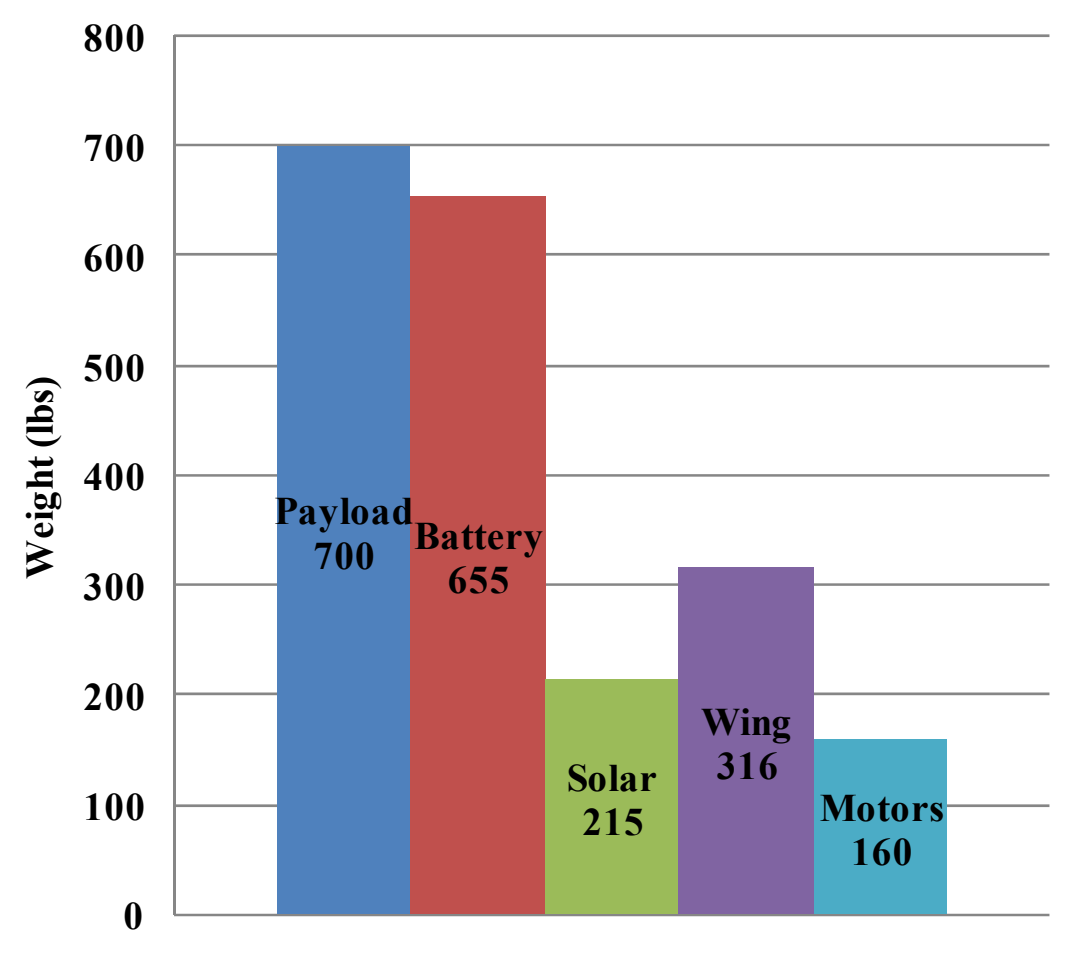

Figure 41 - Baseline Weight Breakdown

Before making adjustment to the tool, we must consider that some of the information that Figure 41 is based on was assumed. Also, recall that the focus here is primarily to evaluate the trends intrinsic to the design tool as long as it provides generally accurate results. Accordingly, an effort is made here to investigate the sensitivities to some of the key configuration inputs, specifically the ones where limited information is available and assumptions are necessary. For the following parametric study, a baseline configuration is defined by Table 10 and certain values are perturbed one at a time. 


\section{Maximum Lift Coefficient Response}

A nominal $C_{\text {Lmax }}$ of 1.5 was used to obtain the results of Table 11 and Figure 41. In general, the maximum lift coefficient of a HALE aircraft has a much greater effect on the mission performance than it does for conventional aircraft. The combination of a high aspect ratio wing and an extremely low wing loading decrease the induced drag of the aircraft drastically, causing the airspeed for minimum power to drop. In many cases, this minimum power flight condition may be below the stall speed of the planform. Figure 42 shows how this best power flight condition changes with increasing aspect ratio for a generic HALE type of aircraft [53].

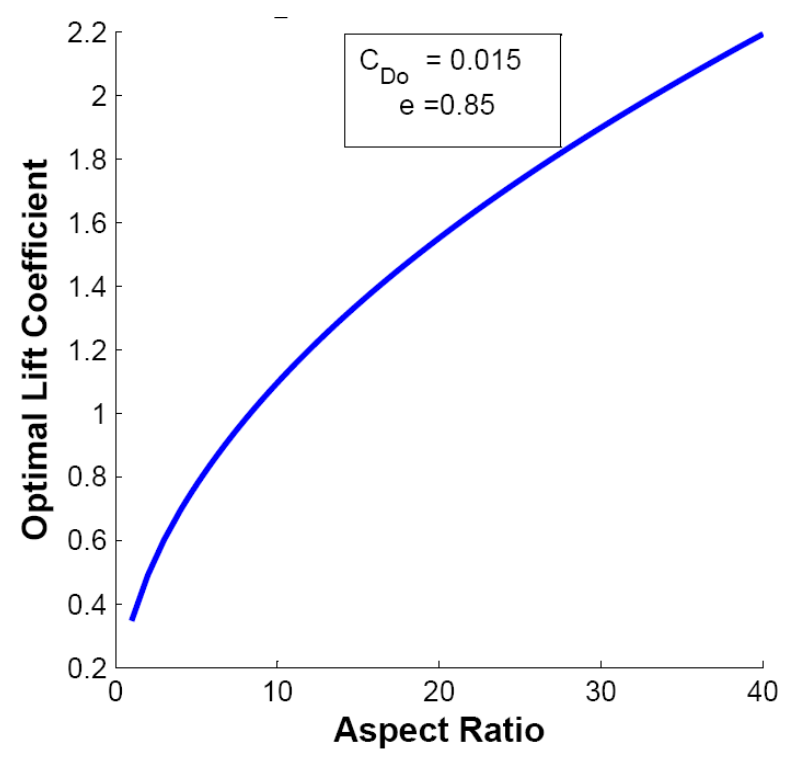

Figure 42 - $C_{L}$ for Minimum Power

It is clear that the flight planning and mission analysis of the vehicle desire a high lift coefficient, and this $\mathrm{C}_{\mathrm{Lmax}}$ should have a noticeable effect on the energy requirement and therefore weight of the resulting aircraft. Figure 43 shows the results of changes in maximum lift coefficient applied to a Helios - based design. 


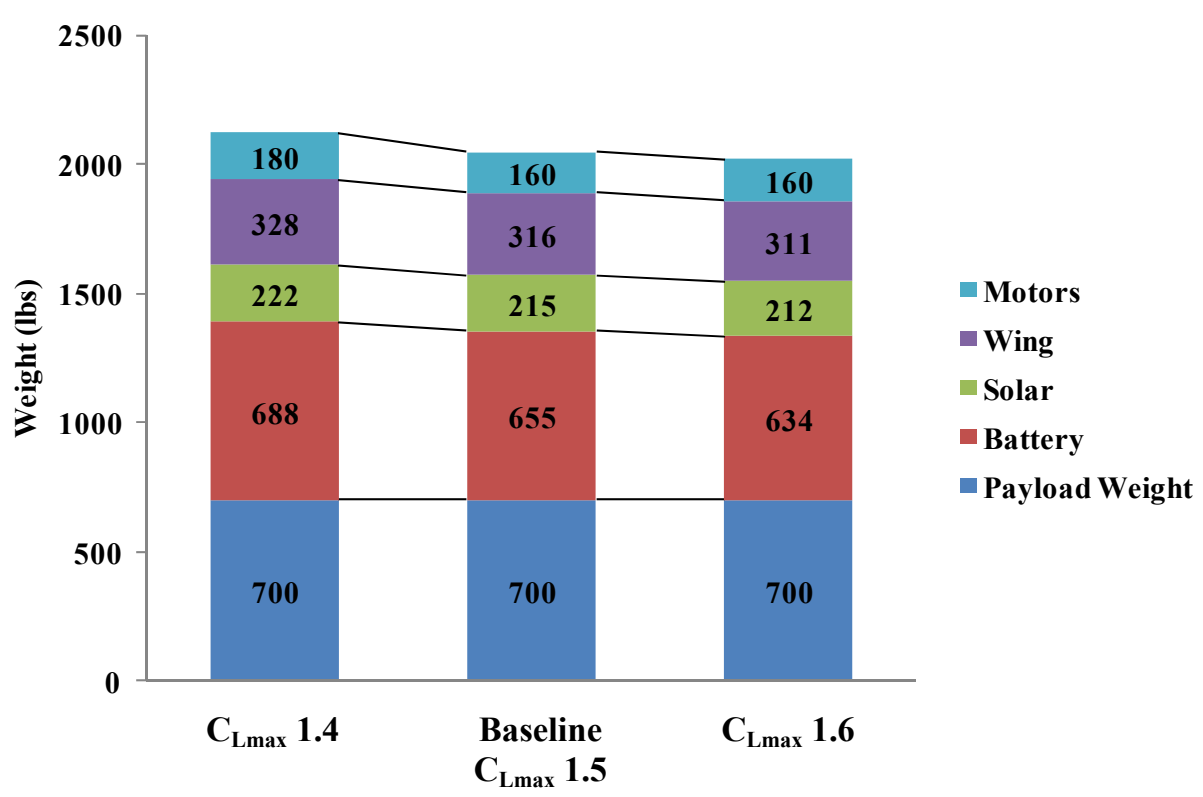

Figure 43 - Response to $C_{L m a x}$ Perturbation

When examining these results, one must keep in mind that the other pre-specified characteristics of the aircraft have not changed, most importantly the wing loading is a set value between the three cases of Figure 43. Also worth noting is that during flight planning and mission analysis, the lower bound for choosing a flight condition is 1.2 times $\mathrm{V}_{\text {stall, }}$ forcing the aircraft farther from the optimal point. What we see is that increasing the maximum lift coefficient does in fact allow the aircraft to operate at a more favorable $\mathrm{C}_{\mathrm{L}}$ as evidenced by the reduction in energy component weights. Also, the fact that the wing structural weight is sensitive to $C_{\mathrm{Lmax}}$ confirms that some of the finer interdisciplinary connections are working well. Specifically, the incremental increase in $\mathrm{C}_{\mathrm{Lmax}}$ has decreased the energy requirement, thereby decreasing the weight of the batteries for after-hours flight. The savings in battery weight translates to a slightly smaller wing for a given wing loading, reducing the weight of the solar power system and altering the load distribution of the wing resulting in an overall decrease in gross weight. 
Moving the other direction with $\mathrm{C}_{\mathrm{Lmax}}$ the design environment again exhibits expected behavior. Lower maximum lift coefficient forces our operating conditions off optimum and increases the energy requirement and gross weight of the aircraft. Also, the added power requirement exceeded the maximum available from the motors on the baseline, so two were added to maintain symmetry. For this design study, a $6.7 \%$ increase in $\mathrm{C}_{\mathrm{Lmax}}$ results in $1.4 \%$ decrease in gross weight while decreasing the max lift coefficient the same amount increases the gross weight by $3.6 \%$.

\section{Propeller Efficiency}

DARPA's Broad Agency Announcement for the Vulture Program specifically calls out "extremely efficient propulsion systems" as a potential area for funded development [5]. Helios included composite propellers designed to exhibit laminar flow at high altitudes for increased efficiency. In addition, the NASA Dryden Flight Research Center is developing a testing capability for subsonic small general aviation and UAV aircraft research, including topics like variable pitch props and real-time efficiency control loops [54]. From the standpoint of the design environment developed here, it is possible to integrate performance maps for a specific prop if desired, but if no information is available for the propeller, a constant prop efficiency $\eta_{P}$ is used. As with $\mathrm{C}_{\mathrm{Lmax}}$ information, detailed data concerning the Helios propellers is limited.

Propeller efficiency was briefly discussed in the propulsion section earlier, but it boils down to the fact that there is a certain mechanical power required for flight, and a higher $\eta_{\mathrm{p}}$ means less energy is wasted during conversions in Figure 31. Because this 
value is assumed during the validation analysis, again we want to evaluate how much a change in the input affects the output of the system.

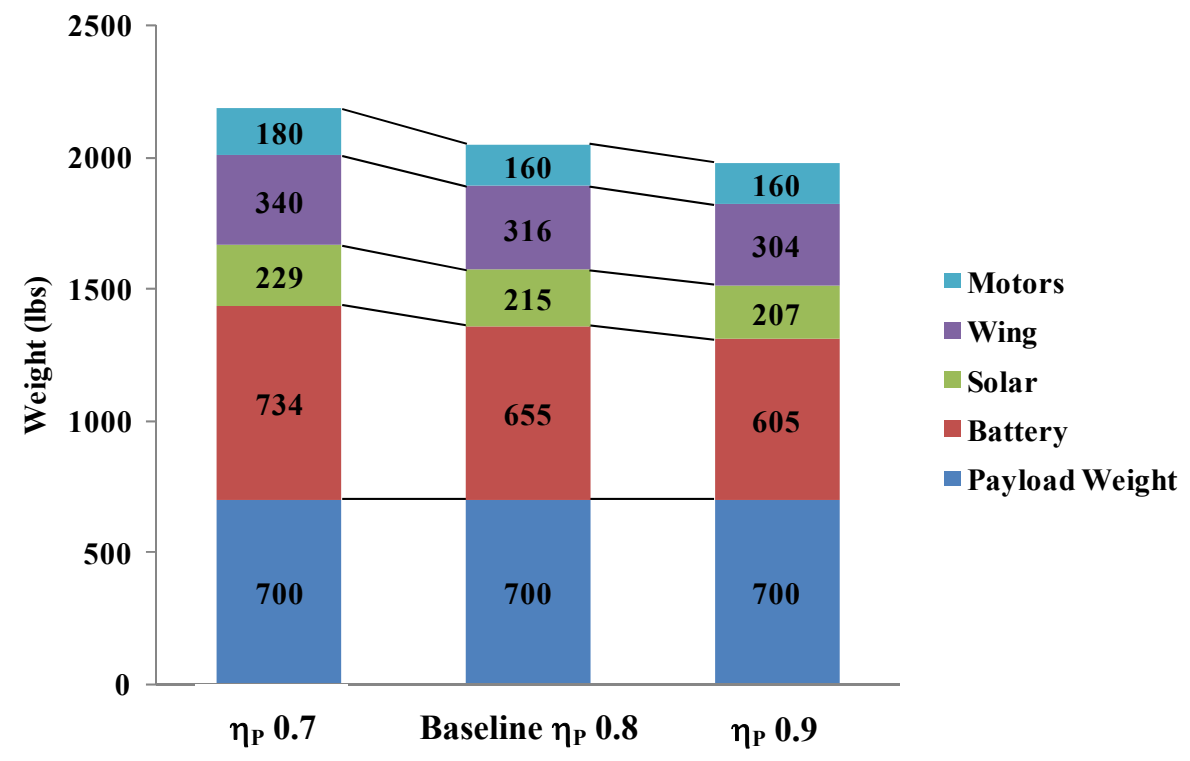

Figure 44 - Response to $\eta_{P}$ Perturbation

Again, the system responds as we expect, increasing efficiency drives energy requirements down resulting in overall weight reduction. Here, a $12.5 \%$ increase in efficiency gives $3.4 \%$ decrease in gross weight while decreasing the efficiency the same amount incurs a weight penalty of $6.7 \%$. Similar to the maximum lift coefficient sensitivity, given a certain change, the systems responds more to a detrimental delta than a beneficial one. 


\section{Target Altitude}

Ultimately, Helios was a prototype technology demonstrator with the eventual goal of demonstrating sustained flight to support any number of HALE UAV conops. A successful platform would ideally climb to its operational or target altitude, and either cruise or loiter for as long as possible. Though in practice and during the test flights the Helios aircraft may have deviated from this mission profile, the standard mission shown in Figure 11 is used as the design mission in the MDO tool. Specific data covering record setting flight profiles is not included and the difference in mission requirements may account for some discrepancies. Because there are many possible payloads and mission profiles to choose from, we must explore the performance of the aircraft for different target altitudes. Here, a target altitude is taken to mean the location of the main mission operation, and is separate from the ceiling of the aircraft, which may be much higher.

Conceptually, climbing to a higher operating altitude will require more energy, and should impose a weight penalty on the aircraft. Figure 45 confirms additional weight for higher altitude cruise conditions, but careful interpretation is necessary. Recall that Helios, weighing in at 2048 pounds or less was able to set flight altitude records in the mid-90 kft range. The difference is that the aircraft in the MDO environment is being sized to complete a day-long mission at that altitude and continue 2 hours after sunset at the cruise condition before beginning a descent. During the record setting altitude flights, Helios reached an altitude of 96,883 feet, but only sustained flight above the 96,000 foot mark for 40 minutes. 


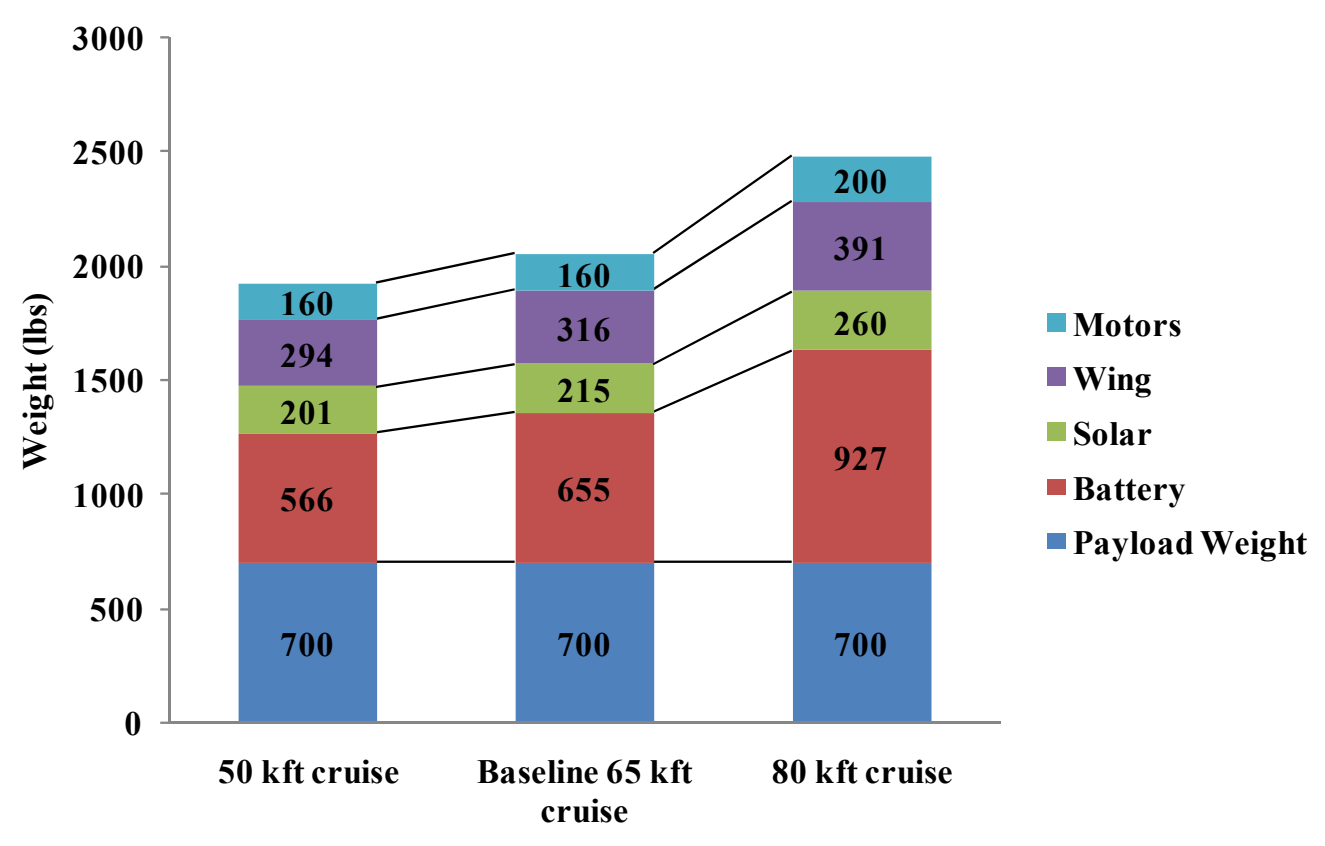

Figure 45 - Response to Target Altitude Perturbation

Once again, the penalty incurred for a change in input is greater than the benefit from the same change in a positive direction. Here, target altitude is altered $23.1 \%$ from its baseline value, and the aircraft grows $21.1 \%$ to perform the high altitude loiter compared to a $6.1 \%$ weight savings for operating at the lower altitude.

\section{Night Flight and Battery Requirement}

One of the largest determinants of the final weight of our HALE aircraft is the requirement for stored on-board energy. Most of the literature concerning the Helios Prototype mentions that it was capable of somewhere between one and five hours of operation after sundown [20], [21], [44]. The NASA spec sheet for Helios states that lithium batteries were used, but minimal information is available concerning mission 
specifics like at what flight condition the aircraft operated during the $2-5$ additional hours. There are several methods of accounting for an unknown factor like this after-dark flight capability in the design environment contained herein. First, the battery technology factor is scaled back to where state of the art batteries were in the late 1990's. In terms of specific energy density, advanced lithium-ion batteries may have achieved somewhere on the order of 130 Watt-hours per kilogram (roughly 60 Watt-hours per pound). Second, if the actual weight of the battery system implemented in Helios is known, it may be supplied to the design environment, producing the after-sunset flight capability an output. Alternatively, we may set the minimum required extra flight time as an input and the energy analysis modules cooperate with mission analysis to find the resulting battery weight necessary to continue the mission that long. Once again, as implemented, the extra time is on-station cruise or loiter condition flight, and the aircraft may begin descent afterwards.

For the baseline validation case, an additional flight time requirement of two hours was imposed. Of course we must evaluate how sensitive the resulting design is to this requirement. Figure 46 shows the aircraft gross weight sensitivity to changing night flight times. Here we see how drastically the additional endurance requirement affects the system. In addition, updating the battery energy density from the Lithium-ion technology to the current state of the art Lithium-Sulfur (Li-S) batteries increases the amount of energy storage per unit weight threefold. Figure 47 shows the resulting weight benefits. The baseline Lithium-ion aircraft with the ability to cruise 2 hours after sundown can save nearly $30 \%$ of its gross weight if upgraded to Lithium Sulfur technology. 


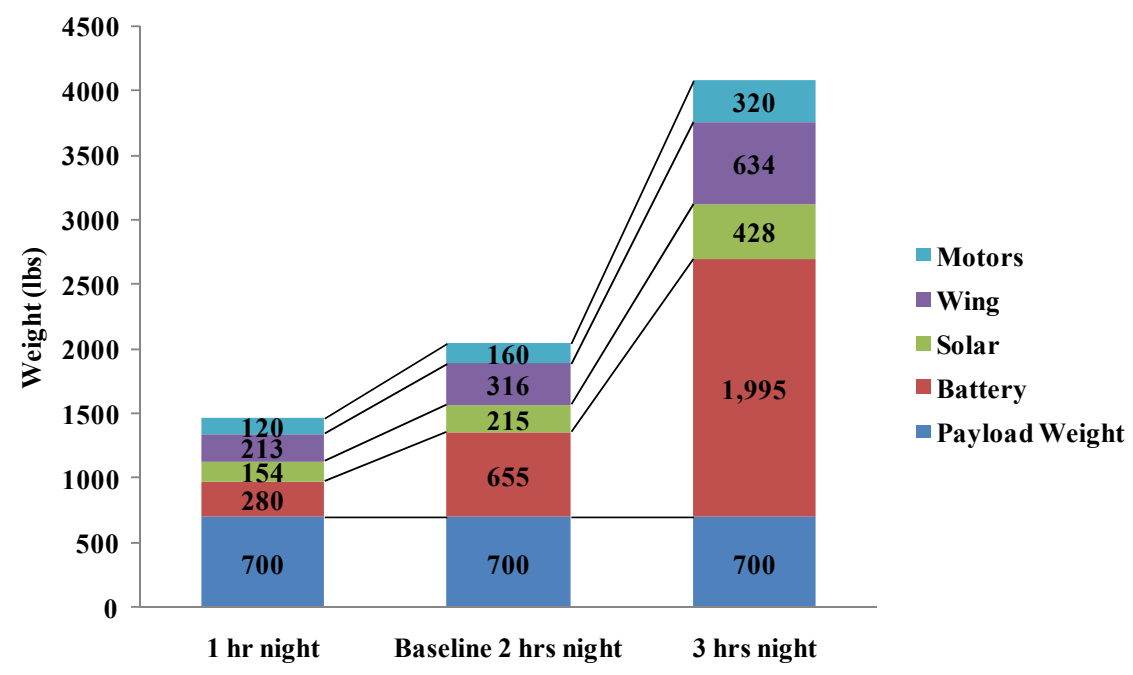

Figure 46 - Response to Night Flight Requirement

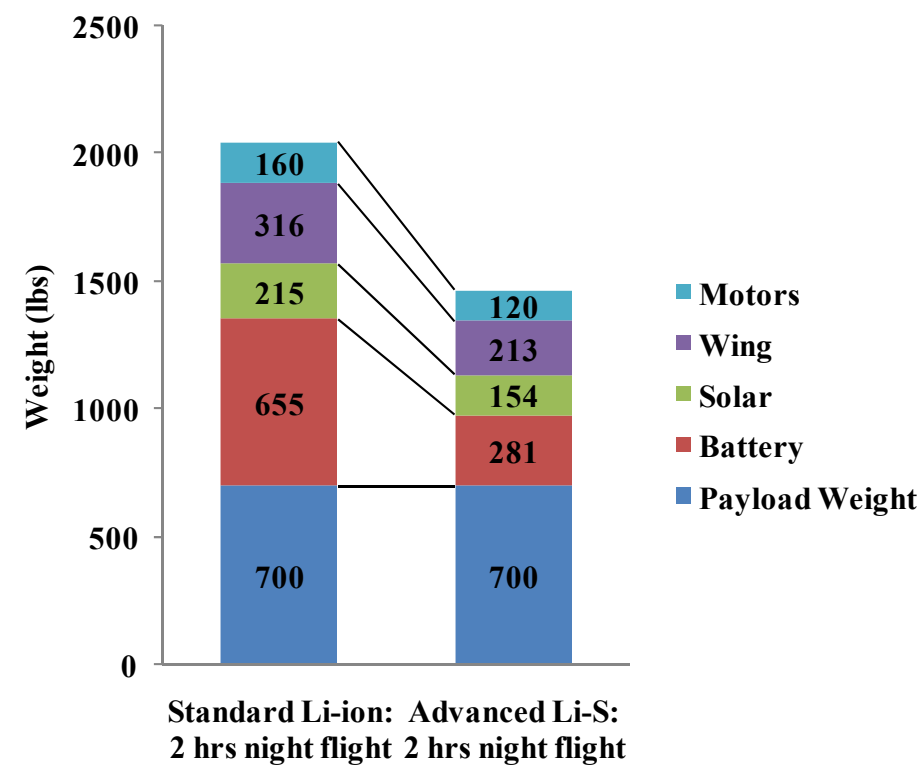

Figure 47 - Response to Improved Battery Technology 


\section{OPTIMIZING BASELINE ARCHITECTURE}

Evaluating benefits of the pinned wing concept can only be completed if there is a baseline capability to compare against. The first step is developing this basis for comparison is to build on the Helios validation case and introduce some extra degrees of freedom into the design. Progressively adding more design variables to an optimization routine will eventually build up the optimal baseline conventional HALE configurations that we need. First, the implementation of the Helios-style baseline aircraft described previously will be allowed to choose its own wing loading and power to weight ratio. The objective of the optimizer is to minimize the gross weight of the aircraft, and at first the only constraints specified are the convergence of weight, absolute ceiling of $100 \mathrm{kft}$, and a wingspan less than $300 \mathrm{ft}$. After solutions are found with only the two design variables, the same optimization is completed with the addition of aspect ratio as a free variable. At this point we may either continue to add more design variables to the optimizer for the validation mission concept of limited after-dark flight, or we may add the constraint for repeatable day/night energy cycles. Results for both avenues will be presented. Lastly, with the 24 hour constraint in place, the remaining design variables are unleashed and a full optimal solution to the two conventional mission concepts is sought. Please note that describing an aircraft as conventional here means that it is a one piece cantilevered or span loaded wing, contrasting the unconventional implementation of pinned wings. This process of building baseline optimal configurations is illustrated in Figure 48. 


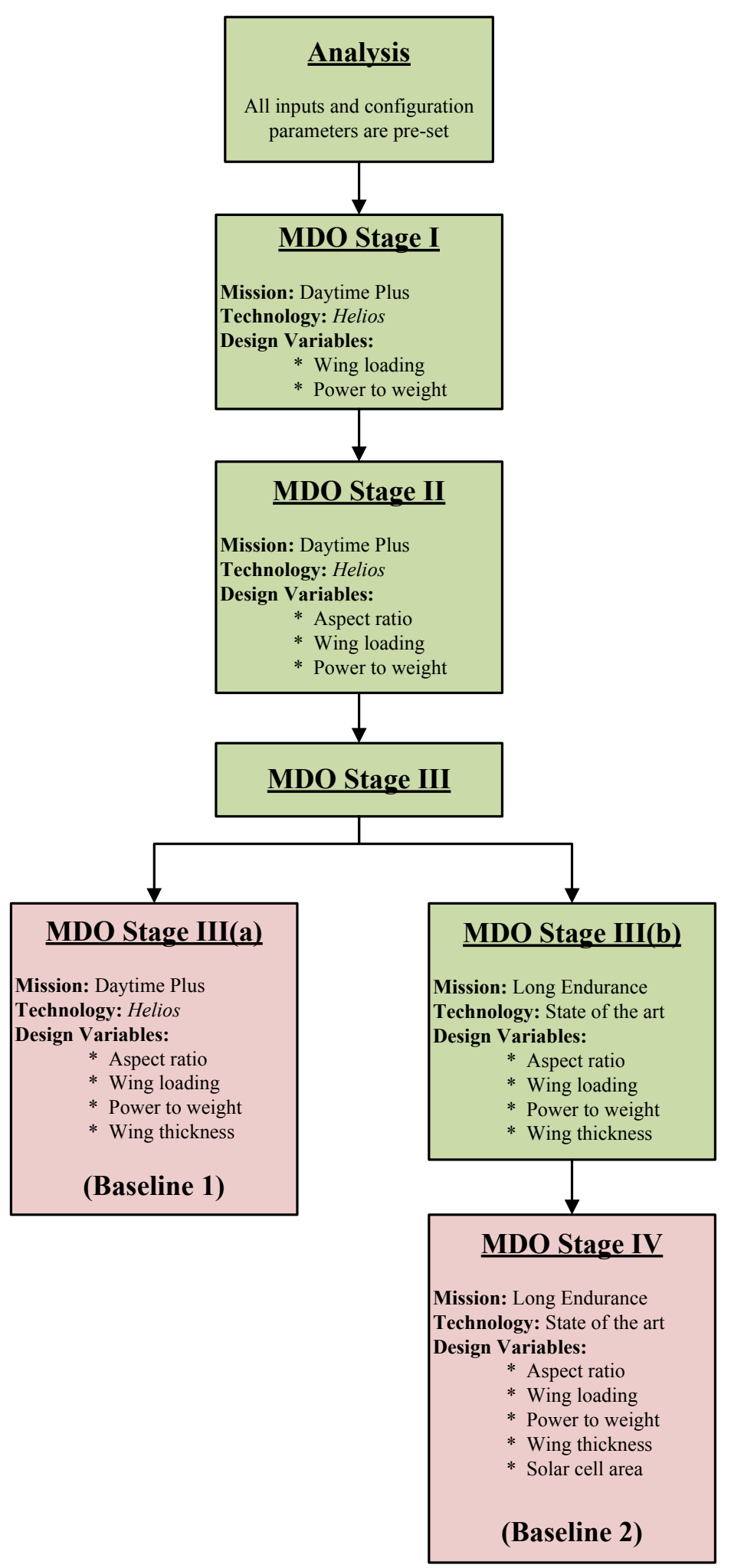

Figure 48 - Optimal Baseline Development Procedure 


\section{Stage I: Power and Wing Loading as Design Variables}

For the given Helios aspect ratio, wing thickness, technology level, and other characteristics of the baseline in Table 10, we now explore a design space of wing loading and power levels. Performing MDO on a complex system that uses a variety of analysis codes and table lookups tends to result in an unfavorable response surface for the objective function. We may expect local minima, or difficulty finding a feasible point. Indeed, choosing a feasible starting point is nearly impossible because of the Partial Optimizer Based Decomposition of the feedback links. Since the convergence of the design is a constraint imposed on the system, supplying a feasible point would mean that we would have already solved the system. In addition to the issue of local minima, the constraints on weight convergence pose another problem for finding the true optimum. Once the optimizer is able to make its way to a point where all the constraints are satisfied, where normally we would search for other feasible points that improve our objective, the optimizer for this effort has difficulty perturbing from one feasible point and finding another.

In order to explore as much of the design space as possible and find the overall best solution, the optimization is run many times for a variety of different starting points. The start point for each run is determined randomly based on a reasonable interval for each design variable. Table 12 shows the best results for the first stage of building an optimum conventional HALE UAV. 
Table 12 - Stage I Baseline Optimization Summary

\begin{tabular}{|l|l|l|l|l|}
\hline Design Variable & Start Point & Lower Bound & Upper Bound & Optimum \\
\hline Wing Loading (lb/ft $\left.{ }^{2}\right)$ & 0.815 & 0.5 & 5.0 & 0.875 \\
\hline Power to Weight (W/lb) & 14.13 & 0.6 & 60.0 & 7.44 \\
\hline
\end{tabular}

Progress of the design variables in this optimization run is tracked in Figure 49. As noted previously, the parameters of the optimization are scaled to be of the same order of magnitude, so the vertical axis in Figure 49 is scaled differently depending on which variable is considered. The scaling factors for this diagram are as follows:

- Gross Weight: scale up by 1000

- Wing Weight: scale up by 100

- Wing Loading: scale is 1 to 1

- Power to Weight Ratio: scale up by 10

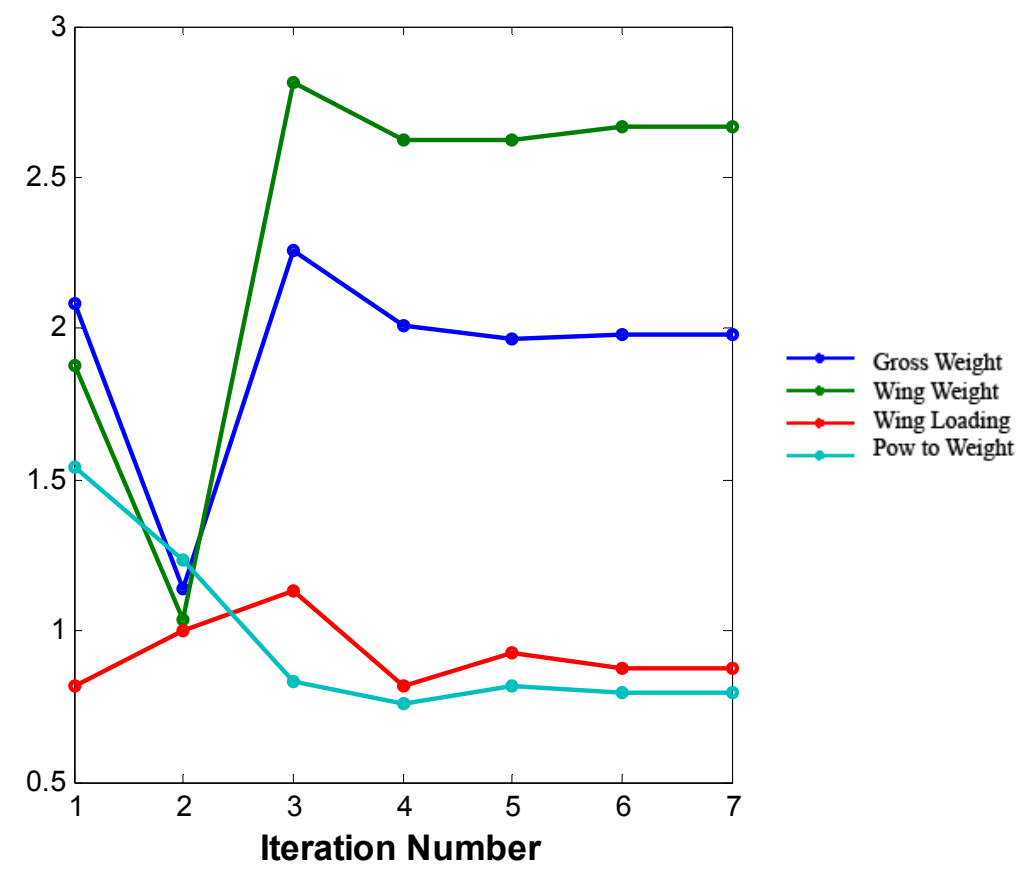

Figure 49 - Scaled Design Variable History 
Checking constraint violation helps to illustrate why some selections are made for design variables, Figure 50 shows the objective function history with points colored according to constraint violation. Green points designate that that the maximum constraint violation at that iteration was less than the specified tolerance.

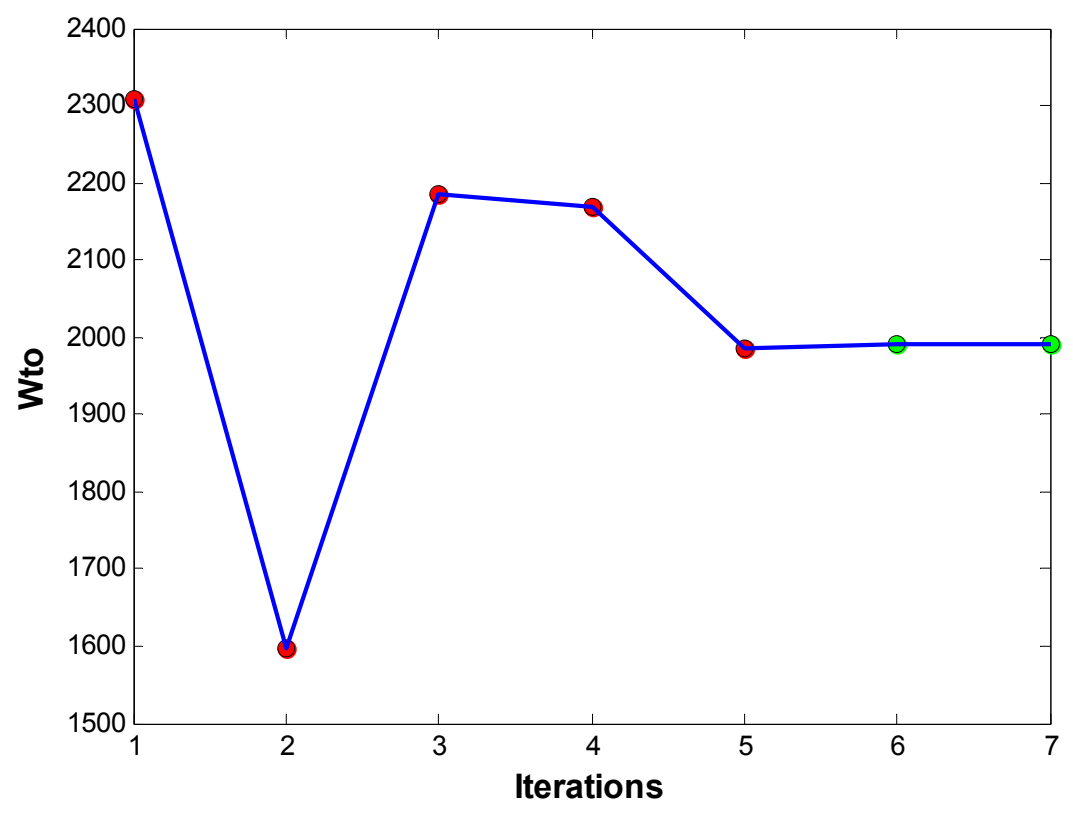

Figure 50 - Stage I Objective Function History

The results of this initial optimization case correlate well with the actual aircraft, as the wing loading for Helios varies by mission but is in the range of 0.8 to 1.1 pounds per square foot [21]. When compared with our baseline analysis (see Figure 51) the effect of decreased wing loading and power to weight is a decrease in weight because less energy is required for flight. 


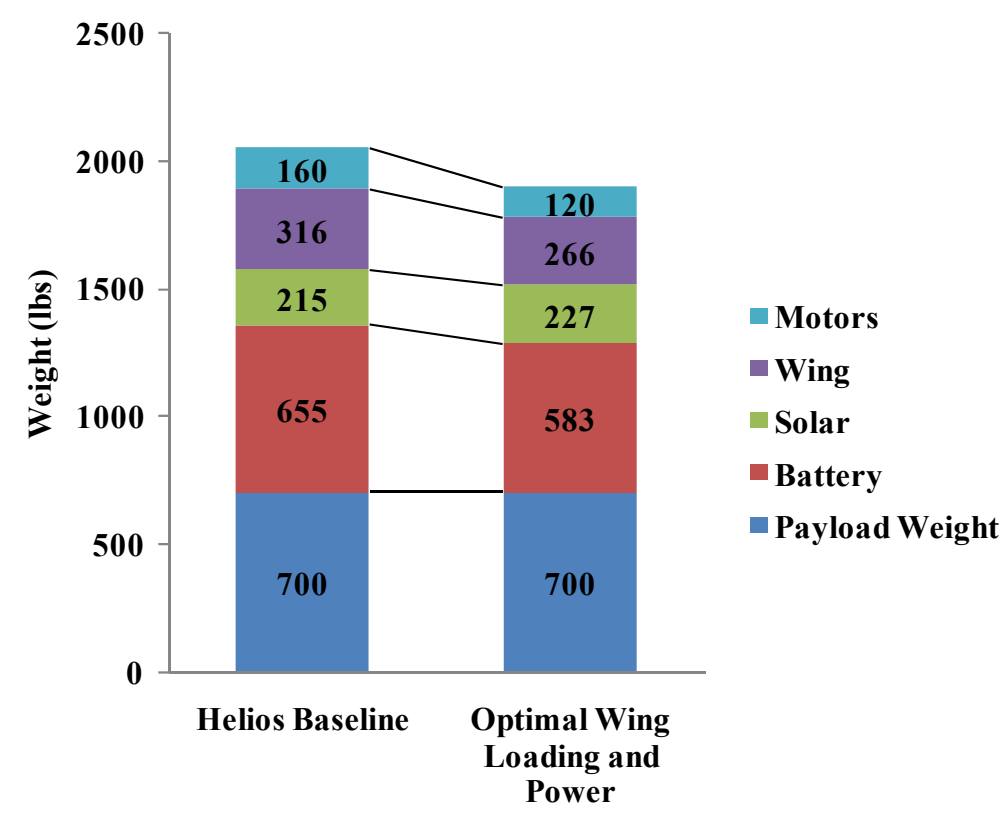

Figure 51 - Stage I Optimization Component Weights

\section{Stage II: Adding Aspect Ratio as a Design Variable}

The next step toward an optimal baseline configuration is the introduction of aspect ratio as a design variable. Here, again, constraints are imposed for absolute ceiling, wingspan, and design convergence, and the same method of using randomly generated start points is employed. Table 13 summarizes the design variable optimal values.

Table 13 - Stage II Optimization Summary

\begin{tabular}{|l|l|l|l|l|}
\hline Design Variable & Start Point & Lower Bound & Upper Bound & Optimum \\
\hline Aspect Ratio & 38.63 & 10 & 60 & 32.73 \\
\hline Wing Loading (lb/ft' $\left.{ }^{\mathbf{}}\right)$ & 1.04 & 0.5 & 5.0 & 0.854 \\
\hline Power to Weight (W/lb) & 16.45 & 0.6 & 60.0 & 7.55 \\
\hline
\end{tabular}

Design variable history is included in Figure 52 and shows that the additional

design variable has increased the number of iterations required. Also, the behavior 
exhibited here of making large initial steps and then slowly proceeding with minute changes is common for these optimization runs. As before, the variables are appropriately scaled in the MDO environment.

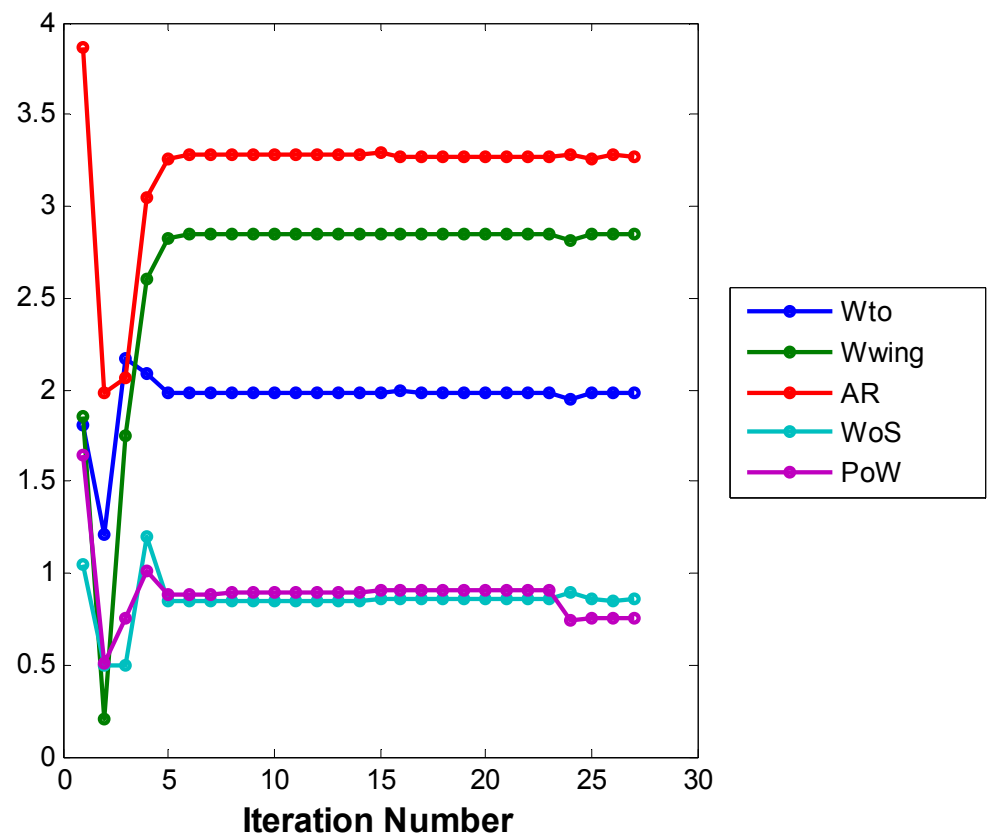

Figure 52 - Stage II Scaled Design Variable History

In this case, as the design variables make small perturbations to find new line search directions, Figure 53 shows that the constraints are not all met until the final iteration. Results of this optimization are that a slightly higher aspect ratio and lower wing loading may contribute a slight weight savings, $0.7 \%$ over the Stage I optimum but totaling $8 \%$ lower weight than the baseline (see Figure 54). Also, an interesting trend is shown in Figure 54 concerning wing weight. With a decreased battery and engine weight, the point loads along the span decrease, and the loss of their benefit for load alleviation necessitates a slightly heavier wing structure to keep a positive margin of safety. 


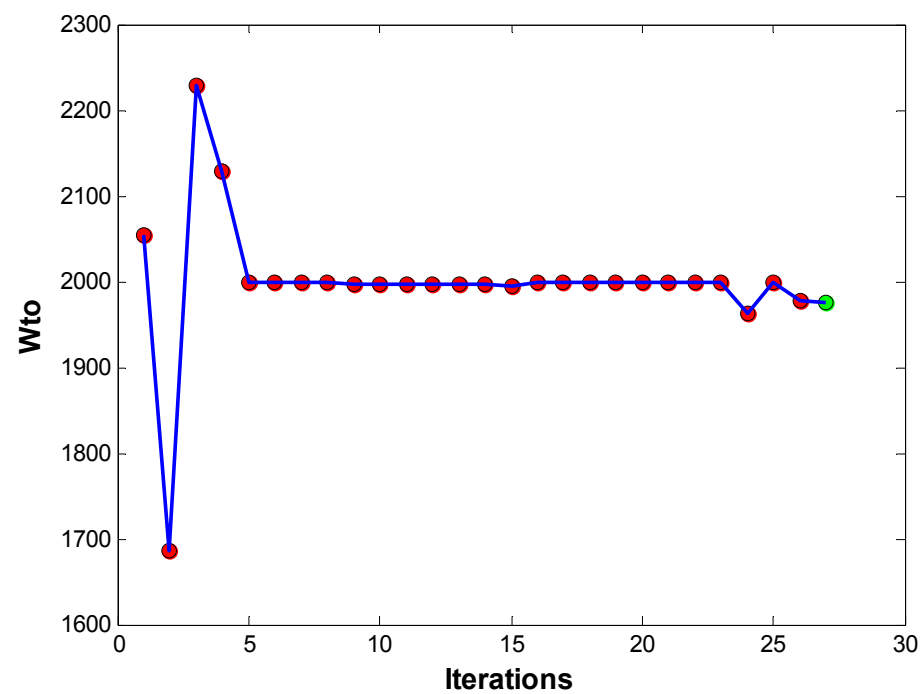

Figure 53 - Stage II Objective Function History

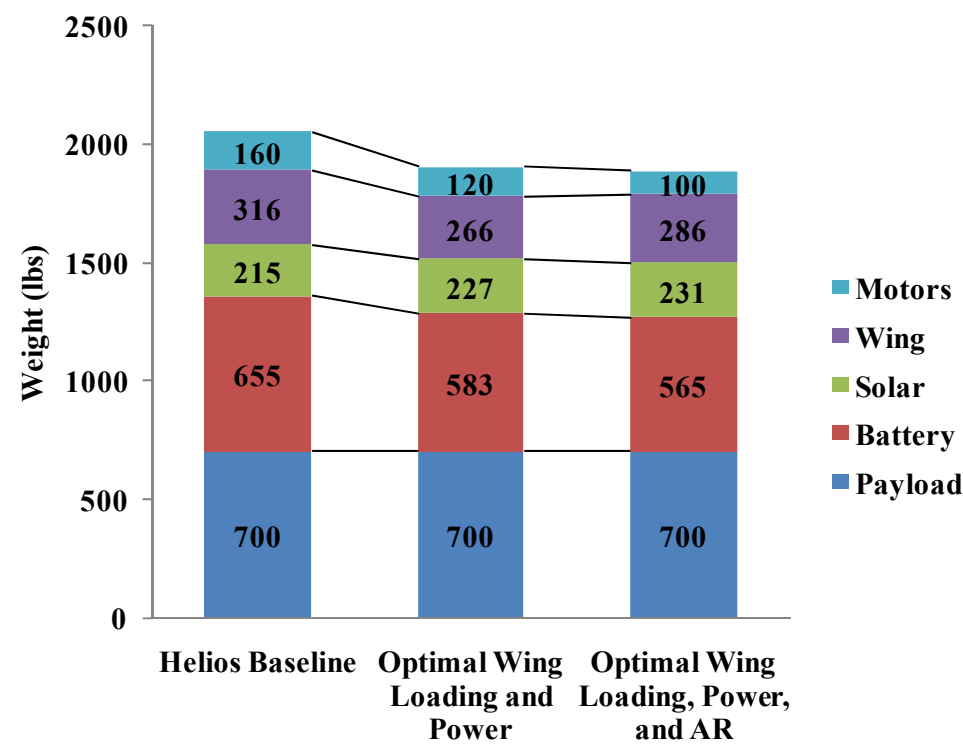

Figure 54 - Comparison of Optimal Weight Breakdowns

If we examine the actual constraint history (Figure 55) we see that the optimizer was close to a solution by the fifth iteration, and altering the tolerance on constraint violation may allow for decreased run times. 


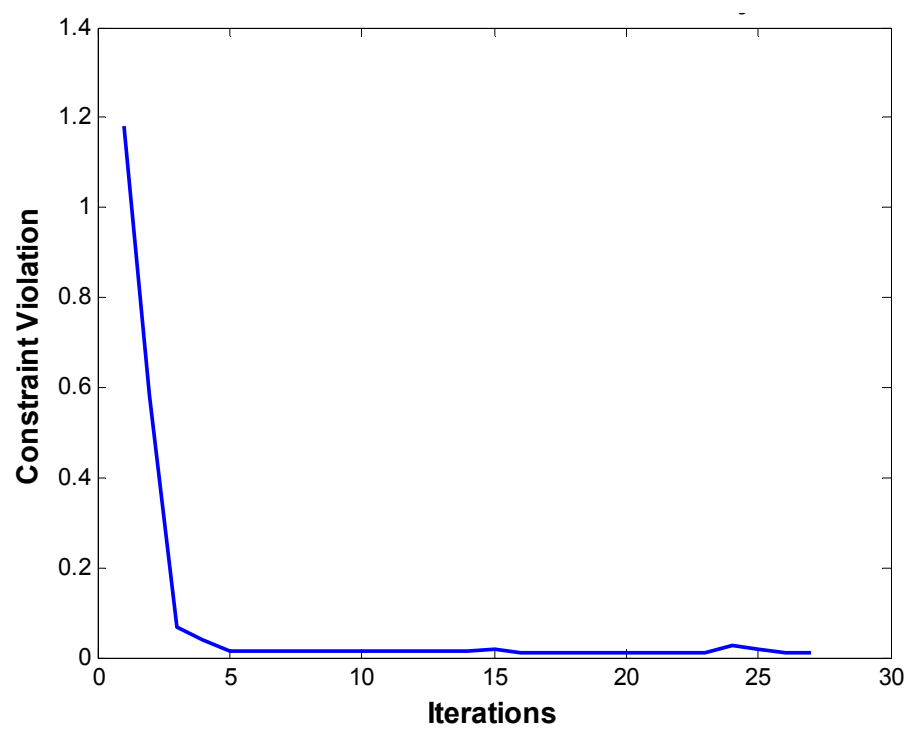

Figure 55 - Maximum Constraint Violation History

\section{Stage III a: Full Optimization of "Daytime Plus" Mission}

The final parameter to include in the optimization of the conventional aircraft platform covered here is the wing thickness. Up to this point, a $12 \%$ thick airfoil was included in the model because that is what the Helios Prototype employs. Changing the thickness of the airfoil should affect the mission analysis from a drag perspective, but the most drastic changes will most likely be seen in structural weight. Larger thickness allows for a larger spar cross section and thinner walls. Table 14 shows the design variable information, again with randomly generated start points. Wing thickness in this optimization has run into the upper bound, confirming that spar structural weight benefits from the increased thickness. Optimum values for the other variables have also changed, with the increased thickness allowing a slightly higher aspect ratio than previous runs. 
Table 14 - Stage III(a) Optimization Summary

\begin{tabular}{|l|l|l|l|l|}
\hline Design Variable & Start Point & Lower Bound & Upper Bound & Optimum \\
\hline Aspect Ratio & 29.84 & 10 & 60 & 37.62 \\
\hline Wing Loading (lb/ft ${ }^{2}$ ) & 1.16 & 0.5 & 5.0 & 0.826 \\
\hline Power to Weight (W/lb) & 11.96 & 0.6 & 60.0 & 7.28 \\
\hline Wing Thickness (\%) & 15.73 & 10.0 & 30.0 & 30.0 \\
\hline
\end{tabular}

Progression of the design variables toward the optimum is displayed below in Figure 56, again scaled appropriately so that the gold line representing thickness to chord ratio ends at a value of 3 but is implemented as $30 \%$ or 0.3 in the actual MDO environment.

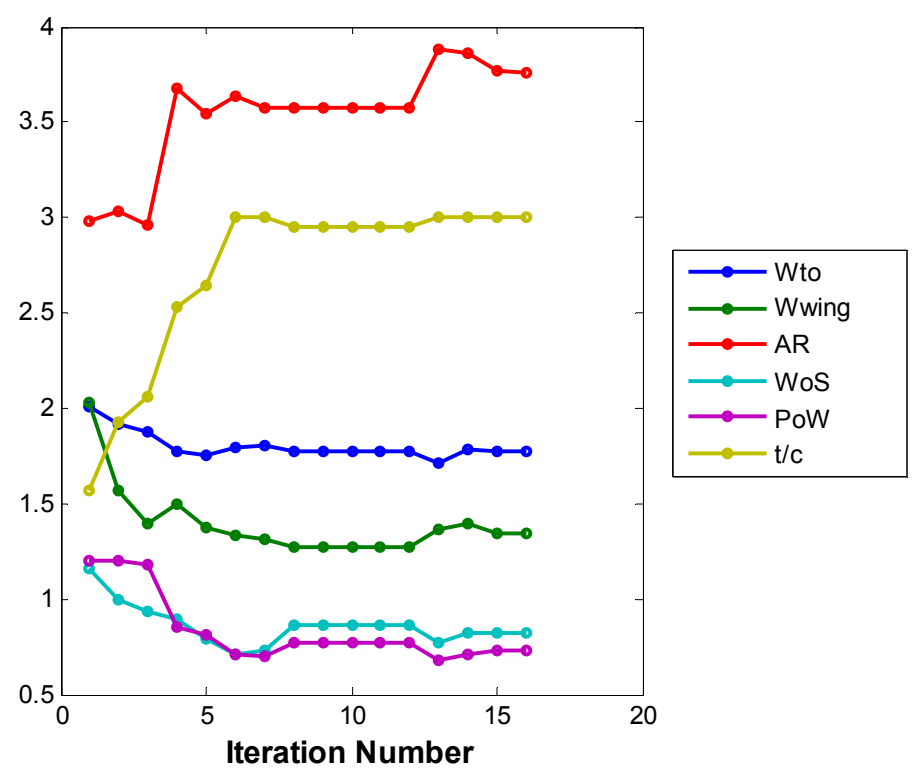

Figure 56 - Stage III(a) Design Variable History

As before, Figure 57 below charts the result of each line search, and the objective function value is tracked during optimization. Figure 57 shows that it is possible for the optimizer to perturb the first feasible point that it finds, and will sometimes violate constraints while searching for the optimum. 


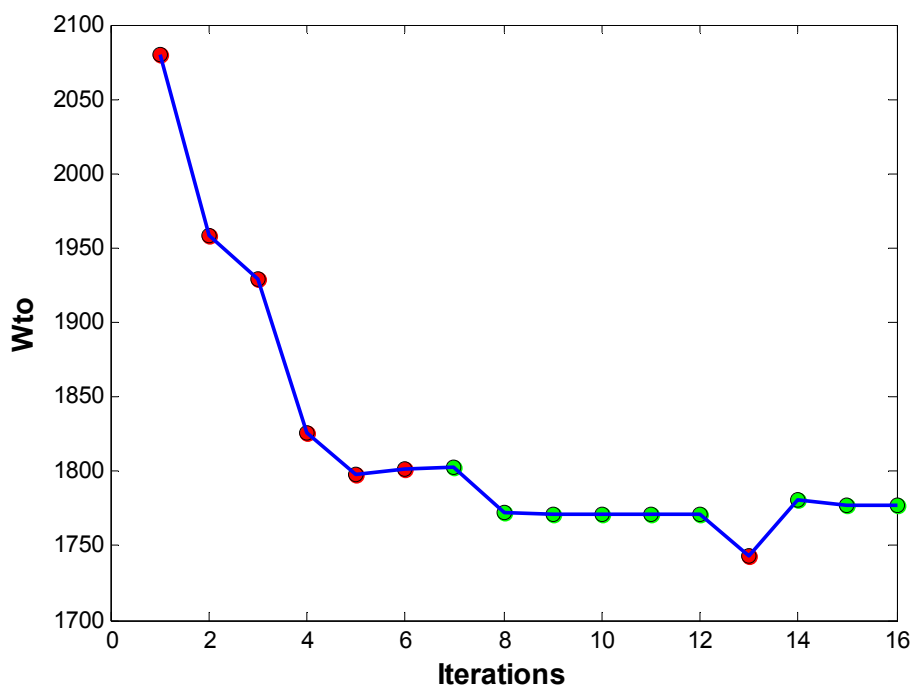

Figure 57 - Stage III(a) Objective Value History

The resulting weight breakdown for this full optimization of a limited endurance Helios proxy aircraft is shown compared to previous stage optima in Figure 58. The majority of mass categories for this case are quite similar to previous runs except when it comes to wing structural weight.

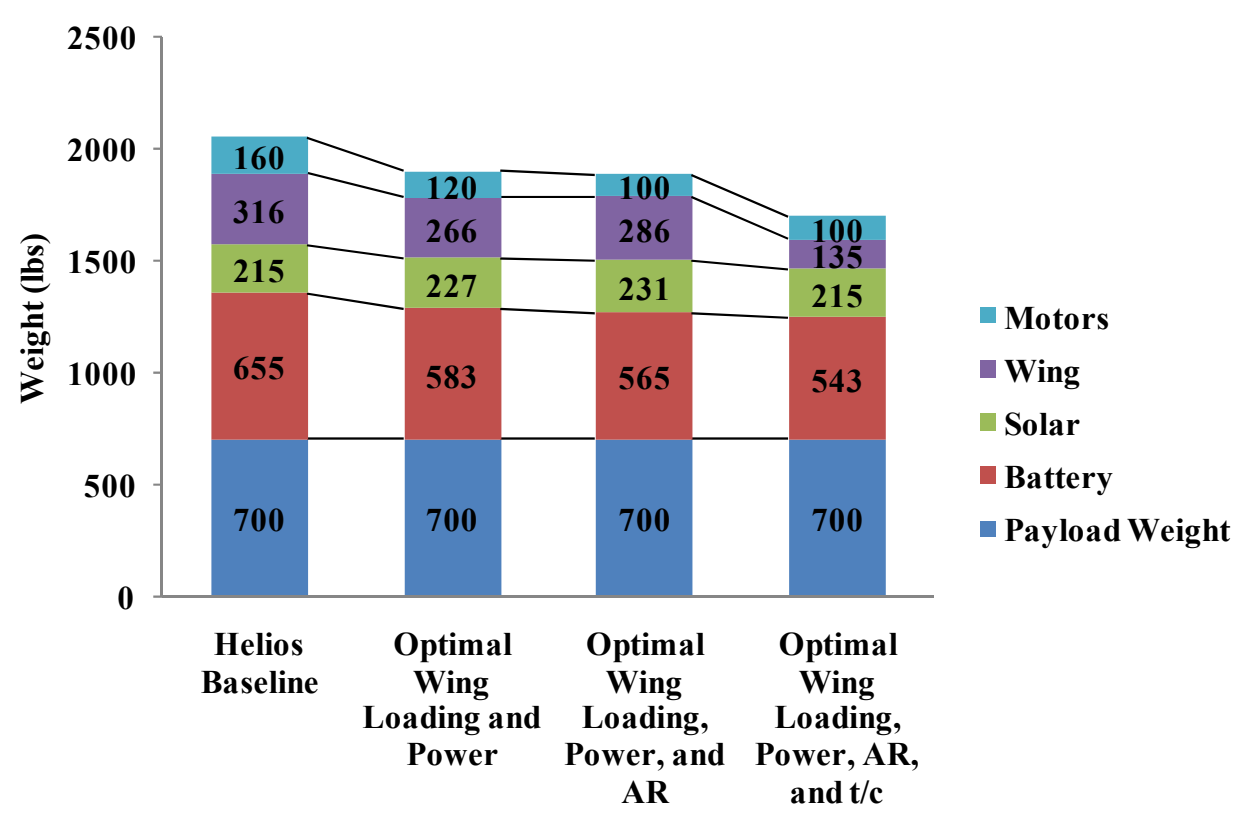

Figure 58 - Progression of Optimal Weight Breakdowns 
With a $30 \%$ thick airfoil, the spar weight may be reduced by $53 \%$ over the $12 \%$ thick airfoil of the previous case. In total, there is a $17.3 \%$ gross weight reduction from the initial baseline to the current optimal configuration.

Because the structural weight of the aircraft shows such a strong response to wing thickness, a few more details about each configuration are presented here. As previously noted, the spar is designed with a tubular cross section and is broken up into some number of sections. Maximum loading conditions are developed for each region of the spar and the wall thickness of each is individually minimized to meet a given factor of safety. For the MDO at hand, it is assumed that advanced composite construction techniques are used and that in reality the aircraft spar would exhibit a continuous thickness distribution. Accordingly, the spar of the baseline aircraft is divided into 10 sections for all MDO discussed in this paper. Figure 59 shows the discrete spanwise wall thickness profile for two aircraft configurations. Spar sections are numbered from aircraft centerline to wingtip. The blue bars in Figure 59 come from the baseline Helios proxy case, while the green is the most recent optimum configuration. Stage I and II optima fall in between the two cases shown.

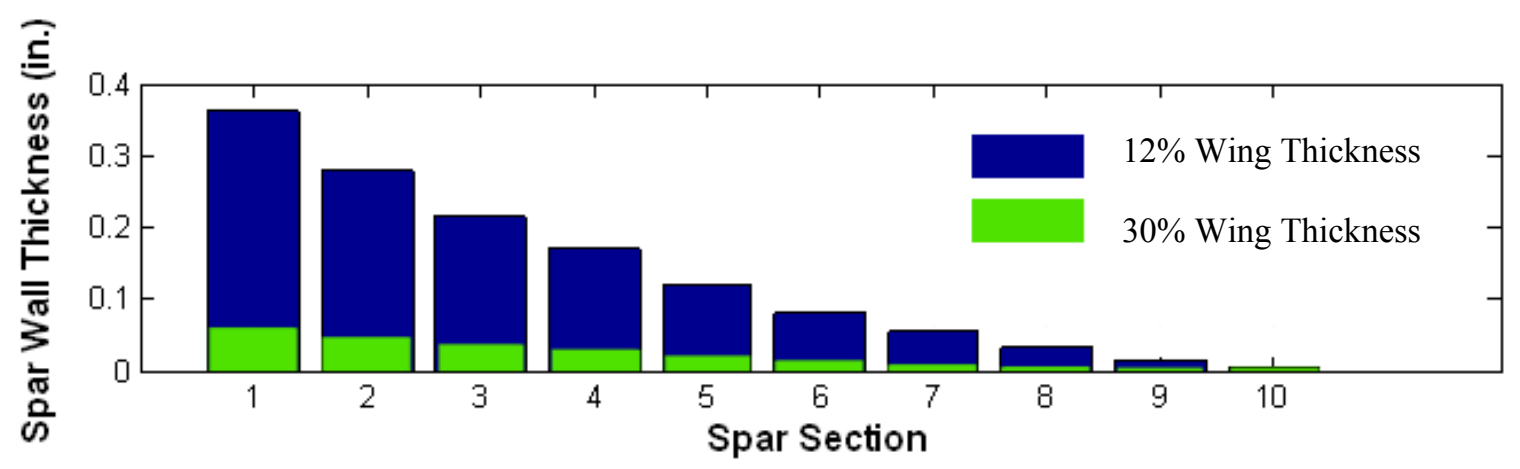

Figure 59 - Spar Design for Two Configurations 
Up to this point, we have focused on aircraft weight when comparing each successive optima. The progression has brought us to the stage where we have found an optimal baseline configuration from a design space with an acceptable degree of freedom, or number of design variables. Before moving on, however, there are other characteristics of each of these optima that have not yet been highlighted or compared to the original baseline design. A cross section of aircraft characteristics is included below (Table 15).

Table 15 - Optimization Data Summary

\begin{tabular}{|l|l|l|l|l|l|}
\hline & Helios & Baseline Proxy & Stage I & Stage II & Stage III(a) \\
\hline Gross Weight (lb) & 2048.00 & 2148.42 & 1990.13 & 1976.48 & 1776.90 \\
\hline Aspect Ratio & 31.00 & 31.00 & 31.00 & 32.73 & 37.62 \\
\hline Wing Loading (lb/ft ${ }^{2}$ ) & $0.8-1.1$ & 1.00 & 0.87 & 0.85 & 0.83 \\
\hline Power to Weight & 11.00 & 11.00 & 7.93 & 7.55 & 7.28 \\
\hline$S_{\text {ref }}$ & 1976.00 & 2148.51 & 2267.91 & 2315.56 & 2150.66 \\
\hline Wingspan & 247.00 & 258.08 & 265.15 & 275.31 & 284.43 \\
\hline Chord & 8.00 & 8.33 & 8.55 & 8.41 & 7.56 \\
\hline Wing Thickness & $12 \%$ & $12 \%$ & $12 \%$ & $12 \%$ & $30 \%$ \\
\hline Takeoff Distance & & 71.04 & 66.44 & 65.67 & 64.57 \\
\hline Max Climb Rate & & 359.82 & 253.52 & 241.73 & 234.41 \\
\hline \# Motors & 14 & 16 & 12 & 10 & 10 \\
\hline
\end{tabular}

The aircraft configuration of the rightmost column of Table 15 represents the Proven Technology Medium Endurance Baseline aircraft for this study. 


\section{Stage III b: Four Variable Optimization of Long Endurance Baseline}

The third stage of progressively adding design variables to the MDO is divided here to allow the exploration of a long endurance configuration. From the outset of this project the two inspiring platforms were the Helios Prototype and the Broad Agency Announcement for Vulture. If we are to design and optimize a pinned wing aircraft for sustainable day/night operation, we must develop an appropriate optimal baseline configuration as a standard. Stage III(a) delves as far into the battery powered Helios aircraft as we can, but never attempted to surpass the couple of hours of night flight that the aircraft demonstrated. Stage III(b) includes up to the wing thickness to the set of design variables, but also imposes the constraint for a sustainable regenerative energy balance. Also, several key technology factors are upgraded to current or projected nearterm values, including involving new power-system data given in [36]. Presented at the 2009 CAFE Foundation Electric Aircraft Symposium, the approach to sizing power- or energy-system components includes data concerning power train efficiencies and electric motor power densities. Other technology improvements are increased solar cell efficiency from $20 \%$ to $30 \%$ and upping the energy density of the batteries from 130 to 400 Watt hours per kilogram. The $400 \mathrm{~W}-\mathrm{hr} / \mathrm{kg}$ mark is based on Lithium-Sulfur batteries currently entering the market, but advanced nano-wire Lithium-polymer batteries may soon reach upwards of $700 \mathrm{~W}-\mathrm{hr} / \mathrm{kg}$ [36], [55].

Original technology and configuration definition parameters (Table 10) are updated for this Advanced Technology Long Endurance Baseline (ATLEB) and summarized below in Table 16. 
Table 16 - Parameters Defining the Long Endurance Baseline

\begin{tabular}{|l|l|l|l|}
\hline \multicolumn{4}{|c|}{ Advanced Technology Long Endurance Configuration Inputs } \\
\hline Mission Profile & cruise $65 \mathrm{kft}$ & C $_{\text {Lmax }}$ & 1.5 \\
\hline Battery Technology & $400 \mathrm{~W}-\mathrm{hr} / \mathrm{kg}$ & \# Wing Pods & 5 \\
\hline Spar Design & tubular C-fiber & Payload & $700 \mathrm{lb}, 2 \mathrm{~kW}$ \\
\hline Solar Properties & $30 \%$ eff. & $\begin{array}{l}\text { Propulsive } \\
\text { Efficiencies }\end{array}$ & Prop: 0.8 \\
\cline { 2 - 2 } & $90 \%$ coverage & & Power train: 0.92 net \\
\hline
\end{tabular}

Recall from Figure 46 that when determining the system sensitivity to battery powered flight requirements, an increase from 2 hours to 3 hours elicited an enormous response, effectively doubling the aircraft weight. That result discourages the idea of a solar-battery regenerative aircraft achieving continuous operation, but fortunately the design environment was approximating 15 year old technology. With the updates listed in Table 16, the optimization for a long endurance mission is completed and described below (Table 17). As before, the best solution drives to the upper bound of wing thickness, and the aspect ratio is in the high 30's.

Table 17 - Stage III(b) Optimization Summary

\begin{tabular}{|l|l|l|l|l|}
\hline Design Variable & Start Point & Lower Bound & Upper Bound & Optimum \\
\hline Aspect Ratio & 34.82 & 10 & 60 & 36.95 \\
\hline Wing Loading (lb/ft' $\left.{ }^{2}\right)$ & 0.86 & 0.5 & 5.0 & 0.77 \\
\hline Power to Weight (W/lb) & 13.89 & 1.0 & 60.0 & 7.07 \\
\hline Wing Thickness (\%) & 11.13 & 8.0 & 30.0 & 30.0 \\
\hline
\end{tabular}


Plots of design variable and objective function values are not outstanding for this case compared to others. Figure 60 compares the resulting aircraft weight breakdown to those previously developed. Notable results are that the increases in technology have resulted in an aircraft that carries only slightly more batteries than the original limitedendurance baseline, and that the total weight is just $5 \%$ higher than the optimized solution to the Helios proxy.

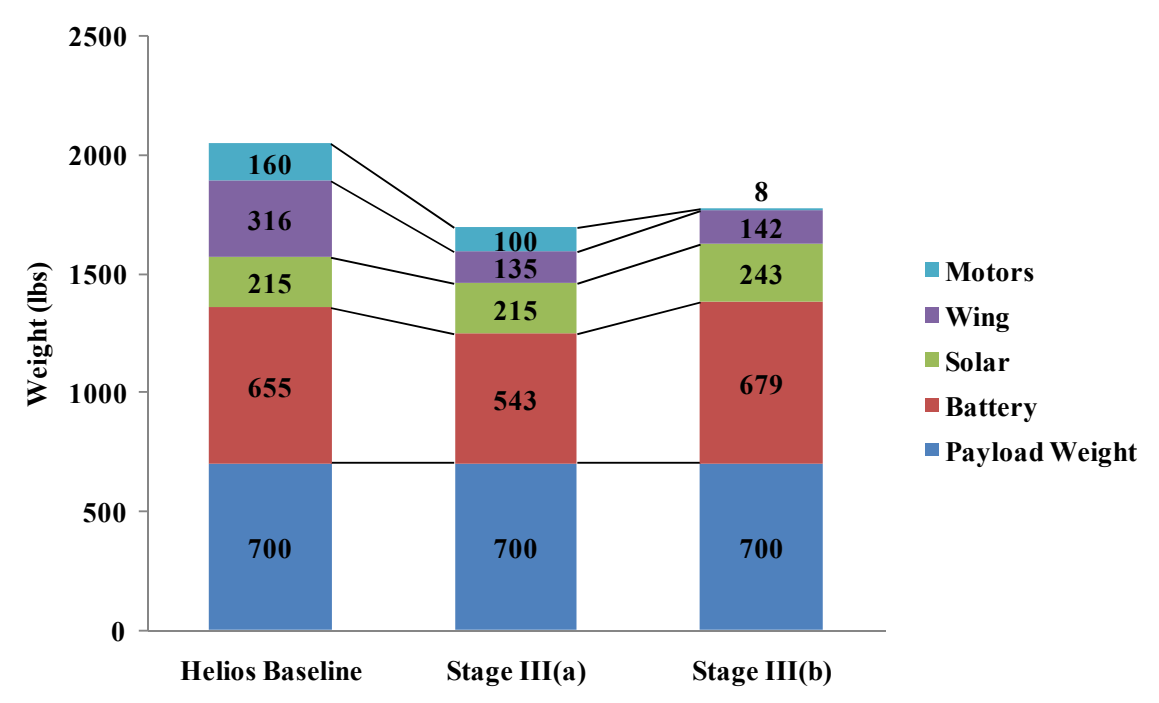

Figure 60 - Weight Breakdown for the ATLEB Compared to Earlier Results

One further note concerning the Stage III(b) optimization case is that the three inequality constraints mentioned in Table 5 are all active at the solution. The wingspan at this optimum is $300 \mathrm{ft}$, the absolute ceiling is $100,036 \mathrm{ft}$, and the batteries included store just enough energy to reach the time after sunrise when solar power is high enough to power the plane. Though earlier optimizations involved an active ceiling constraint, this is the first to push all the way to the upper bound of wingspan. The reason is that strenuous mission requirements here cause the airplane to desire low wing loading and high aspect ratio as energy storage penalties outweigh structural ones. 


\section{Stage IV: Full Optimization of Long Endurance Baseline}

The final step for our optimal conventional system is to allow the MDO tool to determine characteristics of the solar power system. Specifically, what we are looking for is a reduced solar coverage that will provide just enough power to fully charge the batteries while supporting flight and mission operations. In the previous stage of finding the optimal configuration, both the solar cell efficiency and the percent of wing covered were pre-set. The result of assuming too much wing coverage is shown in Figure 30. When the energy analysis modules are called in the design environment, they determine the total energy required to power flight, aircraft systems, and payload operation from the point when available solar power no longer exceeds the required amount to the corresponding time the following morning when roles are reversed. Now that the design environment knows the maximum charge needed, it simply caps the battery weight forming the plateaus in the battery charge timeline.

The goal of this final MDO phase is to decrease solar area so that the batteries are charged slowly, and reach full capacity just as the extra power from the solar array drops to zero. A summary of the results for this optimization can be found in Table 18 .

Table 18 - Stage IV Optimization Summary

\begin{tabular}{|l|l|l|l|l|}
\hline Design Variable & Start Point & Lower Bound & Upper Bound & Optimum \\
\hline Aspect Ratio & 34.50 & 10 & 60 & 22.66 \\
\hline Wing Loading (lb/ft' $\left.{ }^{2}\right)$ & 1.03 & 0.5 & 5.0 & 0.5 \\
\hline Power to Weight (W/lb) & 14.62 & 1.0 & 60.0 & 9.47 \\
\hline Wing Thickness (\%) & 13.85 & 8.0 & 30.0 & 30.0 \\
\hline Solar Area (\% S Sef) & 64.01 & 0.0 & 98.0 & 36.89 \\
\hline
\end{tabular}


Again, for this optimization, the wingspan constraint is active, but there are several aspects of these results that are suspect. First, the trend that we have observed up till now of increasing aspect ratio has reversed to a value lower than the initial baseline. Second, the wing loading design variable is at its lower bound, which in its own right is not necessarily alarming, but in the context that we have not seen that behavior before from the optimization cases it may be an area to investigate. In addition, we see in Figure 61 that during the third iteration the wing loading jumped to its lower bound and did not visibly move for the remainder of the operation.

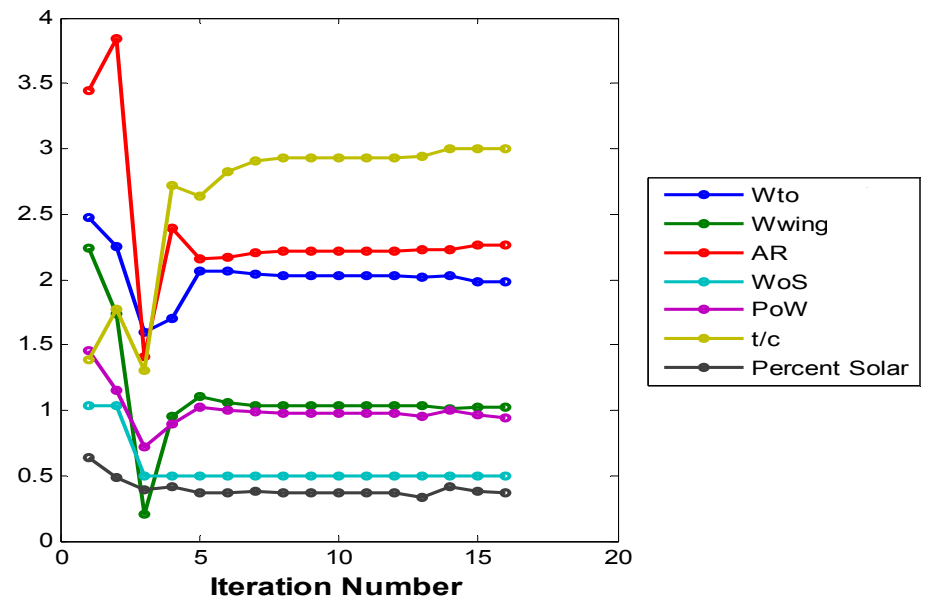

Figure 61 - Stage IV Design Variable History

Another area of question is that the power to weight ratio at the optimum has increased from what was previously a steady and predictable value. Because of the jump in power to weight, the ceiling of this configuration is $106,384 \mathrm{ft}$. It seems that the appropriate action of the optimizer would have been to decrease the power to weight ratio of the aircraft to reduce weight and remove the excess ceiling margin. 
The most incriminating result, however, is the actual objective function value, the aircraft gross weight. Figure 62 shows that the required battery weight has drastically increased over the previous stage result.

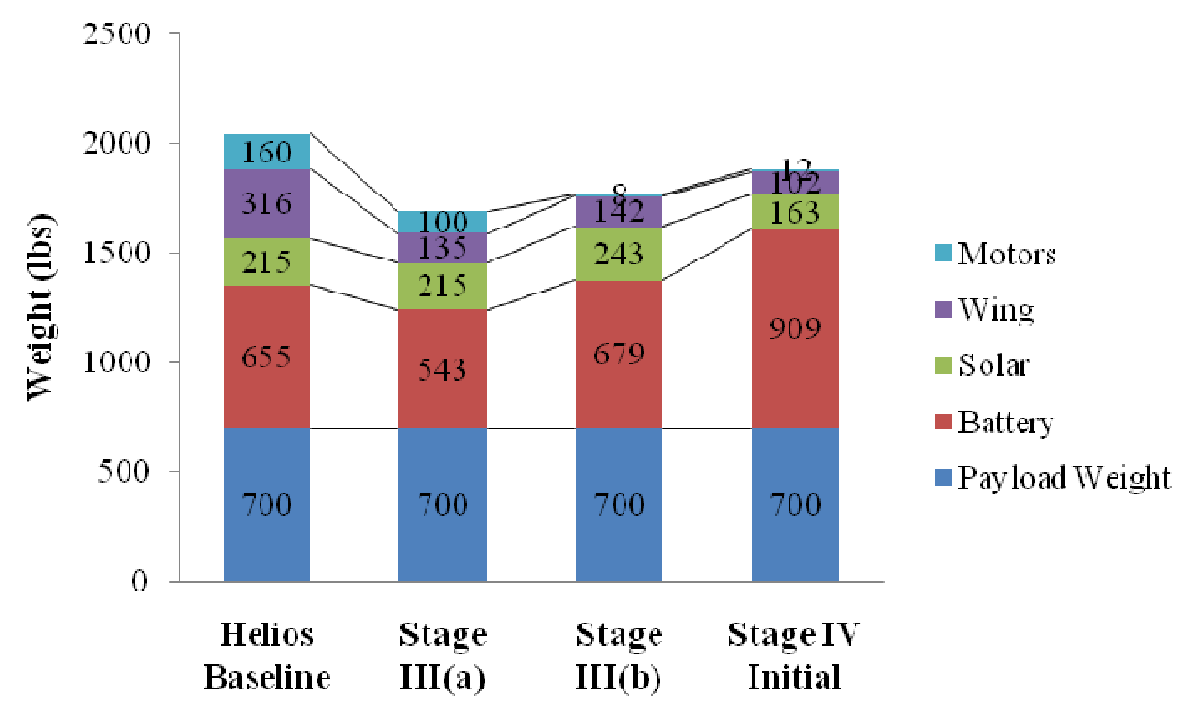

Figure 62 - Comparison of Stage IV Weight to Previous Optima

Logically it seems that we should be able to take the optimum aircraft from the previous stage, remove a portion of solar panels and have nearly the same configuration but with a decreased solar power system weight. This is a prime example of the objective function response surface stifling our optimization routine. As stated earlier, one may attempt to find the overall optimum by choosing a random selection of starting points for the process and selecting the best result among them. Table 18 presents the lowest weight configuration found from a selection ten randomized staring points. If our goal is to evaluate the benefit of implementing an ideal solar arrangement in a previously optimized configuration, we may simply select appropriate starting points. In this case, there is significant evidence that the Stage IV result in Table 18 is not actually at the global 
optimum. New start values for the design variables are selected based on the best previous configuration found for the ATLEB aircraft, and the scope of randomization for each MDO case is drastically narrowed. Table 19 shows the starting points and resulting optima for two of the best MDO runs within the focused start-value collection.

Table 19 - Focused Start Point Optimization Summary

\begin{tabular}{|l|l|l|l|l|}
\hline Design Variable & Start Point 1 & Optimum 1 & Start Point 2 & Optimum 2 \\
\hline Aspect Ratio & 36.95 & 35.61 & 37.69 & 38.76 \\
\hline Wing Loading (lb/ft ${ }^{2}$ ) & 0.77 & 0.72 & 0.82 & 0.79 \\
\hline Power to Weight (W/lb) & 7.06 & 7.07 & 7.49 & 7.02 \\
\hline Wing Thickness (\%) & 30.00 & 30.00 & 23.33 & 30.00 \\
\hline Solar Area (\% S & & 36.89 & 50.83 & 49.00 \\
\hline
\end{tabular}

The first case starts at slightly lower values of wing loading, power to weight, and aspect ratio than the second, and resulting solutions are slightly different. Despite the variance between cases 1 and 2 in Table 19, they predict aircraft weight within $1 \%$ of each other (Figure 63).

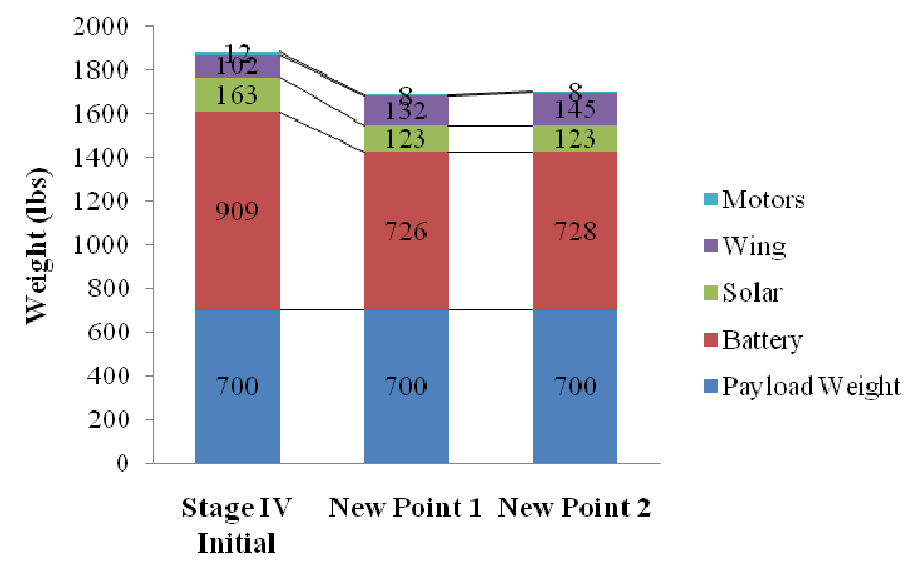

Figure 63 - Weight Breakdown for Revised Stage IV Optimization 
Because all three designs in Figure 63 represent local optima and closed designs, we may select any to move forward; weight is lowest for the first new point so "New Point 1" is chosen as the Stage IV optimum. Comparison of this case with the previous benchmark configurations is presented below. In contrast to Figure 62, we see a decrease in weight from Stage III(b) to here in Stage IV. As expected, the optimizer covers the wing with just enough solar panels to fly the mission and charge the battery during the day without wasting any power.

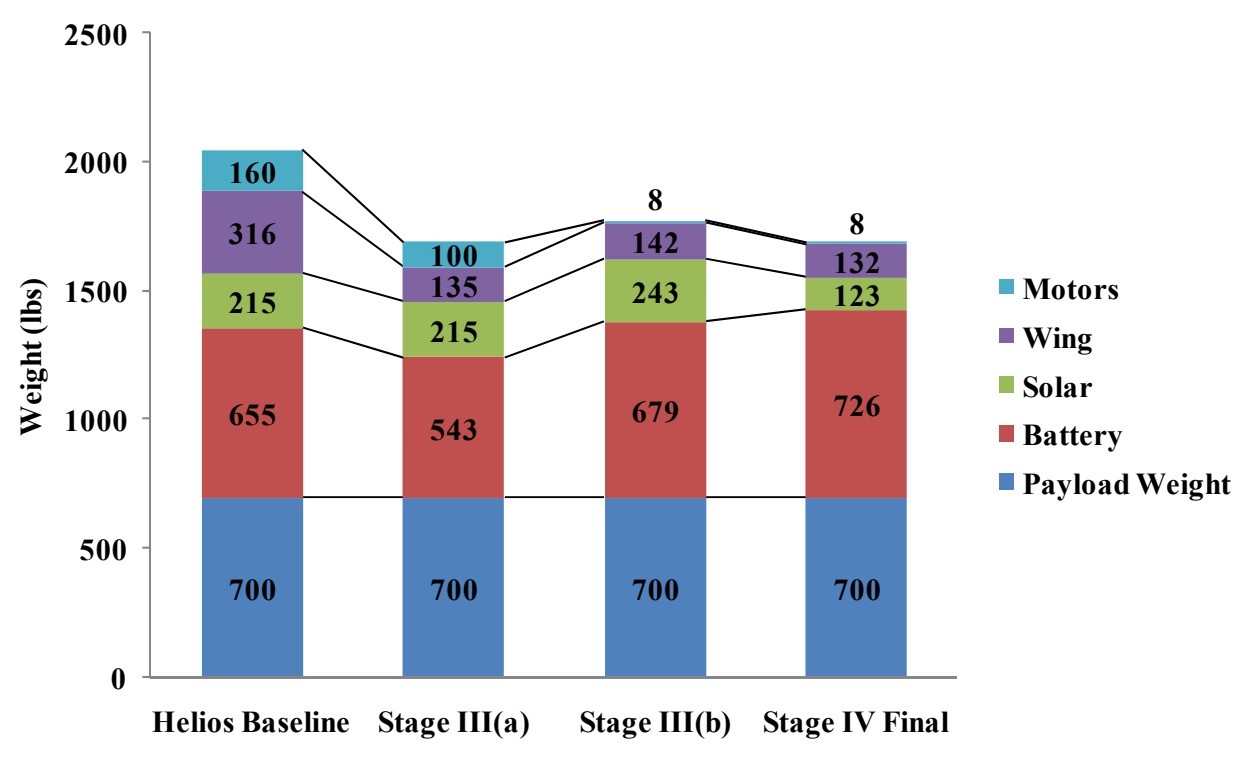

Figure 64 - Weight Comparison for Progressive Baseline Optimal Configurations

Compared to the $90 \%$ wing area coverage of all previous MDO runs, the advanced technology long endurance baseline aircraft only requires $37 \%$ of the wing area to hold solar cells. Accordingly, the weight of the solar power system is reduced by 49.3\% but there is an increase in battery weight. The advanced technology long endurance baseline aircraft has a slightly lower aspect ratio, 35.6 compared to 36.9 for Stage III(b). Similarly, the wing loading has decreased from $0.77 \mathrm{lb} / \mathrm{ft}^{2}$ at Stage III(b) to 0.72 in the current configuration. Combination of increased AR and decreased wing 99 
loading results in a higher power requirement for loiter and more battery weight needed to fly through the night. Figure 65 shows how the resulting charge profile (the red dashed line) is different from the off optimum profile. The dashed line has less "plateau time" when wasted energy is produced, but still charges the batteries enough for night flight.

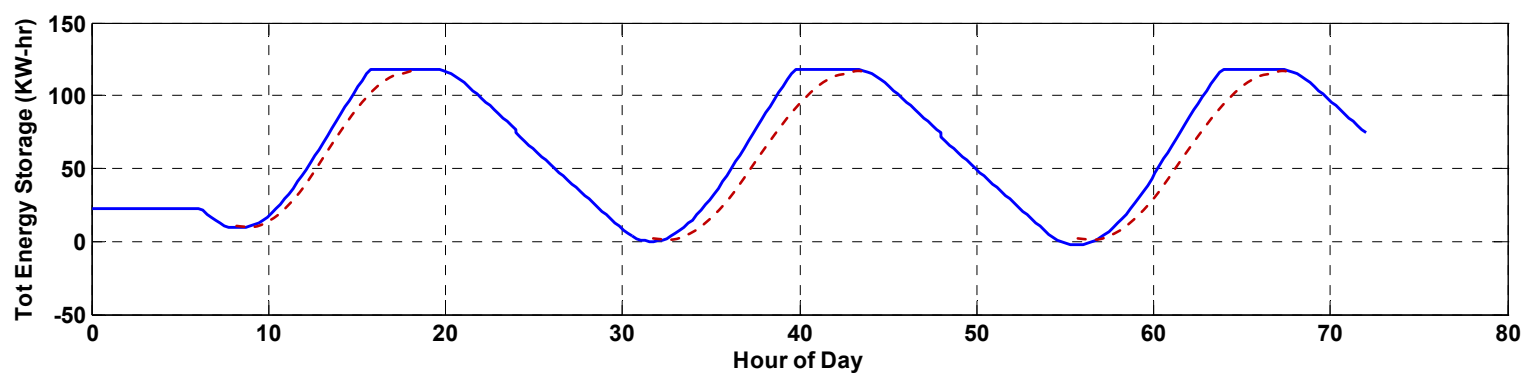

Figure 65 - Desired Battery Charge Profile

The aircraft configuration we have arrived at is considered the baseline for comparison when we examine a multiple segment wing optimized for continuous flight and using advanced technology. 


\section{Single Wing Segment Aircraft Baselines}

So far, optimal configurations have been found for two different sets of initial assumptions. First, a platform using somewhat outdated technology to perform a medium endurance mission was developed. Based on the Helios aircraft of the 1990's, this first baseline was optimized for roughly 15 hours of mission endurance, and is shown as the green aircraft in Figure 66. The second aircraft configuration used advanced technology and was optimized for multiple-day missions. Shown below in red (Figure 66) this aircraft achieves a sustainable 24-hour energy balance.

Helios MDA Sizing Result

Proven Technology Medium Endurance MDO Result

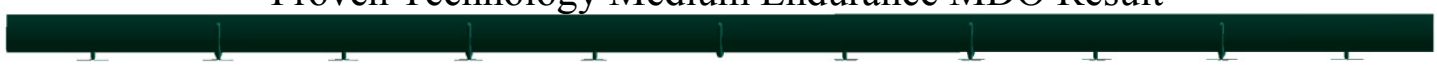

Advanced Technology Long Endurance MDO Result

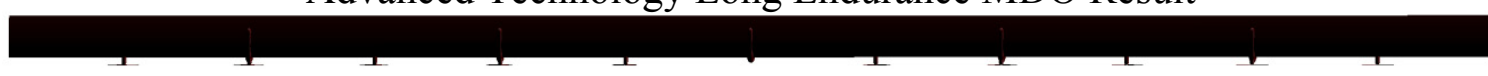

Figure 66 - Helios Proxy Alongside Two Optimal Baselines

Another perspective on these optimal baseline aircraft is included on the next page where they are compared to the entire family of ERAST vehicles (Figure 67). Note that the grey metallic aircraft in both of these figures is not simply a CAD model of Helios, but is the resulting geometry from the MDA environment when the aspect ratio, wing loading, power to weight ratio, and wing thickness are set to Helios values and the tool is tasked with sizing the aircraft. All planforms in Figure 67 are of the same scale. 
Pathfinder (1981-1997)

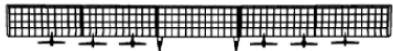

Pathfinder Plus (1997-1998)

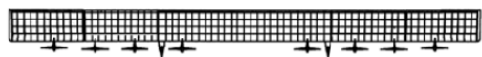

Centurion (1996-1998)

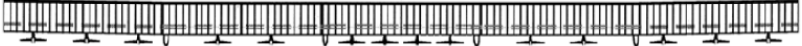

Helios Prototype (HP01), High-Altitude Configuration (1998-2002)

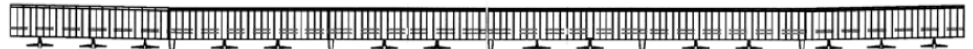

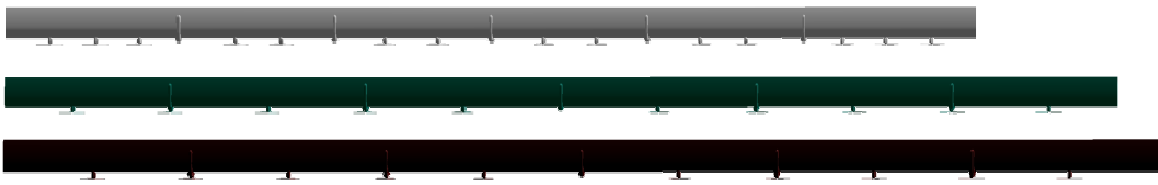

Figure 67 - Comparison of Optimized Baseline Aircraft to NASA ERAST Family

More details for each configuration are available on the following page and summarize the findings we have pointed out so far (Table 20). Despite the technological advantage of the long endurance variant, the more strenuous mission begets a lower wing loading and slightly lower aspect ratio. The two optimal configurations are quite similar, differing mainly in the area of energy storage, as the long endurance aircraft carries more batteries at a higher technology level. Also, the motor weight has decreased when implementing electric motor sizing data from [36].

Before moving to the optimization of a multiple segment planform, one last investigation will be presented. The baseline aircraft have all included a payload of 700 pounds that draws $2 \mathrm{~kW}$ constantly over a 24 hour period. It is prudent to evaluate how the MDO tool responds to changing these payload requirements, because HALE missions may vary largely is this respect. The following section addresses this issue for the ATLEB aircraft. 
Table 20 - Baseline Aircraft Summary

\begin{tabular}{|c|c|c|c|}
\hline & & PTMEB & ATLEB \\
\hline Gross Weight & lbs & 1777 & 1778 \\
\hline Aspect Ratio & & 37.62 & 35.61 \\
\hline Wing Loading & $\mathrm{lb} / \mathrm{ft}^{2}$ & 0.83 & 0.72 \\
\hline Power to Weight & Watt/lb & 7.28 & 7.07 \\
\hline Wingspan & $\mathrm{ft}$ & 284.43 & 295.64 \\
\hline Chord & $\mathrm{ft}$ & 7.56 & 8.30 \\
\hline $\mathbf{S}_{\text {ref }}$ & $\mathrm{ft}^{2}$ & 2150.66 & 2454.59 \\
\hline Wing Thickness & & $30 \%$ & $30 \%$ \\
\hline $\mathrm{C}_{\text {Lmax }}$ & & 1.5 & 1.5 \\
\hline Max Climb Rate & fpm & 234.4 & 227.7 \\
\hline Sea Level $V_{\text {stall }}$ & $\mathrm{ft} / \mathrm{s}$ & 21.5 & 20.2 \\
\hline Cruise Dash & $\mathrm{ft} / \mathrm{s}$ & 120.5 & 126.4 \\
\hline Battery Energy & $\mathrm{kW}-\mathrm{hr}$ & 22.5 & 131.8 \\
\hline Payload Weight & $\mathrm{lb}$ & 700 & 700 \\
\hline Payload Power & $\mathrm{kW}$ & 2 & 2 \\
\hline Battery Weight & $\mathrm{lb}$ & 542.8 & 726.3 \\
\hline Solar Weight & $\mathrm{lb}$ & 214.8 & 122.7 \\
\hline Wing Weight & $\mathrm{lb}$ & 134.7 & 132.3 \\
\hline Motor Weight & $\mathrm{lb}$ & 100.0 & 7.9 \\
\hline
\end{tabular}




\section{Different Payloads: Weight and Power Requirement}

The initial payload of 700 pounds and $2 \mathrm{~kW}$ was chosen to represent the upper echelon of payload weight that Helios claims to support, with a power requirement intended to envelope several current or past HALE mission concepts. Payload requirements for the communications platform design challenge posed by the AIAA were 440 pounds that draw $1.5 \mathrm{~kW}$ [8]. The Turin Polytechnic University in Turin, Italy proposed a design supporting a 220 pound $1.3 \mathrm{~kW}$ payload [47]. Also, a study of alternatives and technology requirements for HALE UAVs proposed two separate missions, a communications payload of 440 pounds drawing $1.5 \mathrm{~kW}$, and a hurricane science mission supporting an 880 pound, $2.5 \mathrm{~kW}$ payload [49].

Rather than analyzing each of these cases individually, the payload requirement is perturbed both in weight and power on both sides of the current ATLEB values. Figure 68 shows the total weight breakdown of resulting optimal aircraft carrying different payload weights. The actual parametric change in aircraft weight is skewed by the substantial payload contribution, so Figure 69 presents just the dependant components.

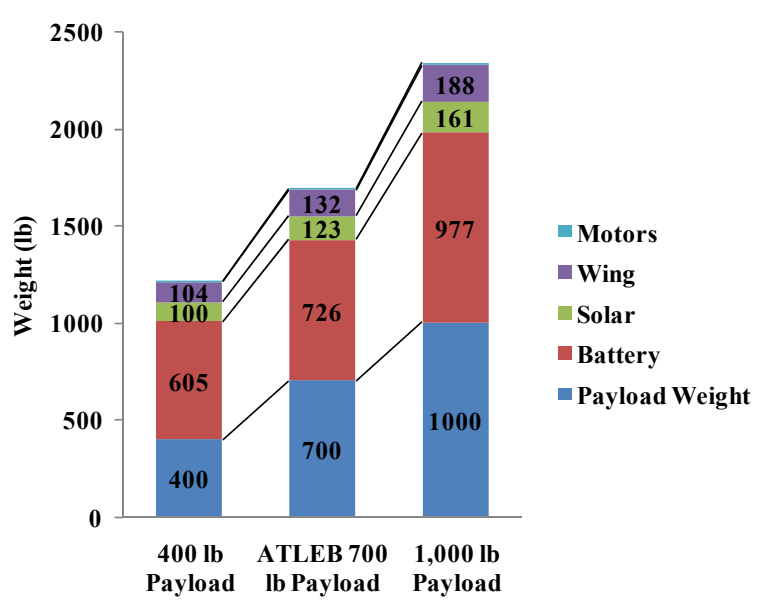

Figure 68 - Weight Breakdown for Different Payload Weights 


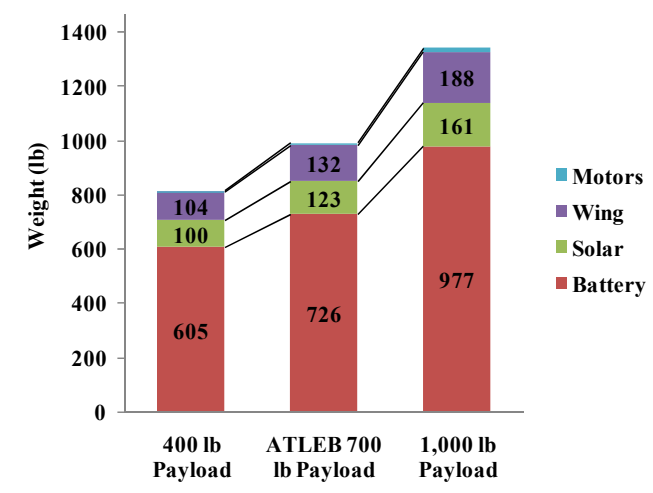

Figure 69 - Battery, Solar, and Wing Weight Sensitivity to Payload

In general we expect to see the aircraft component weights increasing to accommodate a larger payload. Specifics about the resulting configurations are presented shortly. The second aspect of electric aircraft payload requirements is the power needed. We return to the baseline payload of 700 pounds and $2 \mathrm{~kW}$ to compare aircraft configurations with varying payload power (Figure 70).

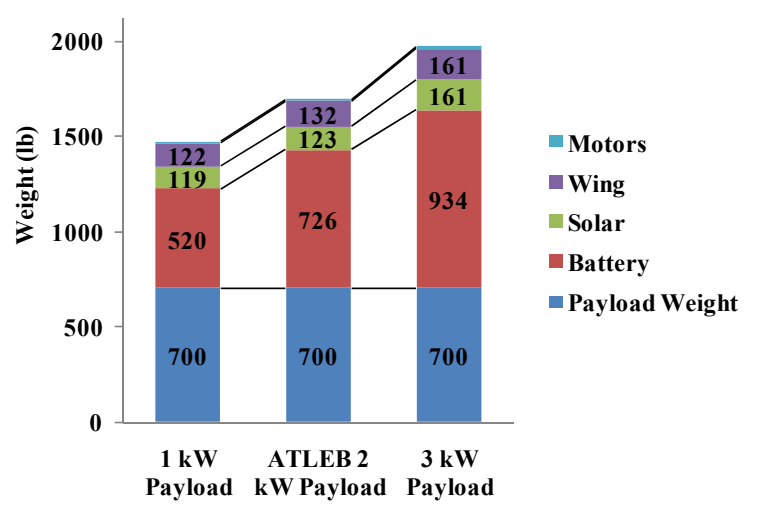

Figure 70 - Weight Breakdown for Different Payload Power

Changing the power of the payload by $1 \mathrm{~kW}$ either way seems to affect almost exclusively the battery weight. One important note is that if the CONOPS is modified such that the payload is not in operation during the night, the effect of changing the power requirement is much less, and will simply require more solar coverage of the wing to power mission operations and supply regenerative energy. 
Configuration parameters are summarized in Table 21 below. Several points to notice are that for a heavy or high power payload, the coverage of wing area by solar cells has increased to roughly $60 \%$. Also, the planforms for the aircraft are essentially the same, with aspect ratio, wing span, and chord falling close enough to attribute differences to loose optimization tolerances. What this means is that if an aircraft is designed with some margin in the spar to account for the slight changes between Figure 69 and Figure 70 , the same platform may be used for different payloads. All that would be necessary would be refitting more or less batteries and reconfiguring the solar panels.

Table 21 - Payload Study Optima Comparison

\begin{tabular}{|l|l|l|l|l|l|l|}
\hline \multirow{2}{*}{} & \multirow{2}{*}{ ATLEB } & \multicolumn{5}{|c|}{ Payload } \\
\cline { 4 - 7 } & & & Low Pow. & \multicolumn{1}{|c|}{ Light } & High Pow. & Heavy \\
\hline Gross Weight & $\mathrm{lbs}$ & 1778 & 1546 & 1277 & 2071 & 2463 \\
\hline Wing Loading & $\mathrm{lb} / \mathrm{ft}^{2}$ & 0.72 & 0.64 & 0.54 & 0.88 & 1.01 \\
\hline Power to Weight & Watt/lb & 7.07 & 6.68 & 6.04 & 7.46 & 8.03 \\
\hline Wing Thickness & $\%$ & 30.0 & 29.4 & 30.0 & 30.0 & 30.0 \\
\hline Solar Area & $\%$ & 36.9 & 44.3 & 38.1 & 62.0 & 60.0 \\
\hline Wingspan & $\mathrm{ft}$ & 295.64 & 300.0 & 300.0 & 297.0 & 300.0 \\
\hline Chord & $\mathrm{ft}$ & 8.30 & 8.10 & 7.90 & 7.90 & 8.14 \\
\hline
\end{tabular}

With these baselines defined, we now move into the optimization of a multiple segment wing configuration. Unlike the process of this chapter, the optimization of a multi-segment wing will not proceed in stages, but begins by immediately including all of the design variables discussed thus far. 


\section{SEGMENTED WING OPTIMIZATION}

The MDO tool created for this research was built to allow the user to input the number of separate pin-connected wing segments, automatically modifying the analytical processes accordingly. All results presented up to this point have designated one wing segment, and have performed the MDA on a flying wing span loaded architecture. As we progress now thorough the investigation of multiple wing segments, the same MDA environment is used, and built-in contingencies allow the same utilization process as single segment baseline studies.

Initially, it would seem desirable to simply allow the MDO tool to find the optimum number of wing segments, just as it would any other design variable. Methods of Integer Programming do exist to solve optimization problems with discrete variables [29]. The implementation of Integer Programming in MATLAB is the function bintprog found in the Optimization Toolbox. Technically, the problem at hand falls under the category of Mixed Integer Programming, where the set of design variables is composed of both continuous and discrete entries [29]. Unfortunately, bintprog solves only Binary Integer Programming problems, and requires the solution to take the form of a binary integer vector. Since the possible values for number of pinned wing segments represents a relatively small set, rather than implementing a custom Mixed Integer Programming optimization routine, we simply evaluate optimal solutions for each pinned wing configuration. What follows is a discussion of the optimization results for increasing numbers of pinned wing sections, starting with the span divided in half for a two-segment platform. 


\section{Two Segment Wing}

Here, we have divided the flying wing into two segments, with a pinned joint at the aircraft centerline. The MDO tool is initialized with the same technology and mission parameters as the Advanced Technology Long Endurance Baseline aircraft, and similar to earlier optimizations, a variety of cases are run to increase the probability of finding a global optimum. Results for one of the more attractive optima are included below (Table 22).

Table 22 - Two Segment Wing Optimization Summary

\begin{tabular}{|l|l|l|l|l|}
\hline Design Variable & Start Point & Lower Bound & Upper Bound & Optimum \\
\hline Aspect Ratio & 37.06 & 10 & 60 & 37.16 \\
\hline Wing Loading (lb/ft' $\left.{ }^{2}\right)$ & 0.82 & 0.5 & 5.0 & 0.67 \\
\hline Power to Weight (W/lb) & 10.77 & 1.0 & 60.0 & 6.35 \\
\hline Wing Thickness (\%) & 10.23 & 8.0 & 30.0 & 15.18 \\
\hline Solar Area (\% S Sef) & 53.88 & 0.0 & 98.0 & 39.51 \\
\hline
\end{tabular}

Several interesting trends of this configuration compared to the baseline aircraft are immediately visible. The optimal aspect ratio of the two-segment aircraft as increased by $4.3 \%$, wing loading has decreased $7.3 \%$, power to weight ratio has decreased $10.2 \%$, the wing thickness no longer runs to its upper limit, and solar coverage of the wing has decreased from $45 \%$ to $39.5 \%$. Tracking the objective function value and constraint violation through the optimization shows that the optimizer reached a feasible point after 18 iterations, and was not able to substantially improve the solution past that point (Figure 71). Despite the lack of improvement after satisfying all constraints, this solution 
represents the best of 58 separate optimization processes, and gives a gross weight of 1,627 pounds, an $8.5 \%$ improvement over the baseline optimum.

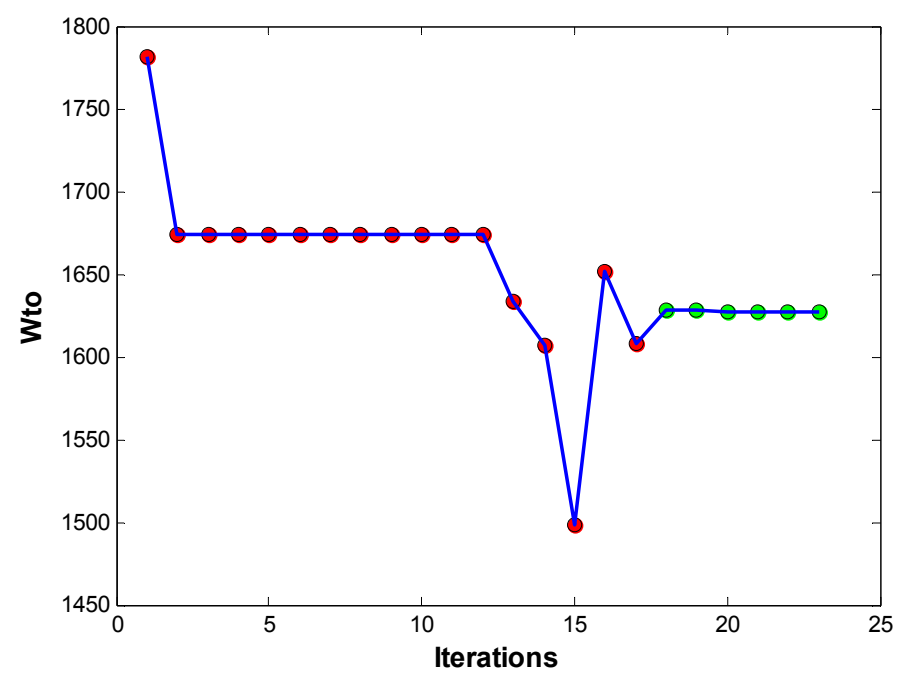

Figure 71 - Two Segment Objective Function History

Because the two-segment optimum platform has seen a decrease in wing loading and an increase in aspect ratio over the baseline, loiter and cruise power requirements decrease, allowing a reduction in battery energy storage for night flight, corresponding to a reduced battery weight. Figure 72 compares this optimum with the baseline long endurance aircraft by weight breakdown, illustrating the $8.3 \%$ battery weight reduction. Another major contributor to the differences between this configuration and the baseline is the structural weight of the wing, decreasing for the segmented configuration as expected because we now have essentially two sub-aircraft each with half the aspect ratio of the overall planform. Decreasing the effective AR for the sub-aircraft provides a $50 \%$ reduction is wing weight. As seen in previous cases, the two-segment aircraft optimum lies on the wingspan constraint of $300 \mathrm{ft}$. Compared to the baseline, the aircraft here has nearly the same reference area, differing only $1.4 \%$ because its wingspan is only $295 \mathrm{ft}$ and it has a larger chord than the two-segment optimum. Now, because of our reduced 109 
battery requirement, the solar area coverage may be decreased. Accordingly, with a similar wing area but having $5.5 \%$ fewer solar cells, a decrease in solar power system weight is observed.

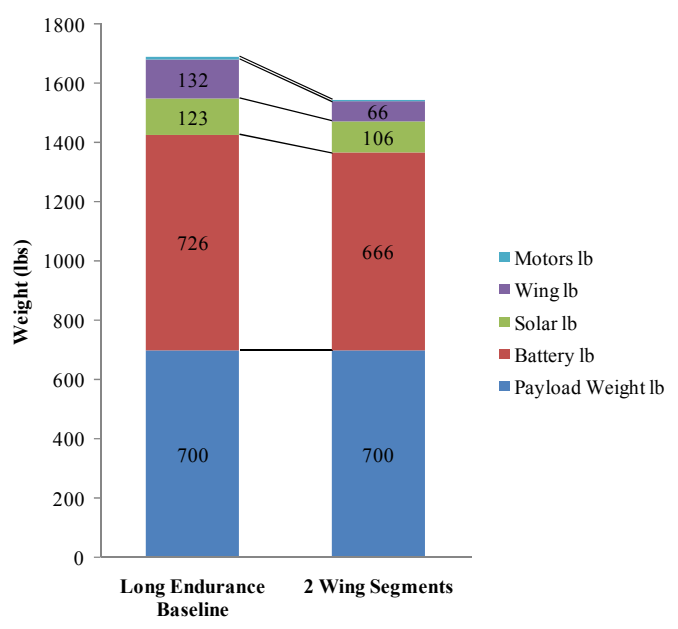

Figure 72 - Weight Comparison for Two Segment Aircraft

We have pointed out several times that the non-smooth nature of the objective function response surface encourages multiple optimization runs from different starting points. From the set of solutions, the best candidate may be chosen, and the result in Table 22 and Figure 72 is one such selection. When making a selection in this manner, it is prudent to examine the results and trends to provide some background understanding of the design space. Figure 73 is a matrix of plots depicting all of the MDO solutions for each individual design variable compared to every other. The plots show the starting points as hollow circles, illustrating the Monte Carlo style in which they are randomly distributed over some domain. Solution points are the filled points, and are colored according to the resulting gross weight of that specific case. For Figure 73, the lowest weight points are colored blue and are more or less clustered in some fashion. There are some outliers and diverging points, but in general, our choice of a global optimum 
(designated by a star outlined in magenta) is surrounded closely by other low weight points. For a well behaved monotonic objective function with one global minimum, all of the filled points would fall on top of one another, clearly illustrating the existence of local minima in our HALE MDO environment. One possible cause of this behavior is the loosening of convergence tolerances to decrease run time. Loose tolerances coupled with a response surface that is somewhat flat near the optimum as evidenced by the somewhat wide clustering of points with similar weight values in Figure 73 will invariably result in the variety of solutions that we see.
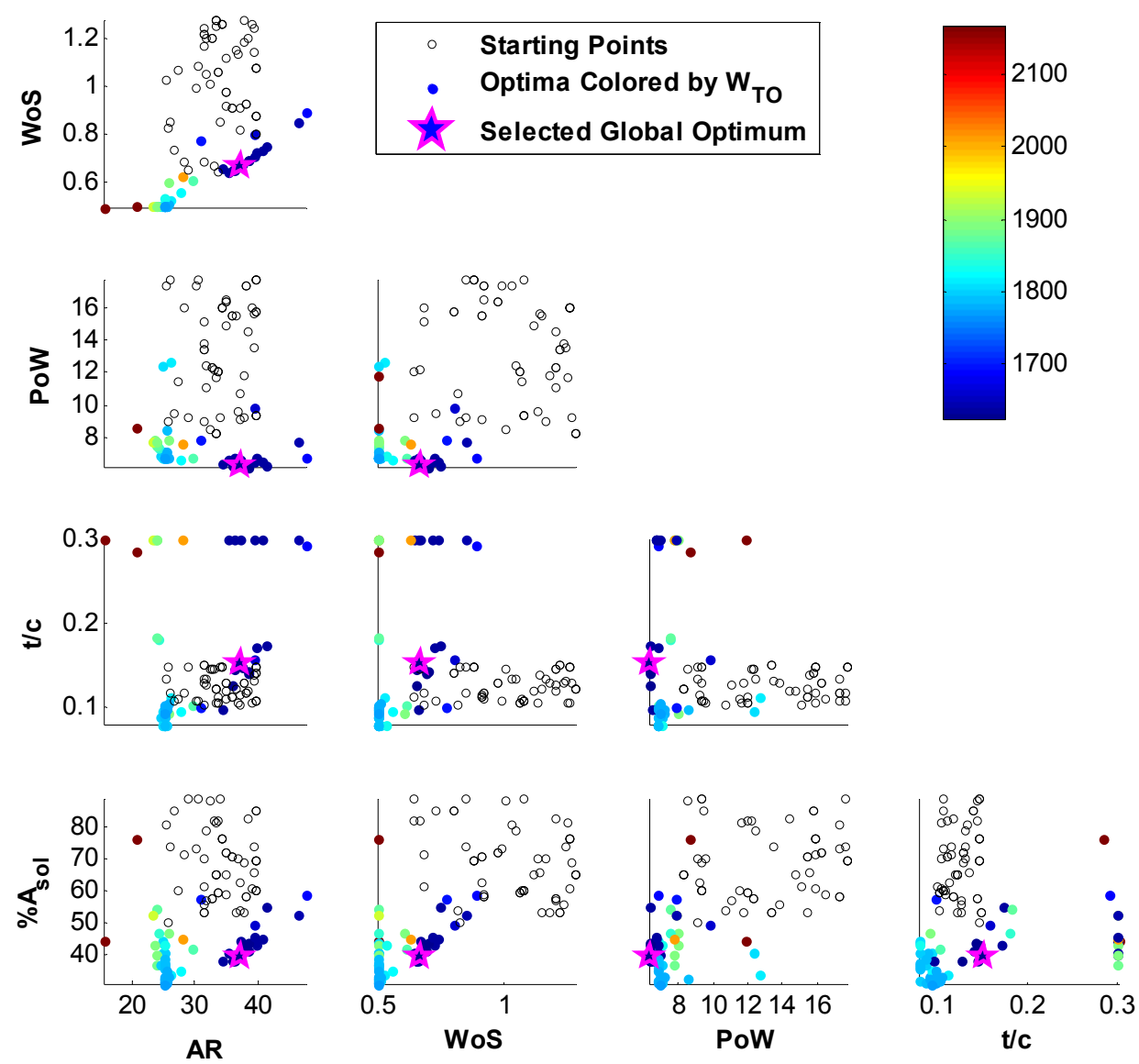

Figure 73 - Plot Matrix of All Optimization Cases for a Two Segment Platform 
Several other methods for visualizing the optimization results and trends highlight some of the interesting behaviors of the design space. Figure 74 is a scatter plot showing the gross weight of the solution against wing loading. Points in Figure 74 are colored by aspect ratio. This plot illustrates a division among the solutions into two regions. Solutions clustered at the low wing loading value have run into the lower bound imposed on wing loading, and exhibit a trend of lower aspect ratios than the other solution cluster. In the area surrounding the selected global optimum, solutions are of lower gross weight, higher wing loading, and higher aspect ratio. Though a higher aspect ratio in general will increase the wing weight, our gross weight is dominated by payload weight (a constant for all points) and battery weight. What we see then, is that the benefits from lower required power and decreased battery weight from the high aspect ratio wing outweigh the structural penalty.

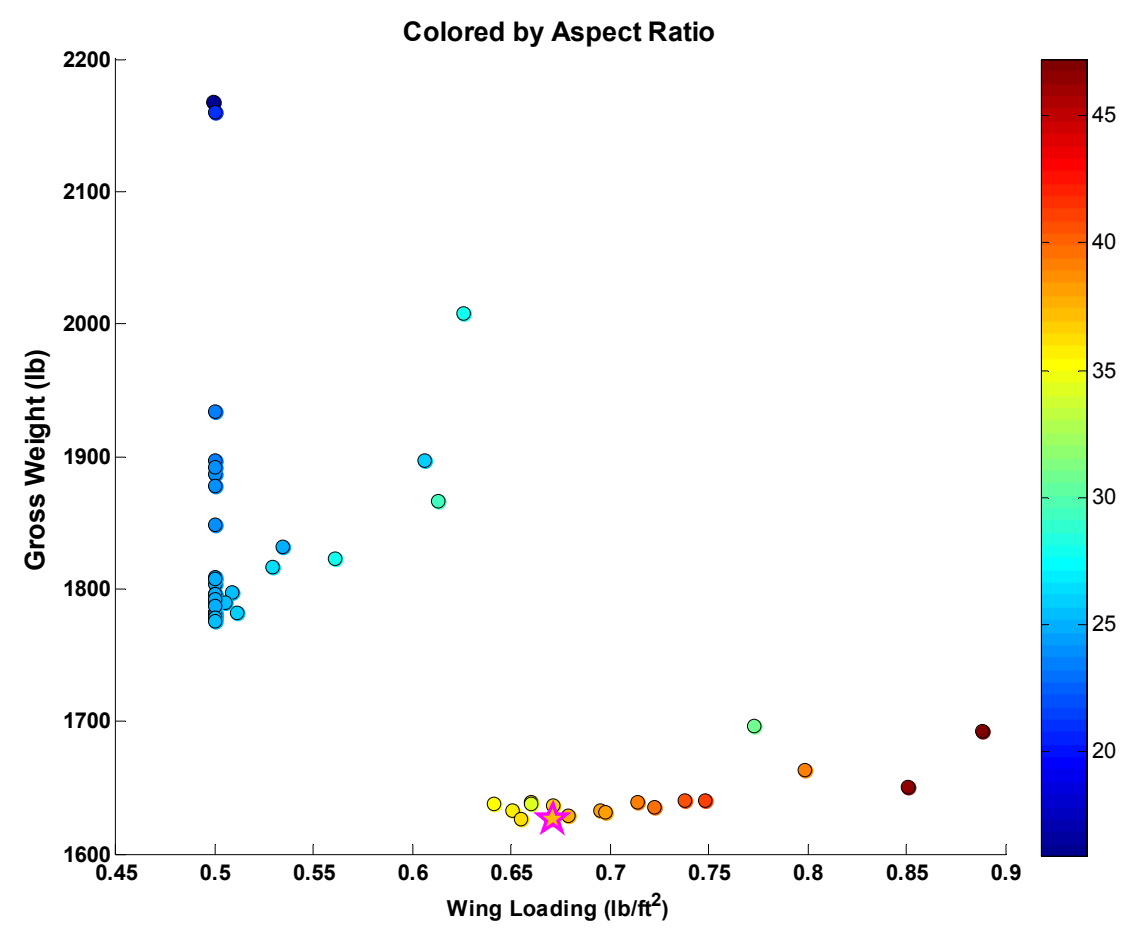

Figure 74 - Two Segment MDO Solution Space 
Another illustration of this bifurcation of the solutions is presented with the set of graphs in Figure 75. Here, the solutions are sorted in order of increasing gross weight, and colored according to various aircraft characteristics, reiterating several clear trends. First, the solutions that converge to 1800 pounds (the higher weight) all show an aspect ratio of 25 , a wing loading of 0.5 , and the lower bound on wing thickness.
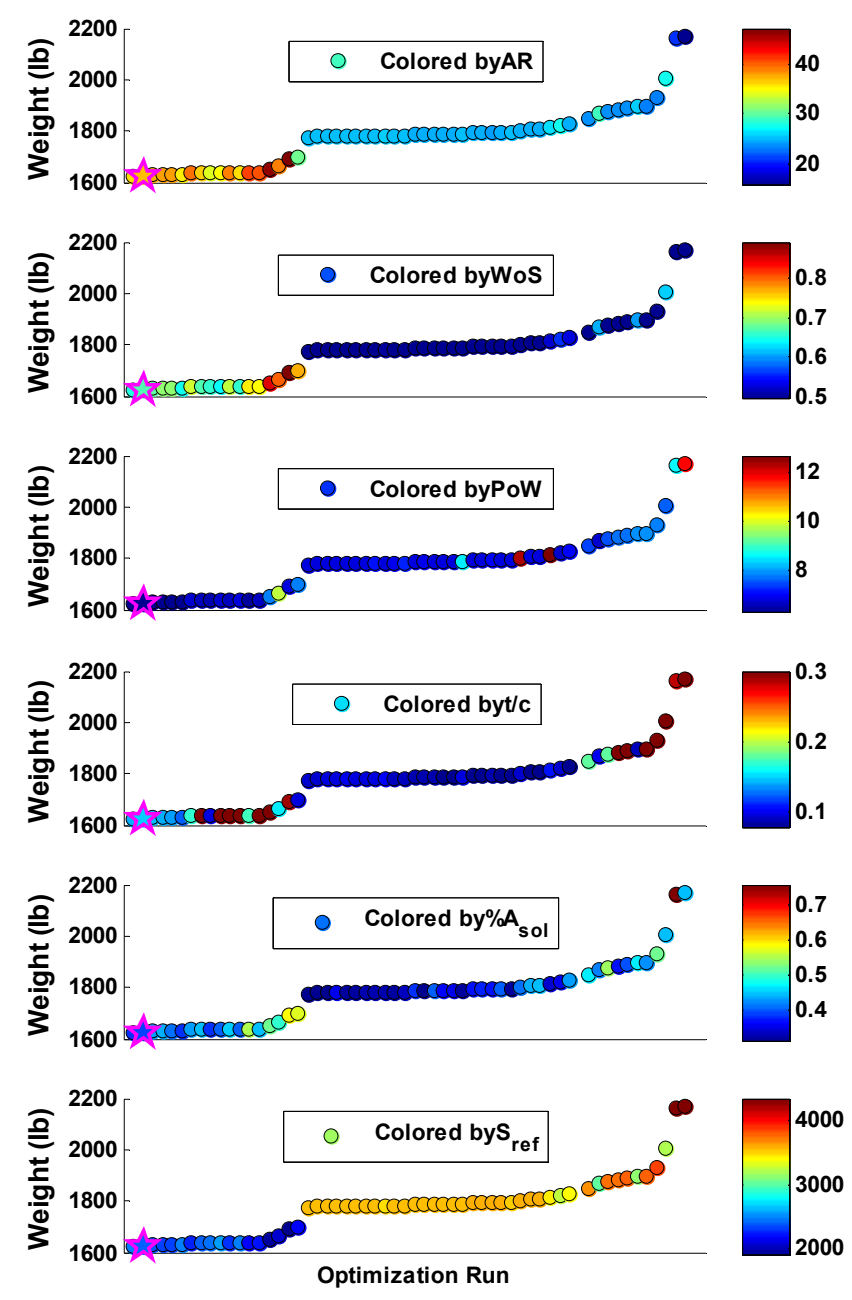

Figure 75 - Two Segment Solutions Sorted by Gross Weight

Delving deeper into these solutions, we find that one of the major discriminators between the low weight plateau and the higher weight group is the battery charge profile. One reason that two groups of solutions exist is that the optimization environment fails to 
sufficiently minimize solar coverage in the high weight cases. The resulting charge profiles resemble Figure 30 with plateaus of stored energy. This, however, does not fully explain what is happening. If we look at the design variable history for a variety of cases in the lower cluster compared to those of higher weight, another trend emerges. It appears that the search direction for the second iteration causes the wing loading variable to run into its lower bound and remain there for the rest of the optimization. Three examples are shown below (Figure 76).
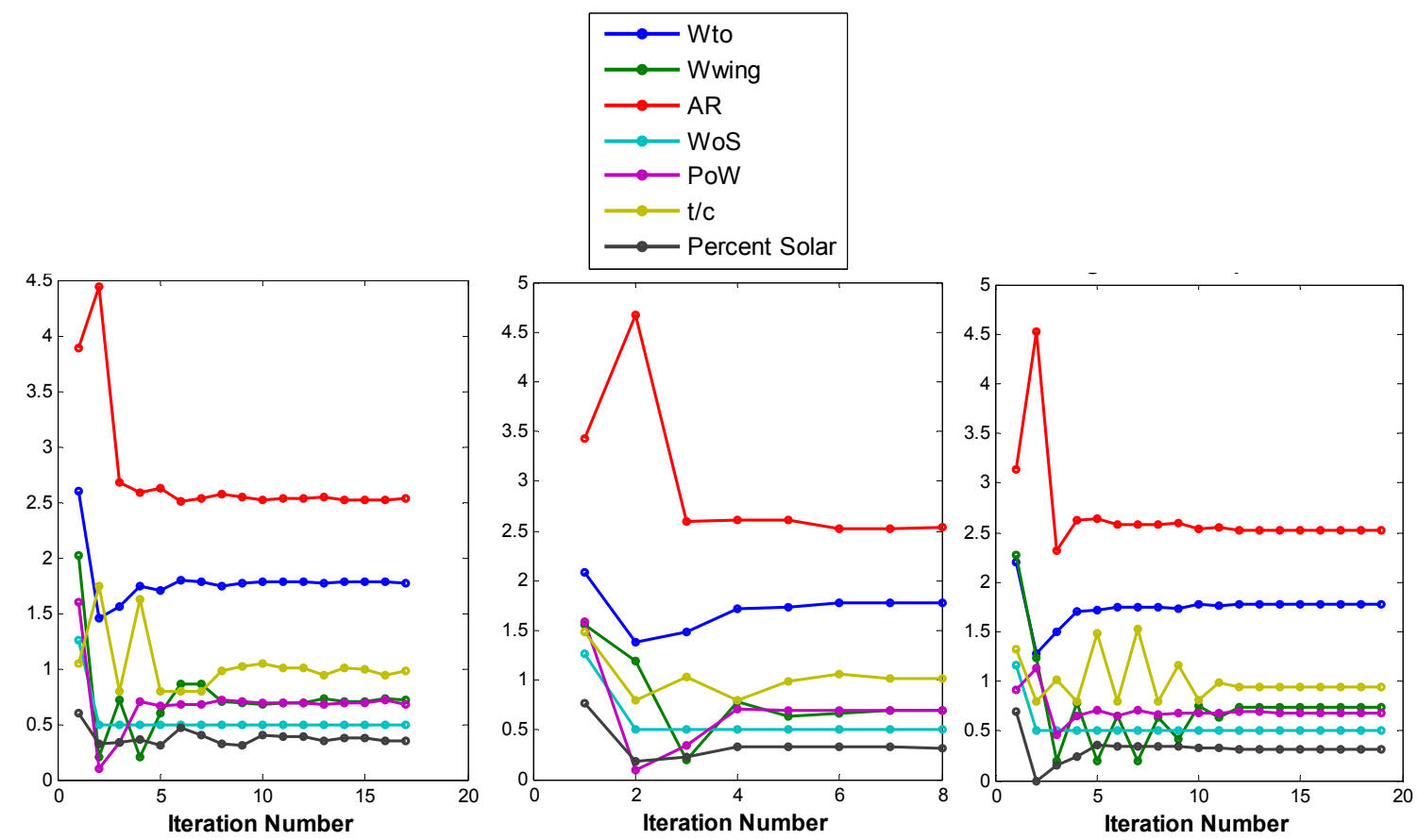

Figure 76 - Design Variable History for Anomalous Solutions

This behavior is in contrast to that seen with the lower weight solutions, shown in Figure 79. Here we see that the wing loading value (teal) may hit the lower bound, but does not remain at the same value throughout the optimization. Table 23 summarizes the solution clusters, providing the average for each group. 

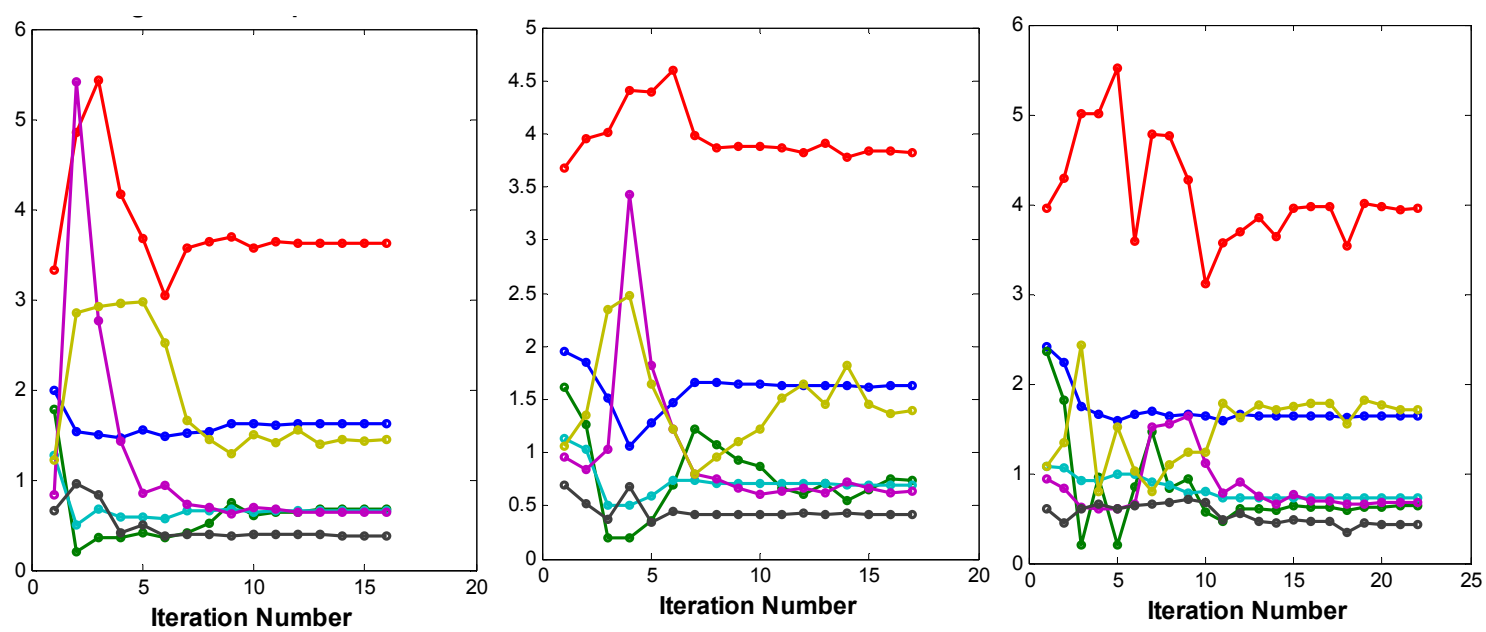

Figure 77 - Design Variable History for Attractive Solutions

Table 23 - Average Values for Solution Clusters

\begin{tabular}{|l|l|l|l|l|}
\hline \multirow{2}{*}{ Aspect Ratio } & \multicolumn{2}{|l|}{ Low Weight Group } & \multicolumn{2}{l|}{ High Weight Group } \\
\cline { 2 - 5 } & Mean & Std. Dev. & Mean & Std. Dev. \\
\hline Wing Loading (lb/ft ${ }^{2}$ ) & 38.3 & 3.92 & 25.3 & 1.05 \\
\hline Power to Weight (W/lb) & 0.72 & 0.07 & 0.51 & 0.03 \\
\hline Wing Thickness (\%) & 20.2 & 8.14 & 11.4 & 6.19 \\
\hline Solar Area (\% S S & & 0.87 & 7.4 & 1.36 \\
\hline
\end{tabular}

The root cause of the difference between the two solution clusters, however, has been traced to the aerodynamics module, and the use of EDET as a drag code. Currently, the implementation of EDET does not model a continuously changing laminar to turbulent transition point on the wing. Rather, the code flags either a fully turbulent or a fully laminar boundary layer based on whether the Reynolds' Number based on chord is below or above 500,000 . This sort of discrete change in a variable or quantity, especially one as central as the drag coefficient, will definitely cause problems for our optimizer, and in this case, results in a divided solution space. Figure 78 shows that the cluster of 
low weight solutions, because of their higher wing loading and aspect ratio, are below the cutoff for turbulent boundary layer friction coefficient equations, and are assumed to be laminar.

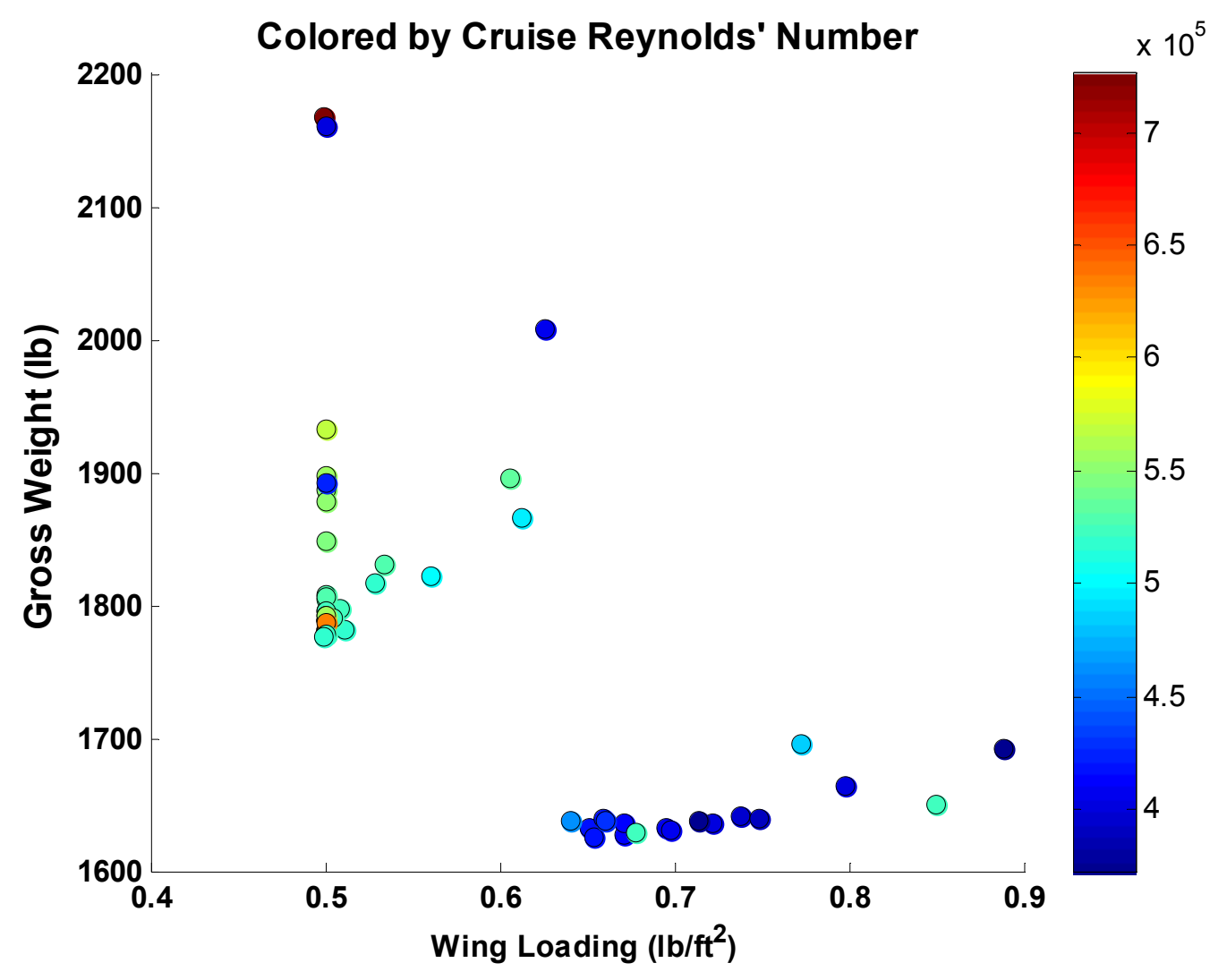

Figure 78 - Two Segment Solutions: Reynolds' Number

Because the goal for these MDO processes was minimization of gross weight, we focus on the low weight cluster when choosing the optimal two segment configuration. Indeed, all of the points in Figure 73, Figure 74, and Figure 75 are optima in the sense that they have met the constraints imposed and the optimizer has found the minimum weight for some tolerance. We therefore have selected the point shown as a star in the figures and described in Table 22 to represent the best design of this architecture. Mean values from Table 23 are presented only for illustrative purposes. 


\section{Additional Wing Segments}

The same procedures described for the two segment wing are used to evaluate any number of pinned wing segments. Rather than presenting specific results one at a time for each case, results are discussed here comparing the set of two to six wing segments. Optimum configurations for the various pinned wing platforms are all selected as the minimum weight solution from a large set of optimization runs. The weight breakdowns for the best solutions are compared in Figure 79.

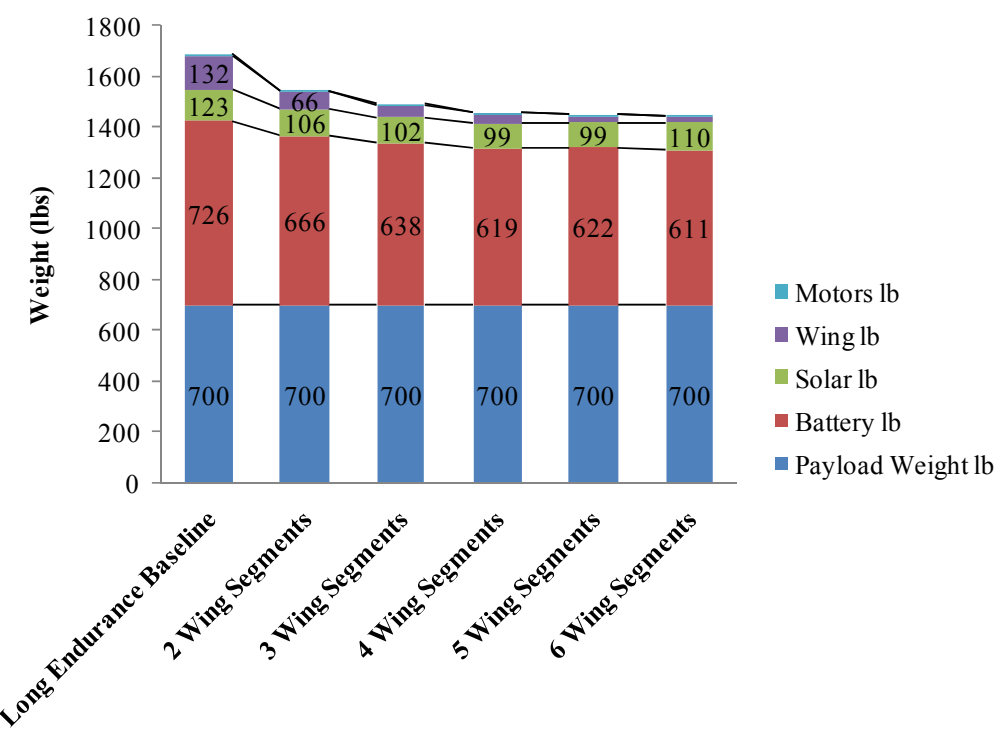

Figure 79 - Weight Breakdown Comparison of Multiple Wing Segment Configurations

The most noticeable aspect of this chart is that with an increasing number of wing segments, the weight of the optimal aircraft converges to similar values and benefits of additional segments disappear. Of all the parameters and characteristics of each optimal aircraft configuration, one of the more interesting ones to explore when looking at increasing the number of wing segments is the wing thickness. We expected that 
increasing the number of segments would decrease the structural loading on the spar, and Figure 79 confirms this. What happens with the higher number of segments is that the wall thickness of the spar that is required to support the loads decreases to the minimum allowable value of 0.005 inches. Earlier, it was shown that the aerodynamic analysis process is sensitive to wing thickness (Figure 27) and higher thickness results in a higher $\mathrm{C}_{\mathrm{Do}}$. Since wing thickness is a design variable, and spar wall thickness is constrained by a lower bound, the optimizer decreases the wing thickness to chord ratio to reduce power required, battery weight, solar weight, etc. A thinner wing results in a smaller spar cross section and increased principal stresses, so we imagine that there exists a Pareto front of low spar weight and low wing thickness. One segment designs showed a tendency toward high thickness because the aerodynamic benefits of a thin wing were outweighed by the large increases in structural weight. Figure 80 tracks the wing thickness for different numbers of wing segments, and we see the upper bound of 30\% thickness to chord ratio for a single segment wing. Also apparent is the ability of the optimizer, when analyzing a multiple segment configuration, to decrease the thickness some amount before seeing the structural penalty. Compared to the single segment, $t / c$ is halved for two segments.

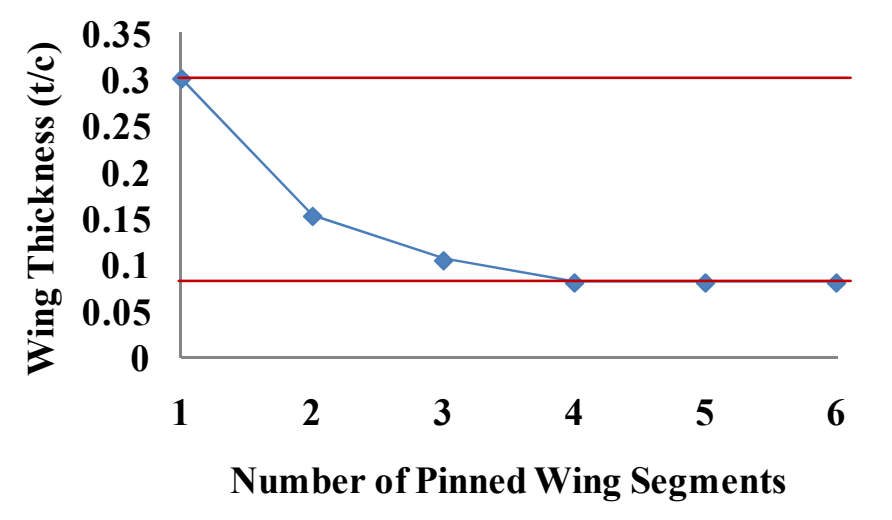

Figure 80 - Optimal Wing Thickness for Multiple Wing Segments 
Next, we look back to Table 22 and have represented the upper and lower bounds for the thickness to chord design variable as red lines on Figure 80. It seems that for more wing segments, the lower structural loads facilitate thinner and thinner wings, eventually encountering the lower bound on the variable. Keep in mind that Figure 80 actually represents the wing thickness as a percent of chord length, and that we haven't quoted any actual maximum thickness values. This means that the optimizer may have arrived at a minimum thickness to chord ratio, but can still further decrease the actual thickness value by decreasing the chord. Figure 81 shows that if the aircraft has more than 4 wing segments, there is a sharp decline in chord length, and with the wingspan at the upper limit of $300 \mathrm{ft}$ for all cases, this translates directly to a sharp decrease in $\mathrm{S}_{\text {ref }}$ (Figure 82).

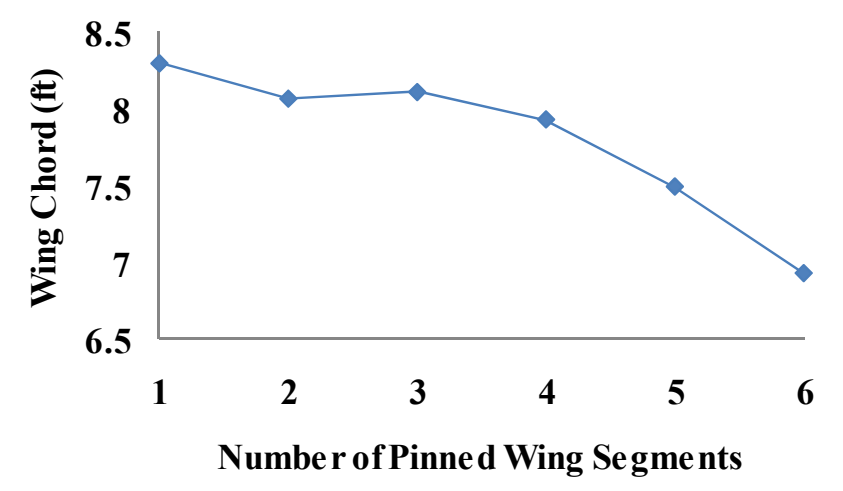

Figure 81 - Optimal Wing Chord for Multiple Wing Segments

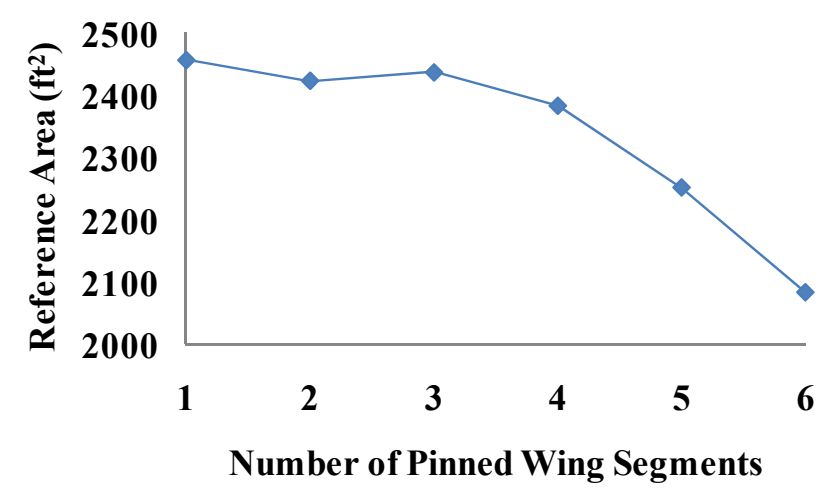

Figure 82 - Optimal $S_{\text {ref }}$ for Multiple Wing Segments 
Another result of the change lower bound on thickness to chord ratio becoming active is apparent in the percent of the wing requiring coverage. The actual area of solar cells for each case is generally similar, so decreasing the wing area simply means that a greater percent of the wing must be covered (Figure 83).

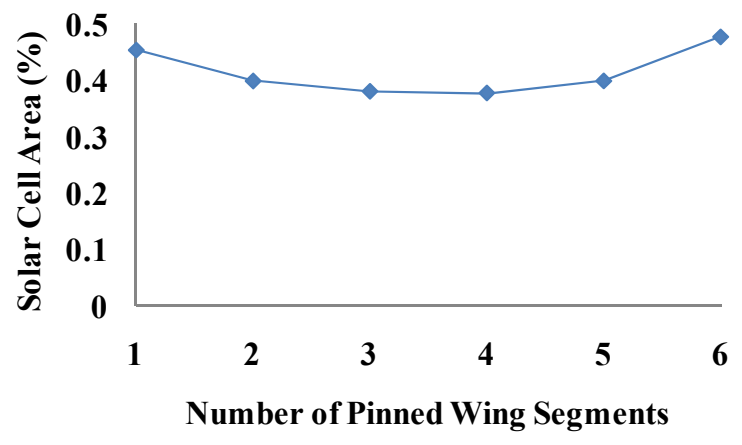

Figure 83 - Optimal Solar Coverage (Percent)

While Figure 79 shows slight improvements in structural weight between 3, 4, and 5 segments, the reality is that the gross weight of the aircraft is nearly the same (Figure 84). When reference area is decreased for an aircraft with a given weight, the result is a higher wing loading, a result that we see in Figure 85.

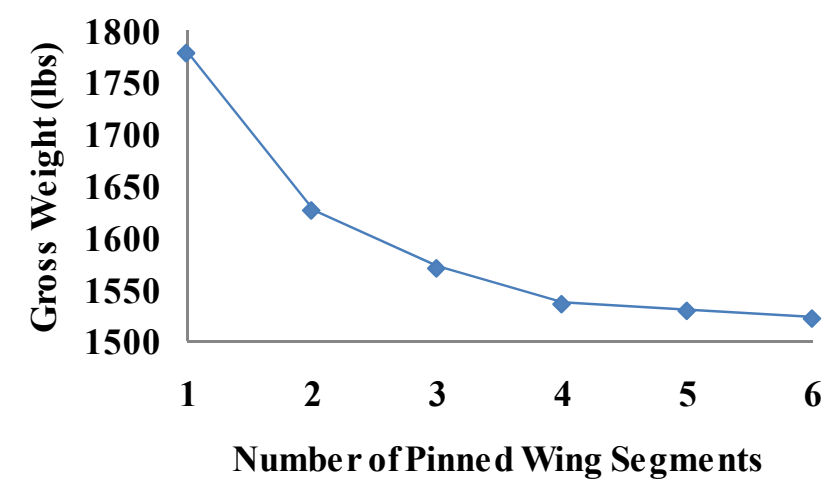

Figure 84 - Optimal Gross Weight for Multiple Wing Segments 120 


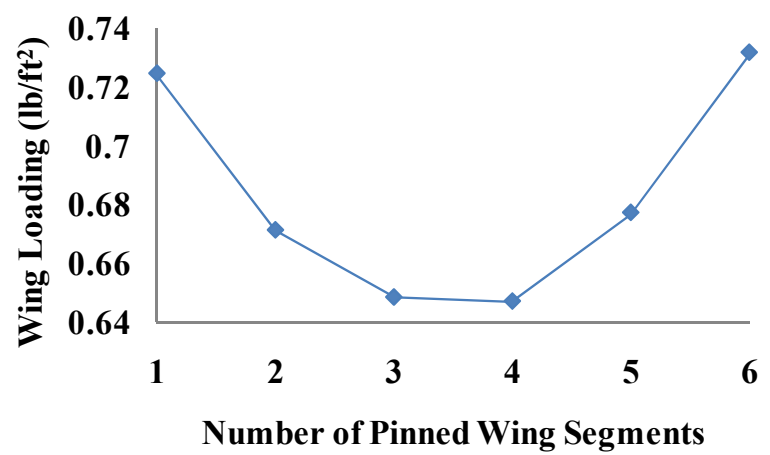

Figure 85 - Optimal Wing Loading for Multiple Wing Segments

We might expect that higher wing loading would increase required power, battery weights, etc, but the results in Figure 79 and Figure 84 do not show this happening. In fact, the optimizer has increased the aspect ratio, made possible within the wingspan constraint because of the decreased chord length for higher numbers of wing segments. Figure 86 tracks aspect ratio for each configuration.

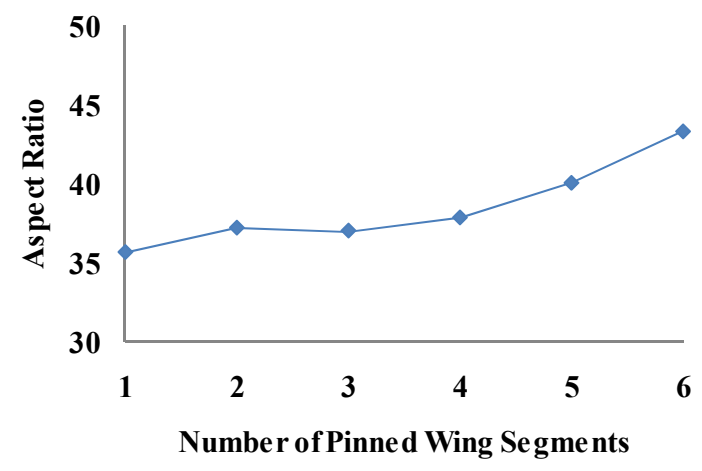

Figure 86 - Optimal Aspect Ratio for Multiple Wing Segments 


\section{CONCLUSION}

The central goal of this research was the development of a multidisciplinary tool for analysis, design, and optimization of HALE UAVs, facilitating the study of a novel configuration concept. Applying design ideas stemming from a unique WWII-era project, a "pinned wing" HALE aircraft would employ self-supporting wing segments assembled into one overall flying wing. The research effort began with the creation of a multidisciplinary analysis environment comprised of analysis modules, each providing information about a specific discipline. As the modules were created, attempts were made to validate and calibrate the processes against known data, culminating in a validation study of the fully integrated MDA environment. Using the NASA / AeroVironment Helios aircraft as a basis for comparison, with generalized Helios payload and mission data, the included MDA environment sized a vehicle to within $5 \%$ of the actual maximum gross weight. Because certain mission specifics for Helios were unclear or non-specific, like battery weight or cruise profile, several sensitivity studies were completed. In addition to showing correct trends and system responses to certain variable perturbations, these studies identified some of the stronger drivers of aircraft weight, like energy storage technology or mission definition (cruise altitude and endurance). With reassurance that the tool provides reasonable results and correct trends, the whole process was wrapped in an optimization environment for further study.

Because of the parametric approach to the problem at hand, comparison of optimum results is focused on evaluating the change from some baseline configuration. First, the Helios-based validation case was re-posed as an optimization problem to see if 
any improvements were possible. Here, improvements may be in the form of reduced gross weight, or enhanced mission capabilities. For Helios technology and a mediumendurance mission (14-15 hours) the MDO tool shows a $17.3 \%$ reduction in gross weight, largely due to a thicker wing for structural weight reduction, and an optimized planform for low power flight, reducing battery weight. Next, more advanced technology parameters were used with the Helios-style aircraft to perform a long-endurance mission with requirements similar to the DARPA Vulture program. With $10 \%$ more efficient solar cells, a $92 \%$ net power train efficiency, and advanced batteries, the aircraft was able to achieve day/night operation at a gross weight nearly identical to the optimized mediumendurance configuration. All subsequent optimizations consider advanced technology components and a long endurance mission, so this last case is used as a baseline for comparison of the segmented wing concept.

When applying the MDO tool to a multi-segment flying wing architecture, we expect that the reduced aspect ratio of each individual wing section should reduce the overall structural weight of the aircraft. Indeed, moving from the baseline to a two segment wing allows a 50\% reduction in wing structural weight in addition to lowered battery and solar weights. Overall, the two segment wing provides an $8.5 \%$ reduction in gross weight over the long-endurance baseline for the same mission and payload requirements. It was shown that increasing the number of wing segments provides diminishing benefit, and that when certain constraints are activated, like the lower bound of $t / c$, some solution trends may change but the resulting gross weight is not greatly affected. For the six segment configuration, the MDO tool predicts a $14.2 \%$ decrease in weight compared to the long endurance baseline. 
Upon conception of this research effort, it was thought that implementing a pinned wing concept may facilitate aircraft of extremely high aspect ratio and great improvement over a one-piece wing. We have shown that in fact the concept does allow for reduced structural weight, low wing loading, and optimal aspect ratios in the high 30s to low 40s. However, with advancements in energy storage technologies, highly efficient propulsion devices, and a careful spanwise mass distribution applied to a one-piece flying wing, many of the same benefits are possible. The added complexity inherent in a pinned wing configuration, and the associated risk detract from the potential benefits. Where we initially expected drastic improvements, results from the MDO tool suggest that actual benefits may not be quite as outstanding. 


\section{FUTURE WORK}

Due to the modular nature of the MDO tool created for this research, one main area for future work is the improvement of the disciplinary analysis codes. Admittedly, the author is not an expert in all areas of flight science, composite structural analysis, aeronautical alternative energy, etc., but the included subroutines are meant to give a good first order approximation of trends and sensitivities. It may be worthwhile to investigate whether replacing the analysis modules with high-fidelity counterparts provides different predictions of parametric changes, or deltas.

Some other possible areas for future work may address some of the supplemental aspects of design that were either not directly addressed in the included study, or were attempted but not completed. First of note is a modeling of the vehicle in a dynamic environment capable of performing gust load analysis or structural dynamic responses. ASWING is a program developed to evaluate structural, aerodynamic, and control response characteristics of aircraft with high aspect ratio wings and highly flexible structures [56]. Currently the MDO tool creates an ASWING configuration input file that includes the aerodynamic layout, structural details, and weight distribution. For a single segment wing, analysis in ASWING is straightforward, but problems were encountered when defining a multi segment platform. We were unable to develop a sufficient and accurate set of kinematic constraints for a pinned wing that satisfied ASWING's requirements.

Also, the tool applies to a wide range of scales of aircraft, so studies of small payload aircraft are possible. Or, if keeping with a large payload, alternate CONOPS may 
lead to modification of optimizer constraints like wingspan and may result in different vehicle solutions. Other configurations may also be investigated, as small changes in the tool allow for aircraft that may include conventional tails and tail booms.

Lastly, the basis of the study discussed in this paper was an aircraft that included chemical batteries as the energy storage medium. However, because the tool implements the energy system sizing methods presented by Dudley and Misra [36], this may be changed to completely alter the aircraft concept. Inclusion of a fuel-cell based power system is simply a matter of changing one input flag, and new propulsive efficiencies are calculated, new components are sized for the weight statement including things like hydrogen tanks, and new energy density values are used. 


\section{REFERENCES}

[1] Hall, D. W., Watson, D. A., Tuttle, R. P., and Hall, S. A., "Mission Analysis of Solar Powered Aircraft," NASA CR-172583, July 1985.

[2] Phillips, W. H., "Some Design Considerations for Solar-Powered Aircraft,” NASA-TP-1675. June, 1980.

[3] Hall, D. W., Fortenbach, C. D., Dimiceli, E. V., and Parks, R. W., "A Preliminary Study of Solar Powered Aircraft and Associated Power Trains,” NASA-CR-3699. Dec, 1983.

[4] Romeo, G., Frulla, G., Cestino, E., and Corsino, G., "HELIPLAT: Design, Aerodynamic, Structural Analysis of Long-Endurance Solar-Powered Stratospheric Platform," AIAA Journal of Aircraft, 2004. Vol. 41, no. 6 (1505-1520).

[5] "Vulture Program: Broad Agency Announcement (BAA) Solicitation 07-15," Defense Advanced Research Projects Agency, DARPA/TTO. Arlington, VA. July, 2007.

[6] Carty, A., "An Approach to Multidisciplinary Design, Analysis and Optimization For Rapid Conceptual Design," $9^{\text {th }}$ AIAA/ISSMO Symposium on Multidisciplinary Analysis and Optimization.. Sept. 2002. AIAA 2002-5438.

[7] Logan, T. R., "Strategy for Multilevel Optimization of Aircraft," AIAA Journal of Aircraft, 1990. Vol. 27, no. 12 (1068-1072).

[8] “2007-2008 AIAA Graduate Team Aircraft Design Competition,” AIAA Student Programs, Reston, VA.

[9] Noth, A., "History of Solar Flight," Anonymous Systems Lab, ETH, Zurich, Switzerland., Jul. 2008.

[10] "History of Electric Flight," URL: http://www.iriquois.free-online.co.uk/hist.htm . [cited 28 Apr 2009].

[11] "Overview of Energy Storage Methods," MORA Associates, URL: http://www.moraassociates.com . [cited 28 Apr 2009].

[12] "Lithium Sulfur Rechargeable Battery Data Sheet," SION Powe Inc., URL: http://www.sionpower.com/pdf/sion_product_spec.pdf . [cited 28 Apr 2009].

[13] Tudron, F. B., Akridge, J. R., and Puglisi, V. J., "Lithium-Sulfur Rechargeable Batteries: Characteristics, State of Development, and Applicability to Powering Portable Electronics," Scion Power Corporation, Tucson AZ, 2004.

[14] Dents, D. J., "Solar Airplanes and Regenerative Fuel Cells," Electrochemistry Branch, NASA Glenn Research Center at Lewis Field, 9 Oct. 2007.

[15] "The History of Solar," U.S. Department of Energy, Energy Efficiency and Renewable Energy, URL: http://www1.eere.energy.gov/solar/pdfs/solar timeline.pdf . [cited 29 Apr 2009].

[16] “AC Propulsion’s Solar Electric Powered Solong UAV,” AC Propulsion, 2 Dec. 2004.

[17] “Solar-Power Research at Dryden,” NASA Facts, Dryden Flight Research Center FS-1998-10-0054 DFRC.

[18] "Pathfinder and the Development of Solar Rechargeable Aircraft," URL: http://www.llnl.gov/etr/pdfs/07 94.1.pdf. [cited 28 Apr 2009].

[19] "NASA Dryden Fact Sheet - Pathfinder Solar-Powered Aircraft," URL: http://www.nasa.gov/centers/dryden/news/FactSheets/FS-034-DFRC.html . [cited 28 Apr 2009].

[20] Noll, T. E., Brown, J. M., Perez-Davis, M. E., Ishmael, S. D., Tiffany, G. C., and Gaier, M., "Investigation of the Helios Prototype Aircraft Mishap," Helios Prototype Aircraft Mishap Investigation Board, 16 Jan 2004. 
[21] "Helios Prototype: The forerunner of $21^{\text {st }}$ century solar-powered 'atmospheric satellites'," NASA Dryden Flight Research Center, URL: http:/www.nasa.gov/centers/dryden/news/FactSheets/FS-068-DFRC.html [cited 1 Feb 2009].

[22] Anderson, C. E., "Dangerous Experiments: Wingtip Coupling at 15,000 Feet," Flight Journal, Vol. 5, No. 6, p. 64 , Dec 2000 .

[23] "Convair GRB-36F Peacemaker," National Museum of the US Air Force, URL: http://www.nationalmuseum.af.mil/factsheets/factsheet.asp?id=2550 . [cited 31 May, 2009].

[24] Miller, J., “Project ‘Tom-Tom',” Aerophile, Vol. 1, No. 3, pp. 161-164, Dec 1977.

[25] Diedrich, F. W., and Zlotnick, M., "Calculated Lift Distributions of a Consolidated Vultee B-36 and Two Boeing B-47 Airplanes Coupled at the Wing Tips," National Advisory Committee for Aeronautics. NACA RM L50I26. Nov 30, 1950.

[26] Jensen, S.C., Rettie, I.H., and Barber, E.A., "Role of Figures of Merit in Design Optimization and Technology Assessment," Journal of Aircraft, Vol. 18, No. 2, Feb 1981.

[27] "Cost and Business Model Analysis for Civilian UAV Missions,” Moiré Incorporated, June 8, 2004.

[28] "Constrained Optimization," The MathWorks, Inc., Optimization Toolbox ${ }^{\mathrm{TM}}$, MATLAB Documentation, 1984-2008.

[29] Fletcher, R., Practical Methods of Optimization, Second Edition, John Wiley \& Sons Ltd, West Sussex, England, 1987.

[30] Kroo, I, "Distributed Multidisciplinary Design and Collaborative Optimization," VKI lecture series on Optimization Methods \& Tools for Multicriteria/Multidisciplinary Design, Stanford University, Nov. 2004.

[31] Sobiesxcxanski-Sobieski, J., "A Linear Decomposition Method for Large Optimization Problems-Blueprint for Development," NASA TM-83248, Feb.1982.

[32] Steward, D., "The Design Structure System: A Method for Managing the Design of Complex Systems," IEE Transactions of Engineering Management, Vol. EM 78. No. 3, Aug. 1981.

[33] Hall, D. W., and Hall, S. A., "Structural Sizing of a Solar Powered Aircraft," NASA CR-172313, April, 1984.

[34] Frulla, G., and Cestino, E., "Design, manufacturing, and testing of a HALE-UAV structural demonstrator," Composite Structures, April 2008. Vol. 83, issue 2, pp 143-153. ISSN 0263-8223.

[35] Nam, T., "A Generalized Sizing Method for Revolutionary Concepts Under Probabilistic Design Constraints," Ph.D. Thesis, School of Aerospace Engineering, Georgia Institute of Technology, May 2007.

[36] Dudley, M., and Misra, A., "Electric Airplane Power-system Performance Requirements," The 2009 CAFE Foundation Electric Aircraft Symposium. NASA Ames and Glen Research Centers. 24 Apr. 2009.

[37] Youngblood, J. W., Talay, T. A., and Pegg, R. J., "Design of Long-Endurance Unmanned Airplanes Incorporating Solar and Fuel Cell Propulsions,” AIAA Paper 84-1430, June 1984.

[38] Drela, M., and Youngren, H., "AVL: Aerodynamic Analysis, Trim Calculation, Dynamic Stability Analysis, Aircraft Configuration Development," Massachusetts Institute of Technology, URL: http://web.mit.edu/drela/Public/web/avl// . [cited 20 May, 2009].

[39] "Empirical Drag Estimation Technique (EDET) Program Users Manual," Lockheed California Company, Burbank, CA. LR 28788. 22, Dec 1978. 
[40] Feagin, R. A. and Morrison, W. D., Jr., "Delta Method, and Empirical Drag Buildup Technique,” NASA CR 151971, Dec 1978.

[41] "Steve Fossett: Always 'Scouting for New Adventures' Part 2," Airport Journals, URL: http://www.airportjournals.com/Display.cfm?varID=0611030, Nov 2006. [cited 25 May, 2009].

[42] "Non-Stop Solo Around the World," American Aviation Historical Society, URL: http://www.aahsonline.org/articles/globalflyer.htm . [cited 25 May, 2009].

[43] Duffie, J. A., and Beckman, W. A., Solar Engineering of Thermal Processes, Wiley-Interscience, New York, third edition, 2006.

[44] Noth, A., "Design of Solar Powered Airplanes for Continuous Flight," Dissertation, Doctor of Technical Sciences, ETH Zurich. Diss. ETH No. 18010. Sept, 2008.

[45] "Solar Impulse Version 1.1 Electrical Values Presentation," Ecole Polytechnique Federale De Lausanne, Apr. 2004.

[46] Raffaelle, R. R., Harris, J. D., Hehemann, D., Scheiman, D., Rybicki, G., and Hepp, A. F., "Integrated ThinFilm Solar Power System," Energy Conversion Engineering Conference and Exhibit, 2000. (IECEC) 35th Intersociety, vol.1, no., pp.58-62 vol.1, 2000.

[47] Cestino, E., "Design of Solar High Altitude Long Endurance Aircraft for Multi Payload \& Operations," Department of Aerospace Engineering, Politecnico di Torino, Turin, Italy. June 2006.

[48] Colozza, A. J., "Effect of Power System Technology and Mission Requirements on High Altitude Long Endurance Aircraft," NASA CR-194455. Nov. 1993.

[49] Nickol, C. N., Guynn, M. D., Kohout, L. L., and Ozoroski, T. A., "High Altitude Long Endurance UAV Analysis of Alternatives and Technology Requirements Development," NASA/TP-2007-214861. Mar. 2007.

[50] “Our Tax Dollars at Work,” URL: http://www.stango.net/helios.htm .[cited 25 May, 2009].

[51] Center for Composite Material Research, North Carolina A\&T State University, http://www.ncat.edu/ ccmradm/ccmr/ [cited 20 Apr. 2009].

[52] Quantum Composites Lytex® 4149 55\% Carbon Fiber Epoxy SMC Data Sheet. MatWeb Material Property Data. $\quad$ http://www.matweb.com/search/DataSheet.aspx?MatGUID=3b8b3df085d2448fad7436fa7c492107 [cited 1 May 2009].

[53] Wainfan, B., “Aerodynamics of Long Endurance Flight,” ACA Industries, Inc., Cal Poly Aircraft Design Lab Library XA 500.

[54] Baraaclough, J., "Electric Propulsion Platforms at DFRC,” NASA DFRC-943. 10, Apr. 2009.

[55] Chan, C. K., Peng, H., Liu, G., McIlwrath, K., Zhang, X. F., Huggins, R A., Cui, Y., "High-performance Lithium Battery Anodes Using Silicon Nanowires,” Nature Nanotechnology. 16 Dec. 2007.

[56] Drela, M., "ASWING Configuration Development System for Flexible Aircraft," Massachusetts Institute of Technology, URL: http://web.mit.edu/drela/Public/web/aswing/ . [cited 29 May, 2009]. 